\title{
MASTEA
}

\section{Assessment of Contemporary Mathematical Methods for Magnetic Fusion Research}

March 1978

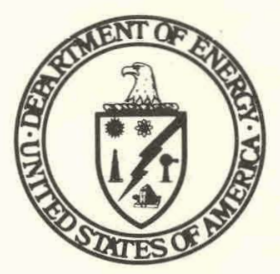

Prepared for

U.S. Department of Energy

Assistant Secretary for Energy Technology

Office of Fusion Energy

Under Contract No. EA-77-X-01-2865 


\section{DISCLAIMER}

This report was prepared as an account of work sponsored by an agency of the United States Government. Neither the United States Government nor any agency Thereof, nor any of their employees, makes any warranty, express or implied, or assumes any legal liability or responsibility for the accuracy, completeness, or usefulness of any information, apparatus, product, or process disclosed, or represents that its use would not infringe privately owned rights. Reference herein to any specific commercial product, process, or service by trade name, trademark, manufacturer, or otherwise does not necessarily constitute or imply its endorsement, recommendation, or favoring by the United States Government or any agency thereof. The views and opinions of authors expressed herein do not necessarily state or reflect those of the United States Government or any agency thereof. 


\section{DISCLAIMER}

Portions of this document may be illegible in electronic image products. Images are produced from the best available original document. 


\section{NOTICE}

This report was prepared by Yvain M. Treve as an account of work sponsored by the United States Government. Neither the United States nor the United States Department of Energy, nor any of their employees, nor any of their contractors, subcontractors, nor any of their employees, makes any warranty, express or implied, or assumes any legal liability or responsibility for the accuracy, completeness, or usefulness of any information, apparatus, product or process disclosed, or represents that its use would not infringe privately owned rights. 
Errata: HCP/T2865-01

UC-20g

ASSESSMENT OF CONTEMPORARY MATHEMATICAL METHODS FOR MAGNETIC FUSION RESEARCH

AUTHOR: Yvain M. Treve

The attached title page has been inadvertently omitted from the subject document . 
HCP/T2865-01

$U C-20 g$

\title{
ASSESSMENT OF CONTEMPORARY MATHEMATICAL METHODS FOR MAGNETIC FUSION RESEARCH
}

\author{
March 1978 \\ Yvain M. Treve \\ Prepared For \\ U.S. DEPARTMENT OF ENERGY \\ Assistant Secretary for Energy Technology \\ Office of Fusion Energy \\ Washington, D.C. 20545 \\ Under Contract No. EA-77-X-01-2865
}


This report was prepared is an account of work sponsored by the United States Covernment. Neither the

United States nor the United States Department of

Energy, nor any of their employees, nor any of their

contractor, subcontractors, of their employees, makes

any warranty, express or inplied, or assumes any legal liability or responsibility for the accuracy, completenes

or usefultess of any information, apparatus, product or

process disclosed, or represents that its use would no

infringe privately owned rights.

$$
-\cdot
$$




\section{NOTICE}

Ihis report was prepared as an account of work sponsored by the United States Govcrnment, Neither the United Statoo nor the United States Department of Energy, nor any of their employees, makes any warranty, express or implied, or assumes any legal liability or responsibility for the accuracy, completeness, or usefulness of any information, apparatus, product, or process disclosed, or represents that its use would not Infringe privately owned rights, Reference herein to any specific commercial product, process, or service by trade name, mark, manufacturer, or otherwise, does not necessarily constitute or imply its endorsement, recommendation, or favoring by the United States Government or any agency thereof. The vilews and opinions of authors expressed herein do not necessarily state or reflect those of the Untted States Government or any agency thereof.

Available from:

National Technical Information Service (NTIS) U.S. Department of Commerce 5285 Port Royal Road Springfield, Virginia 22161

Price: $\quad$ Printed Copy: $\quad \$ 6.50$

Microfiche: $\$ 3.00$




\section{FOREWORD}

This work was commissioned by the U.S. Department of Energy in order to bring to the attention of plasma theorists some useful advances in applied mathematics. Y. M. Treve, who over the years contributed to the solution of many nonlinear problems of practical interests (shock waves, energy conversion, plasma physics, as well as upper atmospheric and magnetospheric physics), has concentrated principally on four topics overlapping some areas of current and near-term interest to fusion plasma theory: (a) the Kolmogorov-Arnold-Moser theorem and its applications; (b) the use of Smale's horseshoe and symbolic dynamics for describing stochasticity in nonlinear systems with a few degrees of freedom; (c) Hopf bifurcation theorem and its application to the study of stability of nonlinear systems; (d) strange attractors, esp. the Lorenz attractor -a mathematical concept gaining some currency in contemporary models of turbulence.

The motivation leading to this review was to emphasize in the context of plasma physics the physical content of the mathematical results, rather than presentation of the rigorous results. The selection of topics was governed by the desire to enlarge the "toolkit" of those interested in attaching nonlinear problems in plasma physics, rather than sharpen or improve existing methods and approaches to these problems.

Comments and inquiries may be addressed to the author at 125 San Rafael Avenue, Santa Barbara, California, 93109.

Oscar P. Manley

Fusion Theory Branch

Applied Plasma Physics Program

Office of Fusion Energy 
TABLE OF CONTENTS

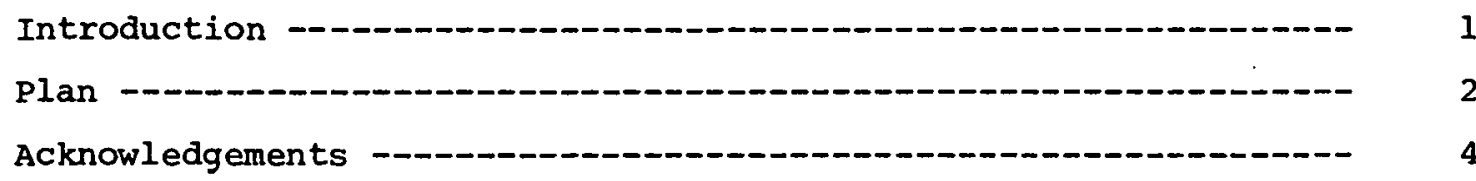

1. Chaotic Motions in Hamiltonian Systems

1.1 Earlier theory of Hamiltonian Systems -

1.2 The Kolmogorov-Arnold-Moser Theorem - 8

1.3 Poincare Maps and Area-preserving Mappings --------- 19

1.4 Stability in the Large of Hamiltonian Systems -- 43

1.5 Non-canonical Perturbations of Hamiltonian Systems ---- 43

1.6 Method of Averaging - 45

2. Chaotic Motions in Dissipative Systems: The Case of strong Turbulence - - 50

2.1 Hopf Bifurcation Phenomenon - 64

2.2 The Ruelle-Takens Picture of Turbulence - - 66

2.3 The Lorenz Model - 69

Appendix A: Superconvergent Perturbation Method - 79

Appendix B: Note on the KAM Theorem 83

Appendix C: Periodic Solutions of a slightly Perturbed Integrable Hamiltonian System by the PCHG Method - 84

Appendix D: Cantor Sets - 90

Source -

References - $-1-0-106$ 
The mathematical techniques that we review in this report have been selected on the basis of their relevance to at least four outstanding theoretical problems of magnetic fusion research, namely: a) ion heating; b) particle-wave interactions; c) stability of magnetic surfaces in real tokamaks; and d) strong plasma turbulence. These problems have a common feature: they all involve chaotic motions in spite of the perfectly deterministic nature of the mathematical models used for their description.

The status of our understanding about these chaotic motions differs markedly depending on the type of the mathematical models:

Regarding problems a), b); and c), their models belong to the class of the so-called non-integrable Hamiltonian systems which always possess some solutions with a chaotic behavior. To be exact, the analytical proof of this property has been given only for systems with two degrees of freedom but there is ample evidence from several theoretical and numerical computations that the property belongs generically to every non-integrable Hamiltonian system.

As to turbulence, even in hydrodynamics, there is no rigorous proof available that the Navier-stokes equations possess turbulent solutions. This is an extremely difficult problem because the phase-space of these equations, i.e. the space of all possible velocity vector fields, has an infinite number of dimensions. Physicists and mathematicians have invoked in the past various mathematical phenomena in order to explain how the Navier-stokes equations can possess turbulent solutions but none of these early conjectures has survived. Even the famous picture proposed by Landau in the early forties is no longer tenable since several of its implications are contradicted by experiment. 
Recently, however, a new picture has been proposed by the mathematicians Ruelle and Takens which does not suffer from the defects of Landau's picture and is mathematically more plausible.

The novel and central element of the Ruelle-Takens picture is a completely new kind of mathematical objects called strange attractors. In contrast with the well known attractors such as stable nodes, foci, or limit cycles, a strange attractor "is a highly complex structure sitting in the phase-space of the dynamical equations which traps for ever all orbits passing nearby. Its most important property is that it imparts to these motions a chaotic character and an extreme sensitivity to the initial conditions.

Although the Ruelle-Takens picture is no more than a guess at the present time it is generally believed that the actual state of affairs is not too far from it. This belief is based on preliminary mathematical results which suggest that most equations like the Navier-Stokes equations possess strange attractors in their phase-space. Also, as already mentioned, the picture does not have the defects of Landau's picture and is in qualitative agreement with several experiments specially designed to test its validity.

The important point that we want to make in this report is this: on the basis of already proven facts there is strong evidence that most realistic mathematical models of complex physical phenomena possess under certain conditons some solutions with a random, chaotic behavior. It must be emphasized that the chaotic character of these snlutinns is intrinsis to the model and does not require any additional assumptions about the occurence of randomness. Moreover, the set of initial conditions corresponding to these solutions has finite measure, i.e. they are observable. It appears therefore indispensable that physicists be aware of the possible existence of such solutions in order not to miss new ways of achieving some desirable 
effects (e.g. ion heating by electrostatic waves) and correctly interpret some experimental data. Finally; we feel that every physicist's panoply of mathematical concepts should include that of strange attractor as a new possible model of disorder in dissipative phenomena.

The kind of mathematics necessary nowadays for the in-depth study of dynamical systems is unknown to most physicists: abstract algebra, general topology, differential topology, algebraic topology, global analysis, analysis on manifolds, etc. We hope however that we have managed to write our expose in a form readily understandable to the reader not versed in these disciplines. Plan

The main text is divided into two sections.

In the first section devoted to Hamiltonian systems we briefly review the essentials of the Hamilton-Jacobi theory and discuss the Kolmogorov-Arnold-Moser theorem and its implications. This gives us the opportunity to expose various standard mathematical notions and methods. In particular we present the most recent developments in pexturbation theory, notably the so-called superconvergent schemes. We also discuss at some length Poincare's method of section and areapreserving mappings for two-degree of freedom systems. In this connection, Smale's horseshoe map and its properties are described to give an idea of the methods used in symbolic dynamics for the study of random motions. This is followed by an examination of the phenomena assoriated with an increase in the size of the perturbation and a review of the various avenues presently being followed for the determination of the onset of stochasticity. We suggest that the modern version of an old method of Poincare, the PCHG method (for PoincareCesari-Hale-Gambill), can perhaps be used to elucidate some aspects of this problem and we indicate how to apply it to a wide class of slightly perturbed integrable systems. 
The rest of the section is devoted to a review of same recent mathematical results. which seem of potential.value for plasma physics and to a discussion of the method of averaging and its limitations.

In section 2 we review the difficulties of the problem of turbulence and present the Ruelle-Takens picture. An example of a dynamical system with a strange attractor is constructed and the Hopf bifurcation theory is discussed. Finally we review the properties of the Lorenz model for the convective instability of an atmospheric layer which is known to have a strange attractor for sufficiently high Rayleiqh numbers.

Some of the more mathematical derivations are relegated to several appendices. References indicated by an $S$ followed by a number are listed at the end under the heading "Sources". We give there selected sources for further reading as well as various remarks and comments.

\section{Acknowledgements}

First, I should like to express my profound gratitude to Dr. Oscar Manley for his constant support and guidance during the preparation of this report. I have also benefited greatly from conversation with Professors S. Smale, J.K. Hale, J. LaSalle, J.A. Yorke, A. Weinstein, R.B, Leipnik and A.J. Dragt. I am particularly indebted to Professor Alan Kaufman for having read the manuscript; his constructive criticisms have been invaluable to me. Furthermore, I should like to express my appreciation to Mrs. Sarah Wilson for having typed the manuscript.

1. Chaotic Motions in. Hamiltonian Systems

1.1 Earlier Theory of Hamiltonian Systems

We recall that Hamiltonian systems are of the form (s-1)

$$
\dot{q}=\frac{\partial H}{\partial p}, \dot{p}=-\frac{\partial H}{\partial q}
$$

where $q, p$ are n-vectors $q=\left(q_{1}, \ldots, q_{n}\right), p=\left(p_{1}, \ldots, p_{n}\right)$, the dot denotes differentiation with respect to the time $t$, and $H$, the Hamiltonian, is a given function of these variables which may also depend upon the time. The components 
of $q$ and $p$ are called the generalized coordinates and momenta, respectively. Each pair $\left(q_{i}, p_{i}\right)$ is associated: with one of the $n$ degrees of freedom of the system.

It is always possible to convert a time-dependent Hamiltonian into a time-independent one by adding one more degree of freedom. We shall take advantage of this possibility to restrict ourselves to time-independent Hamiltonians $H(q, p)$. Then $H$ is the total energy $E$ of the system which is a constant of the motion, i.e. $H(q, p)$ is a first integral of Eqs (1) which can be used to reduce their order by one.

of special importance are those Hamiltonian systems for which $\mathrm{n}$ singlevalued independent first integrals $F(q, p)$ are known and are such that $\left[F_{i}, F_{j}\right]=0$ for all $i, j=1,2,3, \ldots, n$ where the poisson bracket of two arbitrary functions of $q$ and $p$ is defined by

$$
[U, v]=\sum_{i=1}^{n}\left(\frac{\partial U}{\partial q_{i}} \frac{\partial V}{\partial p_{i}}-\frac{\partial U}{\partial p_{i}} \frac{\partial V}{\partial q_{i}}\right)
$$

First integrals satisfying this condition are said to be in involution. In general, knowledge of a first integral of a differential system allows a reduction of its order by one. For Hamiltonian systems we can do better: with a known first integral different from the Hamiltonian we can reduce the order of Eqs (1) by 2 and if we know $n$ integrals in involution we can completely solve the problem according to the following procedure:

We seek a change of variables $q, p \rightarrow q^{\prime}, p^{\prime}$ such that the differential equations for the new variables are derivable from a new Hamiltonian $H^{\prime}\left(q^{\prime}, p^{\prime}\right)$ as the Eqs (1) for the old ones are derived from $H(q, p)$, i.e.

$$
\dot{q}^{\prime}=\frac{\partial H^{\prime}}{\partial p^{\prime}}, \dot{p}^{\prime}=-\frac{\partial H^{\prime}}{\partial q^{\prime}} \text {. }
$$

A transformation possessing this property is called canonical and from the Hamilton-Tacobi theory is derivable from a characteristic function $S\left(q, \underline{p}^{\prime}\right)$ according to the prescription

$$
q^{\prime}=\frac{\partial S}{\partial p^{\prime}}, p=\frac{\partial S}{\partial q}
$$


The importance of knowing a set of $n$ single-valued first integral in involution is due to the fact that it is then possible to construct a characteristic function $S$ such that the new Hamiltonian does not depend upon $q^{\prime}: H^{\prime}\left(q^{\prime}, p^{\prime}\right)=H^{\prime}\left(p^{\prime}\right)$. Indeed this implies by Eq $(2 \mathrm{~b})$ that $\dot{\mathrm{p}}^{\prime}=0$, hence $\mathrm{p}^{\prime}$ and therefore $\partial \mathrm{H}^{\prime} / \partial \mathrm{p}^{\prime}=$ $\dot{q}^{\prime} \equiv \omega$ are constant $\mathrm{n}$-vectors so that Eqs (2) are readily integrated:

$$
q^{\prime}(t)=\omega t+q_{0}^{\prime}, p^{\prime}=p_{0}^{\prime}, \quad(4 a, b)
$$

and $q(t)$ and $p(t)$ are obtained via the inverse transformation. The characteristic function which allows us to achieve this feat is found thus: set the $n$ integrals $F_{i}$ (or any set of $\mathrm{n}$ functionally independent combinations of them) equal to the components of the new momenta, i.e.

$$
F(q, p)=p^{\prime}
$$

and solve this vector equation for $p=p^{*}\left(q, p^{\prime}\right)$. The differential form $p *\left(q, p^{\prime}\right) . d q$ is then the perfect differential of $s$ which is therefore given by

$$
s\left(q, p^{\prime}\right)=\int^{q} p^{*}\left(q, p^{\prime}\right) \cdot d q
$$

Once $S$ is computed Eqs (3) are solved for $q=q^{*}\left(q^{\prime}, p^{\prime}\right)$; substitution of this into $(3 b)$ gives $p=p *\left(q^{\prime}, p^{\prime}\right)$ and the new Hamiltonian automatically does not depend upon $\mathrm{q}^{\prime}$. The solution of Eqs (1) is then obtained as

$$
q(t)=q^{*}\left(\omega t+\frac{\partial S}{\partial p}, \quad F\left(q_{0}, p_{0}\right)\right), p(t)=\frac{\partial S}{\partial q}\left(q(t), F\left(q_{0}, p_{0}\right)\right)
$$

where, in $\partial S / \partial p^{\prime}, p^{\prime}=F\left(q_{0}, p_{0}\right)$ and $q=q_{0}$.

Hamiltonians for which $\mathrm{n}$ integrals in involution are known axe said to be integrable. But no systematic method is known for finding first integrals and there is to-date no analytical test to decide whether a given Hamiltonian system has single valued first integrals besides the Hamiltonian. The only approach available is via exploration of the geometrical configuration of the phase-space by numerical integration of the orbits. 
It was recognized long ago that for the many integrable problems of classical mechanics the manifold $M$ defined by $F_{i}(q, p)=$ constant, $i=1,2, \ldots, n$, is an n-dimensional torus, i.e. the topological product of $n$ circumferences. Arnold (s-2) has shown rigorously using topological arguments that it is so whenever at every point of $M$ the gradients of the functions $F$ are linearly independent. On a particular torus corresponding to a given value of $F(q, p)$ the motion is quasi-periodic in general (periodic if the frequencies are commensurable) and given by Eqs (4a) where the coordinates are defined modulo a fundamental period which is usually chosen equal to $2 \pi$. With this choice of the period, the "momenta" have the dimension of action and the coordinates are angles $(\mathrm{S}-3)$.

It is seen from Eqs (4a) that depending upon the values of the frequencies $\omega_{i}$ and therefore on the torus the motion may be periodic or quasiperiodic (some authors say multiply periodic; the old equivalent expression "conditionally periudic" is progressively being abandoned). It is periodic for those values of $q_{0}$ and $p_{0}$ such that there is a time $T$ for which $\omega_{i} T=$ $k_{i} 2 \pi, k_{i}$ an integer, $i=1,2, \ldots, n, i . e$. when the frequencies are commensurable; then the orbits are closed curves on the torus. If instead all frequencies are incommensurable for a set of values of $q_{0}, p_{0}$ then the orbits fill the corresponding torus like a Lissajous curve. The set of such tori has positive measure while the set of tori on which the motion is periodic has zero measure since it is in one-to-one correspondence with the rational numbers.

We thus see that the behavior of integrable Hamiltonian systems is fairly well understood. In sharp contrast, the behavior of systems for which n sinqle-valued integrals in involution do not exist is extremely complicated. Poincare was the first to realize this fact in connection with his investigation of the restricted problem of 3 bodies. Taking advantage of the possibility of 
reducing this problem to a Hamiltonian system with only two degrees. of freedom he obtained many important results some of which are generic, i.e. are to be found for all Hamiltonian systems of this kind (13). Following the path opened by Poincare, Birkhoff (14) found many important properties of systems with two degrees of freedom which shed some light on the complexity of their behavior. But it is only recently that a full measure of this complexity and of its possible implications in mechanics and physics was achieved through both analytical and numerical work.

\subsection{The Kolmogorov-Arnold-Moser. Theorem}

A major landmark in the evolution of our knowledge about non integrable systems was the announcement by Kolmogorov in 1954 of his famous theorem on the behavior of slightly perturbed Hamiltonian systems (cf ref. 7, Appendix 7). Kolmogorov gave only a sketch of the proof (15) which was later carried out in details by Arnold (16), (s-4) for analytic Hamiltonians. Finally, Moser $(s-5)$ gave another proof under weaker assumptions. The theorem has thus become to be known as the KAM theorem for short.

A good understanding of the KAM theorem requires some familiarity with the theory of perturbations developed by the astrnnomers for the computation of the orbits of heavenly bodies. The theory deals with Hamiltonians of the general form

$$
H(q, p)=H_{0}(q, p)+\varepsilon H_{1}(q, p, \varepsilon)
$$

where $\varepsilon$ is a small parameter and the Hamiltonian system associated with the unperturbed Hamiltonian $\mathrm{H}_{\mathrm{O}}$ is integrable in the sense indicated before. We may therefore assume that the appropriate canonical transformation has been applied to the original system so that $H_{0}$ depends upon $p$ only. We further assume that $\mathrm{H}_{1}$ is analytic in $\varepsilon$ and can thereforc write the Hamiltonian in the form 


$$
H(q, p)=H_{0}^{H}(p)+\varepsilon H_{1}(q, p)+\varepsilon^{2} H_{2}(q, p)+\ldots \cdot
$$

Many different schemes are available (S-6) for generating approximations to the solution and they are all more or less equivalent differing only in their relative ease of implementation. We choose to present the scheme indicated by Arnold (12). This scheme is distinct from the superconvergent scheme suggested by Kolmogorov and developed by Arnold for the proof of the theorem (see ref. 16 and Appendix A).

Arnold seeks a transformation $q, p \rightarrow q^{\prime}, p^{\prime}$ defined by a characteristic function $\mathbf{S}$

$$
q^{\prime}=q+\varepsilon \frac{\partial S}{\partial p^{\prime}}, \quad p=p^{\prime}+\varepsilon \frac{\partial S}{\partial q}
$$

such that in the new Hamiltonian the terms depending on $q^{\prime}$ be of order $\varepsilon^{n}$ with $n \geq 2$, i.e.

$$
\mathrm{H}(\mathrm{q}, \mathrm{p})=\mathrm{H}_{\mathrm{o}}^{\prime}\left(\mathrm{p}^{\prime}, \varepsilon\right)+\varepsilon^{2} \mathrm{H}_{1}^{\prime}\left(\mathrm{q}^{\prime}, \mathrm{p}^{\prime}\right)+\varepsilon^{3} \ldots
$$

If this can be accomplished, during a time interval $t \simeq 1 / \varepsilon$, the actual solution $q^{\prime}(t), p^{\prime}(t)$ will differ from the motion described by $H_{0}^{\prime}\left(p^{\prime}, \varepsilon\right)$ by a quantity of order $\varepsilon^{2} t$. Going back to the original $q$ and $p$ this will give approximate expressions for $q(t), p(t)$ with errors $O(\varepsilon)$ over such a time interval. If we continue this process a transformation $q^{\prime}, p^{\prime} \rightarrow q^{\prime \prime}, p^{\prime \prime}$ will bring the Hamiltonian to the form

$$
H(q, p)=H_{O}^{n}\left(p^{\prime \prime}, \varepsilon\right)+\varepsilon^{3} H_{1}^{\prime \prime}\left(p^{\prime \prime}, p^{\prime \prime}\right)+\ldots
$$

and the error will now be of the order of $\varepsilon^{3} t$ over a time interval $t$, and so on. To carry out this program we replace $\mathrm{p}$ in $\mathrm{Eq}$ (5) by the right hand side of Eq (6b) and Taylor expand $H_{0}$ about $p=p^{\prime}$; we thus obtain

$$
H(q, p)=H_{0}\left(p^{\prime}\right)+\varepsilon \frac{\partial H_{o}}{\partial p} \cdot \frac{\partial S}{\partial q}+\varepsilon H_{I}\left(q, p^{\prime}\right)+O\left(\varepsilon^{2}\right) .
$$

We can now take advantage of the $2 \pi$-periodicity of $\mathrm{H}_{1}$ in the $\mathrm{q}$ 's to rewrite this as 


$$
\mathrm{H}(\mathrm{q}, \mathrm{p})=\mathrm{H}_{\mathrm{O}}\left(\mathrm{p}^{\prime}\right)+\overline{\mathrm{H}}_{1}\left(\mathrm{p}^{\prime}\right)+\varepsilon\left(\frac{\partial \mathrm{H}_{\mathrm{o}}}{\partial \mathrm{p}^{\prime}} \cdot \frac{\partial \mathrm{S}}{\partial \mathrm{q}}+\overline{\mathrm{H}}_{1}\left(\mathrm{q}, \mathrm{p}^{\prime}\right)\right)+0\left(\varepsilon^{2}\right)
$$

where $\overline{H_{1}}\left(p^{\prime}\right)$ is the mean value of $H_{1}$ and $\widetilde{H}_{1}\left(q^{\prime}, p^{\prime}\right)=H_{1}\left(p^{\prime}\right)-\bar{H}_{1}\left(p^{\prime}\right)$ has thereforc mean value zero. Clearly Eq (7) will be obtained if we choose S such that

$$
\frac{\partial H_{0}}{\partial p^{\prime}} \cdot \frac{\partial S}{\partial q}+\widetilde{H}_{1}\left(q, p^{\prime}\right)=0
$$

Writing the Fourier expansions of $\widetilde{\mathrm{H}_{1}}$ and $\mathrm{S}$ as

and

$$
\widetilde{H_{1}}\left(q, p^{\prime}\right)=\sum_{k \neq 0} h_{k}\left(p^{\prime}\right) \exp (i(k, q))
$$

$$
S\left(q, p^{\prime}\right)=\sum_{k \neq u} s_{k} \exp (i(k, q)) \text {, }
$$

respectively $\left((k, q)=\sum_{i=1}^{n} k_{i} q_{i}\right)$, and substituting into Eq. (8) yiclds the Fourier coefficients for $s$

$$
S_{k}\left(p^{\prime}\right)=i \frac{h_{k}\left(p^{\prime}\right)}{(k, \omega)}
$$

This is where we encounter the old problem of the sma11. divisors $(k, \omega)=\sum_{i=1}^{n} k_{i} \omega_{i}(12)$ : in order to be able to compute the coefficients for all k's $(k, w)$ must be different from zero which implies that the values of the trequencies must be incommensurable, But even if the w's are incommensurable the procedure will not work in general because as we go to higher and higher harmonics, that is as the modulus of $k$ increases indefinilely, some of the divisors $(k, w)$ will become arbitrarily close to zero. The convergence of the series for $S$ is then in doubt.

This difficulty is encountered in all traditional perturbation methods. Yet their inventors, the astronomers, have used them with great success for the calculation of orhits over very long times. The reasun fui their success is that in practice the frequencies are often such that the divisors become small only for very large values of $|k|$. The corresponding values of the Fourier coefficients are then very small so that by truncating 
the series appropriately an excellent approximation to the solution is obtained. This will become clearer after we have discussed the KAM theorem which can be stated as follows. (see Appendix.B):

$$
\text { Suppose again that } \mathrm{H}(\mathrm{q}, \mathrm{p})=\mathrm{H}_{\mathrm{O}}(\mathrm{p})+\varepsilon_{\mathrm{H}}(\mathrm{q}, \mathrm{p}) \text { is analytic in some }
$$
domain $G$ of the phase-space with $\varepsilon$ a small parameter and $H_{1} 2 \pi$-periodic in the 'q's. If in G either

$$
\Delta_{1}=\operatorname{det}\left(\frac{\partial^{2} \mathrm{H}_{0}}{\partial \mathrm{p}_{i}{ }^{\partial \mathrm{p}_{j}}}\right) \nexists \text { or } \Delta_{2}=\operatorname{det}\left(\begin{array}{ll}
\frac{\partial^{2} \mathrm{H}_{0}}{\partial \mathrm{p}_{i} \partial \mathrm{p}_{j}} & \frac{\partial \mathrm{o}}{\partial \mathrm{p}_{i}} \\
\frac{\partial \mathrm{H}_{0}}{\partial \mathrm{p}_{j}} & 0
\end{array}\right) \nexists 0 \text {, }
$$

then $G$ can be decomposed into two disjoint subsets: $G=G_{1}+G_{2}$, where $G_{1}$ is invariant (i.e. the solution point $q(t), p(t)$ belongs to $G_{1}$ for all times for any initial point $q(0), p(0)$ belonging to $G_{1}$ ) and $G_{2}$ is small compared to $G$ and tends to zero with $\varepsilon$. Moreover $G_{1}$ is composed of invariant $n$-dimensional analytical tori on which the motion is quasi-periodic with frequencies $\omega_{i}=$ $\partial \mathrm{H}_{\mathrm{o}} / \partial \mathrm{p}_{i}$

In other words, most of the invariant tori corresponding to the unperturbed Hamiltonian still exist under a small perturbation. They fill a domain $G_{1}$ which occupies most of phase-space.

Note that the theorem does not tell what happens in the small domain $G_{2}$. We will come back to this question later but first we want to show that the theorem is readily applicable to a problem related to ion heating, namely the non-relativistic motion of an ion in a uniform magnetic field $B=B_{0} \hat{z}$ under the perturbation of an electrostatic wave.

In rectangular soordinates the Hamiltonian can be written in the form $(s-7)$ 


$$
H=\frac{1}{2 m}(\underline{p}+m \Omega y \hat{x})^{2}+e_{0} \sin :(\underline{k} \cdot \underline{r}-\omega t)
$$

Without loss of generality $\underline{k}$ can be chosen parallel to the $y, z$ - plane $\left(\underline{k}=k_{z} \hat{z}+k_{1} \hat{y}\right)$. There is then a time dependent canonical transformation which brings the Hamiltonian to the time independent and dimensionless form (25)

$$
H\left(J_{1}, J_{2}, w_{1}, w_{2}\right)=H_{0}\left(J_{1}, J_{2}\right)+\varepsilon H_{1}\left(J_{1}, w_{1}, w_{2}\right)
$$

where

$$
\mathrm{H}_{0}=J_{1}+\nu J_{2}+\frac{1}{2} B^{2} J_{2}^{2}
$$

and

$$
\mathrm{H}_{1}=\sin \left(\alpha\left(2 \mathrm{~J}_{1}\right)^{\frac{b}{2}} \sin \mathrm{w}_{1}+\mathrm{w}_{2}\right)
$$

Here $J_{1}$ and $J_{2}$ are actions conjugate to the angles $w_{1}$ and $w_{2}, \alpha$ and $\beta$ are the direction cosines of $k, \nu=\omega / \Omega$, and $\varepsilon$ measures the amplitude of the wave. For $\varepsilon=0$, the motion takes place on invariant tori and is periodic or quasi-periodic depending on whether the frequencies $\omega_{1}=1$ and $\omega_{2}=\nu+\beta^{2} J_{2}$ are commensurable or not. The KAM theorem applies for $\varepsilon$ small and in the general case $\beta=k_{z} / k \neq 0$ since then the determinant $\Delta_{2}=\beta^{2} \neq 0$. Hence for $\varepsilon$ small and $\beta \neq 0$ most of the motions take place on invariant tori and are quasi-periodio.

Note that the theorem does not apply in the situation considered by Karney and Bers (22) and Karney (23) where the wave propagates at right angle to the magnetic field since then $\beta=0$ so that $\Delta_{1}=\Delta_{2} \equiv 0$.

For $\varepsilon$ different from zero there are motions other than the quasiperiodic ones which fill a subset of phase-space of small measure and whose behavior, as we shall see, is of an entirely different nature. But first we want to make an important remark: for 2-degree of freedom Hamiltonian systems such as the one above, the phase-space is 4-dimensional but can be 
reduced to 3 dimensions by eliminating one of the variables using the energy integral. This considerably simplifies the study of the orbits especially if we use the ingenious method of sections invented by Poincaré (13; yol. 3 ). Suppose $\gamma$ is a closed periodic orbit and let $\alpha$ be a point on it. Through $\alpha$ let us pass a smooth surface $\sum$ whose tangent plane makes a finite angle with the tangent to $\gamma$ at $\alpha\left(\gamma\right.$ and $\sum$ are then said to intersect transversally). Now pick another point $\beta$ in $\sum$ and close to $\alpha$, and consider the orbit $\gamma^{\prime}$ going through it. By reason of continuity we can expect that after a certain time $\gamma^{\prime}$ will pierce again $\sum$ at a point $\beta_{1}$. If $\beta_{1}=\beta$ we again have a periodic orbit; if not, we can follow $\gamma^{\prime}$ and mark in $[$ the successive points $\beta_{1}, \beta_{2}, \ldots$ where $\gamma^{\prime}$ crosses $\sum$ as $t$ increases and we do the same as $t$ decreases obtaining $\beta_{-1}, \beta_{2}, \ldots$. When the system is integrable and the orbits lie on tori it is possible to find a plane of section which intersects all orbits. The process that we have just described can be vlewed as a mapping $\Phi: \sum \rightarrow\left[\right.$ which takes a point $p$ of the plane of section into a point $p^{\prime}$ of the same plane. This mapping is called a Poincare map. Repeated application of the mapping $\Phi(p), \Phi(\Phi(p))=\Phi^{2}(p)$, etc. in the case of a periodic orbit will yield a finite $n$ such that $\Phi^{n}(p)=p$. Then if we let $A$ denote the set of points $\left\{p, \Phi(p), \ldots, \Phi^{n-1}(p)\right\}$ any iterate of a point a $\epsilon$ A also belongs to $A$. This property is expressed by saying that the set A is invariant under the map. There are also lines which are invariant under the map, i.e. if $\Gamma$ is such a line then $\Phi(p) \in \Gamma$ for all points $p \in \Gamma$. As we shall see the existence and properties of the invariant sets of the poincare map are of prime importance for the understanding of the behavior of Hamiltonian systems with two degrees of freedom.

As an illustration of how such mappings work we consider the motion generated by the unperturbed Hamiltonian $H_{O}$ as given by Eq. (13a) in the case $\beta \neq 0$. 
The solution is then

$$
\begin{aligned}
& J_{1}=J_{1}^{0}, w_{1}(t)=t+w_{1}^{0}, \bmod 2 \pi, \\
& J_{2}=J_{2}^{0}, w_{2}(t)=\left(\nu+B^{2} J_{2}^{0}\right) t+w_{2}^{0}, \bmod 2 \pi,
\end{aligned}
$$

where the superscript 0 refers to the initial values at $t=0$.

We fix the energy and eliminate $\mathrm{J}_{2}$ using

$$
\mathrm{J}_{1}+v \mathrm{~J}_{2}+\frac{1}{2} B^{2} \mathrm{~J}_{2}^{2}=\mathrm{E}
$$

which gives two determinations $J_{2}^{\prime}\left(E, J_{1}\right)$ and $J_{2}^{\prime \prime}\left(E, J_{1}\right)$. Concentrating upon the determination

$$
J_{2}^{\prime}\left(E, J_{1}\right)=\frac{1}{\beta^{2}}\left(-i+\left(\nu^{2}-2 \beta^{2}\left(J_{1}-E\right)\right)^{\frac{1}{2}}\right)
$$

for values of $\mathrm{J}_{1}$ and $E$ which make $\mathrm{J}_{2}^{\prime}$ real we take the plane $\mathrm{w}_{2}=0$ as the plane of section in the 3-dimensional phase-space of $J_{1}, w_{1}, w_{2}$. The projection of an orbit on the $w_{1}-w_{2}$ plane looks as in Figure (la), the slope of the orbit being equal to

$$
\omega_{2}\left(J_{1}^{0}\right)=\nu+B^{2} J_{2}^{\prime}\left(E, J_{1}^{0}\right)
$$

Each time the orbit crosses the plane $w_{2}=0$ we obtain a value for $w_{1}$ that we mark on the corresponding segment $\mathrm{J}_{1}^{*}=\mathrm{J}_{1}^{0}$ in the plane of section (Fig. $1 \mathrm{~b}$ ). Thus the study of a poincare map associated with an integrable system is fairly simple: the iterates $\Phi(\alpha), \Phi^{2}(\alpha), \ldots$ of a point $\alpha=$ $\left(w_{1}^{0}, J_{1}^{0}\right)$ are all located on the segment $0 \leq w_{1} \leq 2 \pi$ of the straight line $J_{1}=J_{1}^{0}$.

Note that if the problem is formulated in terms of the ordinary coordinates and momenta the invariant lines of a 2-degree of freedom integrable syslem are no longer straight. Indeed, suppose that the plane $q_{2}=q_{2}^{0}=$ constant is an appropriate surface of section. Then these lines are defined by the energy integral $\mathrm{H}(\mathrm{q}, \mathrm{p})=\mathrm{E}$ and the additional first integral $F_{1}(q, p)=c$ in which $q_{2}$ is set equal to $q_{2}^{0}, E$ is kept constant and $c$ is the 


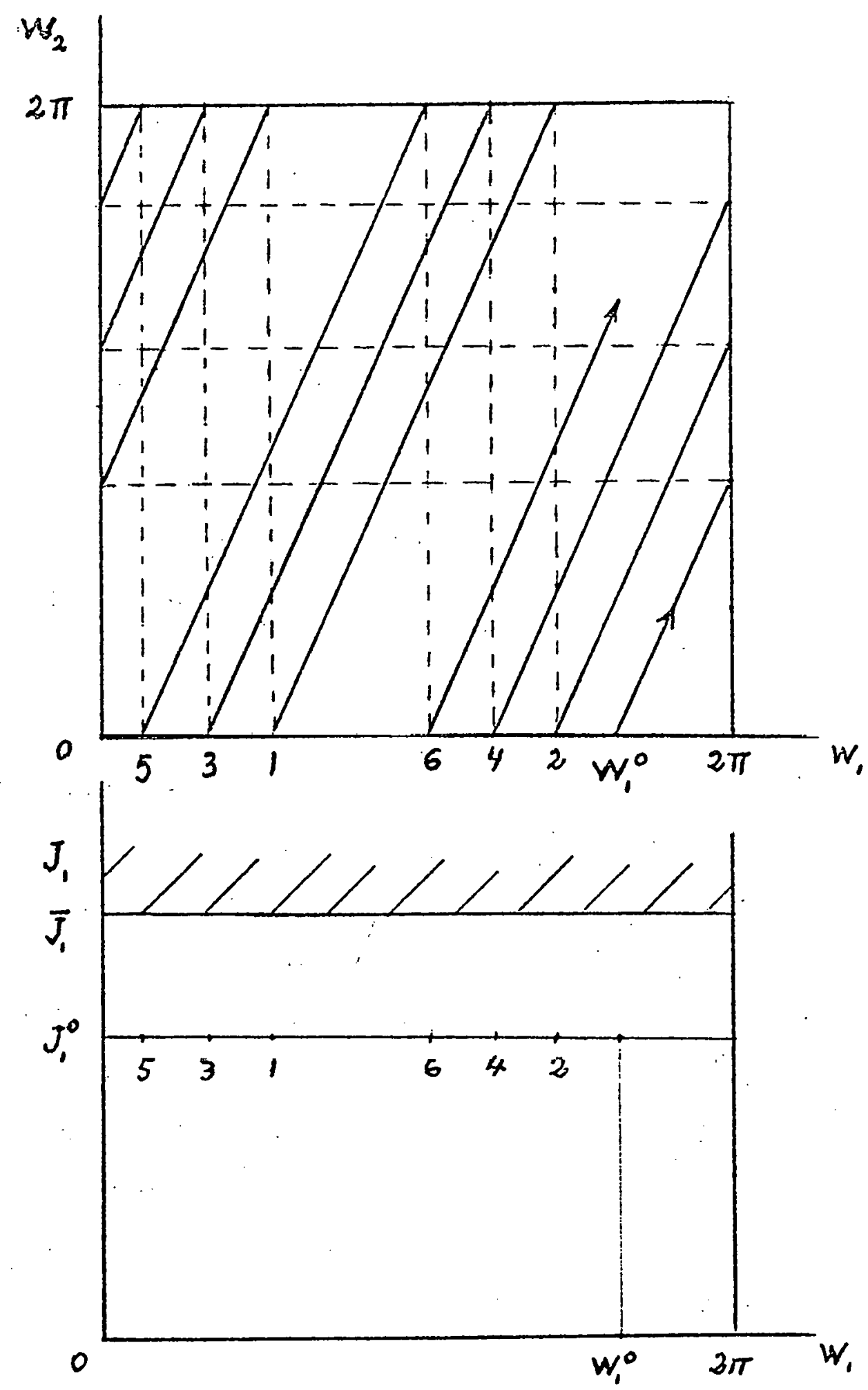

(a)

(b)

Fig. 1

$-1.5-$ 
parameter labeling these lines. These two equations in three unknowns $q_{1}$. $\mathrm{p}_{1}, \mathrm{p}_{2}$ are equivalent to a single equation in $\mathrm{q}_{1}, \mathrm{p}_{1}$, say, which depends on $C$ and therefore defines the one-parameter family of invariant lines. It is thus clear that in general these lines are not straight:

When a small perturbation is taken into account. things become extremely complicated if the perturbed system is not integrable. Returning to the action-angle variable formulation, by the KAM theorem there are still continuous smooth lines in the $w_{1}-J_{1}$ plane which are invariant under the motion just like the segments $J_{1}=J_{1}^{0}$ where invariant in the integrable case. These invariant lines fill most of the area available in the strip $0 \leq w_{1} \leq 2 \pi$ but not completely: there are gaps between them which cover a finite, although small, area.

Within these gaps one finds two kinds of motions: 1) first there are periodic motions which come from certain periodic motions of the unperturbed system, i.e. although the invariant tori corresponding to commensurable frequencies for $\varepsilon=0$ are destroyed some of the periodic orbits that they carry survive the perturbation; 2) the rest of the motions are not periodic and display a genuine random behavior.

A heuristic explanation for the appearance of these gaps can be given:

As already indicated, in order for the series $S\left(q, p^{\prime}\right)$ to converge it is necessary that none of the ratins $\omega_{i} / \omega_{f}, i \neq j$, of the eomponents of the frequency vector $w$ be a rational number. This requirement ic not serious, however, for in all probability it will be fulfilled for an wicked at random since the set of rational numbers has measure zero. Now, in expression (9) for the coefficient $s_{k}$ the numerator depends solely on the Fourier coeffiaicnt 
$h_{k}$ which, as is well known, tends to. zero exponentially with $|k| \equiv \sum_{l}^{n}\left|k_{i}\right|$ under the analytic assumptions made about $H_{1}$. There is then the question whether there exist frequencies os such that the small denominators $(k, w)$ tend to zero slowly enough so that the series for $S$ converges. It was Kolmogorov's great contribution to point out that a classical theorem of the theory of the so-called Diophantine approximations (26) provides a positive answer to this question. This theorem states that given a frequency $\omega$ it is possible to find a number $\mathrm{K}$ depending on $w$ but not on $\mathrm{k}$ such that the inequality

$$
|(k, w)| \geq K(w)|k|^{-(n+1)}
$$

holds for all $k \neq 0$. Using this fact it is then easy to show that given an uncommensurable w there is a number $\mathrm{K}$ for which the series for $\mathrm{S}$ converges. However, the convergence of the series for $S$ is not enough since in the perturbation scheme $s$ is but the first of an infinite sequence of characteristic functions $s=s^{(1)}, s^{(2)}, \ldots$ producing a sequence of angle-independent Hamiltonians ${ }_{0}^{(1)},{ }_{0}^{(2)}, \ldots$, whose calculation introduces an additional difficulty: at each level $s$ of approximation the frequency is different since it is given by $\omega=\partial \mathrm{H}_{0}^{(\mathrm{s})} / \partial \mathrm{p}(\mathrm{s})$.

Arnold found that in order for all these series to converge w must belong to a certain set $\Omega$ of positive measure.

In the space of all admissible frequencies the complement $\Omega_{c}$ of $\Omega$ has a finite measure for $\varepsilon>0$ which tends to zero with $\varepsilon$. What differentiates the frequency vectors in $\Omega$ from those in $\Omega_{c}$ is that the irrational ratios of their components, i.e. $w_{i} / w_{j}$ cannot be approximated by rational numbers as well as such ratios associated with the frequency vectors belonging to $\Omega_{C}$. What is meant here is that given $\mu>0$ the inequality $|(k, \omega)| \leq \underline{\mu}$ can be satisfied 
when $w$ is in $\Omega_{c}$ for values of $|k|$ much smaller than when $w \epsilon \Omega$. For instance, consider the two irrational numbers: $N \equiv(\sqrt{5}-1) / 2=0.618033988749 \ldots$ (the golden mean), and $\pi / 5=0.6283185308 \ldots$. If in $(k, \omega)$ we take $k_{1}=71, k_{2}=-113$ we get $71-113 \times(\pi / 5)<7 \times 10^{-6}$ for $|k|=|71|+|-113|=184$. On the other hand, in order to have $\left|k_{1}+k_{2} N\right|<7 \times 10^{-6}$ it can be shown using the theory of continued fractions that it is necessary to go up to $|k|=121393$, corresponding to $k_{1}=46368, k_{2}=-75025$. Now, to each frequency in $\Omega$ there correspond a finite number of tori $p^{(\infty)}$ obtained by inverting the equation $\omega=\partial \mathrm{H}_{0}^{(\infty)} / \partial \mathrm{p}{ }^{(\infty)}$ and which are in nne-toune currespondence with tori $p(\omega)$ of the unperturbed system through the inverse of the canonical transformation $q, p \rightarrow q^{(\infty)}, p^{(\infty)}$. These are the tori which are preserved under the perturbation and they fill most of phase-space since the volume they occupy is proportional to the measure of $\Omega$. On the contraxy, the other tori, i.e. those corresponding in the same fashion to the frequencies in the complement $\Omega_{c}$ disappear under the perturbation leaving vacant a volume vanishingly small with $\varepsilon$ like the measure of $\Omega_{c}$.

Finally, because any neighborhood of a frequency in $\Omega$ contains frequencies in the complement $\Omega_{r}$, there are disappearing tori in the neiqhborhood of any of the survivors, i.e. the vntume oscupied by the disappearing tori is the union of thin gaps between surviving tori which are present everywhere in phase-space. As mentionned before, these gaps are filled with orbits of a hihgly complicated nature, in contrast with the orbits lying on the preserved tori which give rise to quasi-periodic motions.

nt the present time, lile must powerful tool available for the study of these complicated orbits is Poincare's method of section. For more than two degrees of freedom his method is difficult to apply the "surfaces" of 
section having $2 n-2$ dimensions; but for $n=2$ they are usual two-dimensional surfaces so that their corresponding Poincare map is not as difficult to study. Many fascinating generic properties of two-degree of freedom Hamiltonian systems can then be derived from those of their associated poincare map as we shall see.

\subsection{Poincare Maps and Area-Preserving Mappings}

If $\mathrm{x}$ and $\mathrm{y}$ are appropriate curvilinear coordinates defined on a surface of section $\Sigma$ of a two-degree of freedom, analytic Hamiltonian system, the Poincare map $\Phi$ takes a point $(x, y)$ into a point $\left(x^{\prime}, y^{\prime}\right)$, i.e.

$$
\Phi: x^{\prime}=f(x, y), y^{\prime}=g(x, y),
$$

where $f$ and $g$ are analytic functions. For non-integrable systems these functions cannot be determined; yet, it turns out that they possess a generic property from which a surprisingly large number of qualitative features of the system can be found. This property is that there always exists a certain function $Q(x, y)$ such that the integral $\int Q(x, y) d x d y$ has the same value when evaluated over any region of $\sum$ as over its image under $\Phi(27)$. Clearly this property can be expressed equivalently by the equation

$$
Q(x, y)=Q\left(x^{\prime}, y^{\prime}\right) \frac{\partial\left(x^{\prime} y^{\prime}\right)}{\partial(x, y)}=Q\left(x^{\prime}, y^{\prime}\right)\left(\frac{\partial f}{\partial x} \frac{\partial g}{\partial y}-\frac{\partial g}{\partial x} \frac{\partial f}{\partial y}\right)
$$

that the functions $f$ and $g$ satisfy. It turns out that the very existence of a surface of section implies that the function $Q$ does not vanish in the region of definition of the mapping $\Phi$. As a consequence, there exists an invertible change of variables $(x, y) \rightarrow(x, y)$ such that in terms of the new variables the above integral reduces to $\int \mathrm{dXdY}, i . e$. in the plane of $X$ and $Y$ the poincare map preserves areas in the usual sense $(s-8)$. This is why it is usually stated that the Poincare map is area preserving although, in reality, this is rarely 
the case since in general the function $Q$ is not constant on an arbitraxy surface of section. This abuse of language is not serious however since the generic properties of an area-preserving mapping of the plane $X, Y$ are essentially topological in nature, i.e. they are preserved under the inverse transformation $(x, y) \rightarrow(x, y)$. It is therefore sufficient - and much easier - to investigate these properties in the case of an area-preserving transformation of the plane, and this is what we are going to do in the following (s-9).

To begin with, we look at the behavior of the map $\Phi$ in a neighborhood of one of its fixed points which we assume to be at the origin $x=y=0$. If. $z$ denotes the vector of components $x, y$ we can write Eqs (15) as

$$
\Phi: z^{\prime}=A z+h(z)
$$

where $A$ is a constant matrix and $h$ a series in $x$ and $y$ lacking constant and linear terms. The area-preserving property of $\Phi$ implies that $\operatorname{det} A=1$. Fixed points are classified according to the nature of the eigon values of the matrix A. For the linearized mapping

$$
\Phi_{L}: z^{\prime}=A z
$$

an arbitrary neighborhood of the origin is filled with invariant curves whose configuration depends upon the nature of the eigenvalues $\lambda_{1}, \lambda_{2}$ of $A$, i.e. un 1ts trace alone since the characteristic equation is of the form

$$
\lambda^{2}-(\operatorname{trace} A) \lambda+1=0 .
$$

a) trace $A>2$ : (real positive roots $\alpha, \alpha^{-1}$ ) the invariant curves are hyperbolae. The iterates $1,2, \ldots$ of a point all lie on the branch of the particular hyperbola going through it. The configuration is as shown in Fig. (2a) where, as in the following figures, the numbers indicate the locations of the successive iterates under repeated application of $\Phi_{L} \cdot$ The fixed point is then called hyperbolic (without reflertion). 


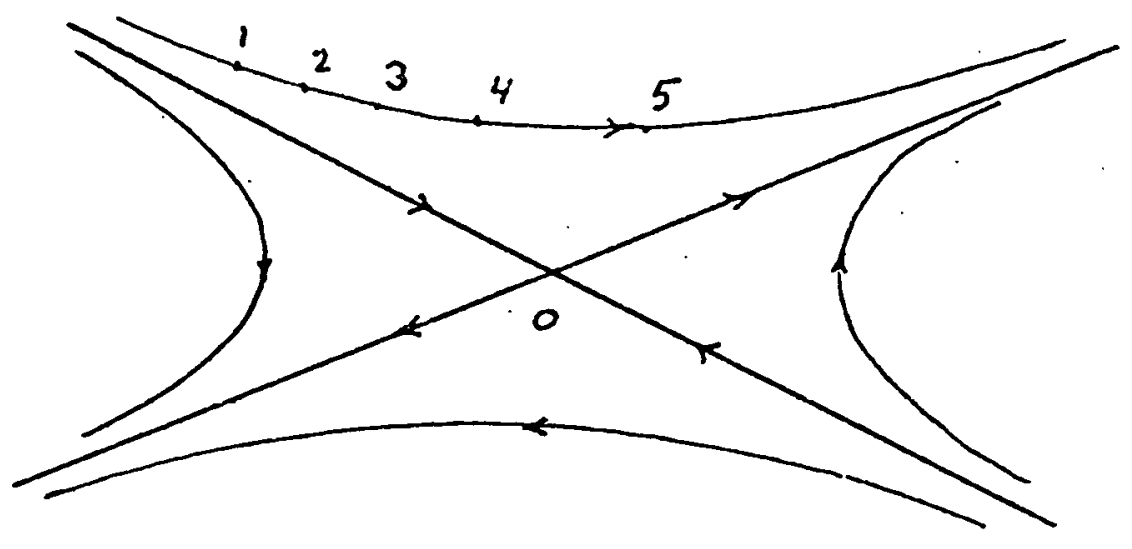

(a)

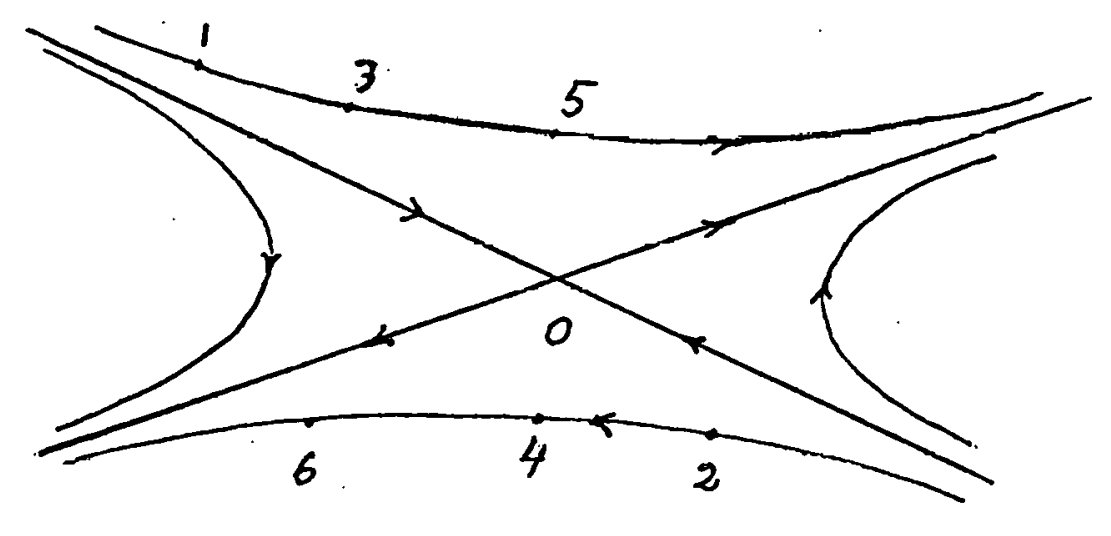

(b)

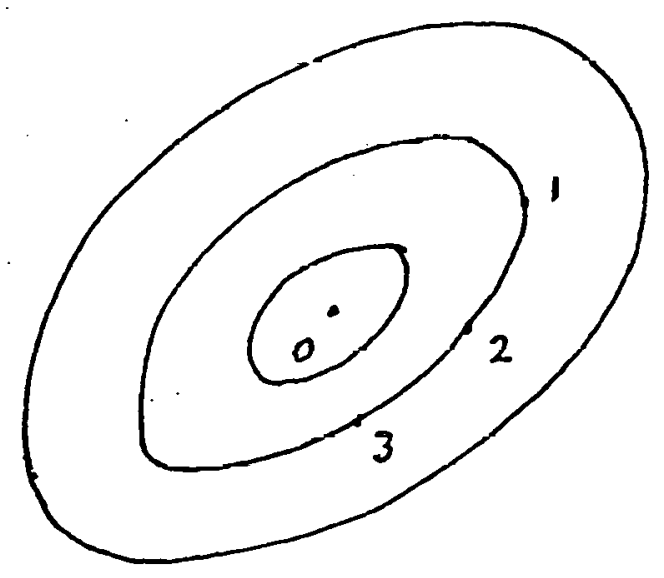

Fig. 2

(c) 
b) trace $A<-2$ (real negative roots $\left.-\beta,-\beta^{-i}\right)$ : the configuration is the same but the successive points jump alternatively from one branch of the hyperbola to the other as shown in Figure (2b). The point is called hyperbolic. with reflection.

c) $\quad-2<\operatorname{trace} A<2$ (complex conjugate roots $e^{ \pm i} \gamma, \gamma$ real): the invariant curves are concentric ellipses as shown in Figure (2c). The fixed point is called elliptic.

d) trace $A= \pm 2$ : this is an exceptional situation in which the point is called parabolic without reflection if $\lambda_{1}=\lambda_{2}=1$, and with reflection if $\lambda_{1}=$ $\lambda_{2}=-1$

Birkhoff (14) and Moser (44) have shown that the configuration of the invariant curves in a sufficiently small neighborhood of a hyperbolic point is the same for a non-linear mapping as that of its linear part. There is an analytic function $G(x, y)$ which is constant along the invariant curves. This implies that the corresponding Hamiltonian system possesses a first integral in a small region of phase-space surrounding the periodic orbit corresponding to the hyperbolic fixed point. Note that this first integral is not "isolating" in the sense that the corresponding level surfaces do not cast out regions of phase-space where orbits would remain forever. The behavior of the motions away from the periodic orbit is remarkably complicated as we are going to show now.

Among the invariant. surves defined hy $G(x, y)=$ sonstant two go through the fixed point where they are tangent to the asymptotes of the hyperbolae of the linearized map. One of them denoted $w^{\mathbf{s}}$ is stable in the sense that $\Phi^{k}(p) \rightarrow 0$ as $k \rightarrow \infty$ for all $p \in W^{S}$, while the other $w^{u}$ is unstable, i.e. $\lim \Phi^{k}(p) \rightarrow 0$ as $k \rightarrow-\infty$. Suppose $w^{s}$ and $w^{u}$ intersect transversally 
at a point $P$. Then it can be shown that they intersect at an infinity of points which are the iterates $P_{i}, i= \pm 1, \pm 2, \ldots$ of the mapping.' Now consider the sequence $P, P_{1}, P_{2}, \ldots$; it is infinite, lies on the arc $P O$ of $w^{s}$, and has $O$ as limit point. Hence the distance between two consecutive points tends to zero as $i$ tends to infinity. Since the area between the $\operatorname{arc} P_{i} P_{i+1}$ of $w^{S}$ and the corresponding arc of $\mathrm{w}^{\mathrm{u}}$ is constant under the mapping it therefore follows that the dimension of the surface enclosed within these arcs in the direction normal to $\mathrm{w}^{\mathbf{S}}$ must increase indefinitely producing the configuration sketched in Figure (3a) since $\mathrm{w}^{\mathrm{u}}$ cannot intersect itself. Furthermore $\mathrm{w}^{\mathrm{s}}$ behaves in the same way and therefore intersects $w^{4}$ in an infinity of points as shown in Figure (3b). All the points where $\mathrm{w}^{\mathrm{S}}$ and $\mathrm{w}^{\mathrm{u}}$ intersect are called homoclinic and it is seen that the existence of one implies the existence of an infinity. Note that the orbit going through $P$ and its iterates approaches the unstable periodic orbit going through $O$ as the time tends to $\infty$ and $-\infty$. It is called a homoclinic orbit.

Because of the intricate configuration of the invariant curves $\mathrm{w}^{5}$ and $w^{\mathrm{u}}$ the behavior of the orbits close to the homoclinic orbits seems to defy analysis. Hadamard (45) and Birkhoff (14, Vol. 2, p. 653) realized however that these motions can be put into one-to-one correspondence with sequences of symbols. This was elaborated upon by Morse and Hedlund (46) who laid down the foundations of what is called symbolic dynamics. Morc rcocntly this method of analysis has made considerable progress thanks to the works of Smale (47), Alekseef (48, 49) and others (cf 18).

Alekseef has very clearly explained that (48) "the basis of symbolic dynamical methods is the association of infinite words in a certain alphabet 


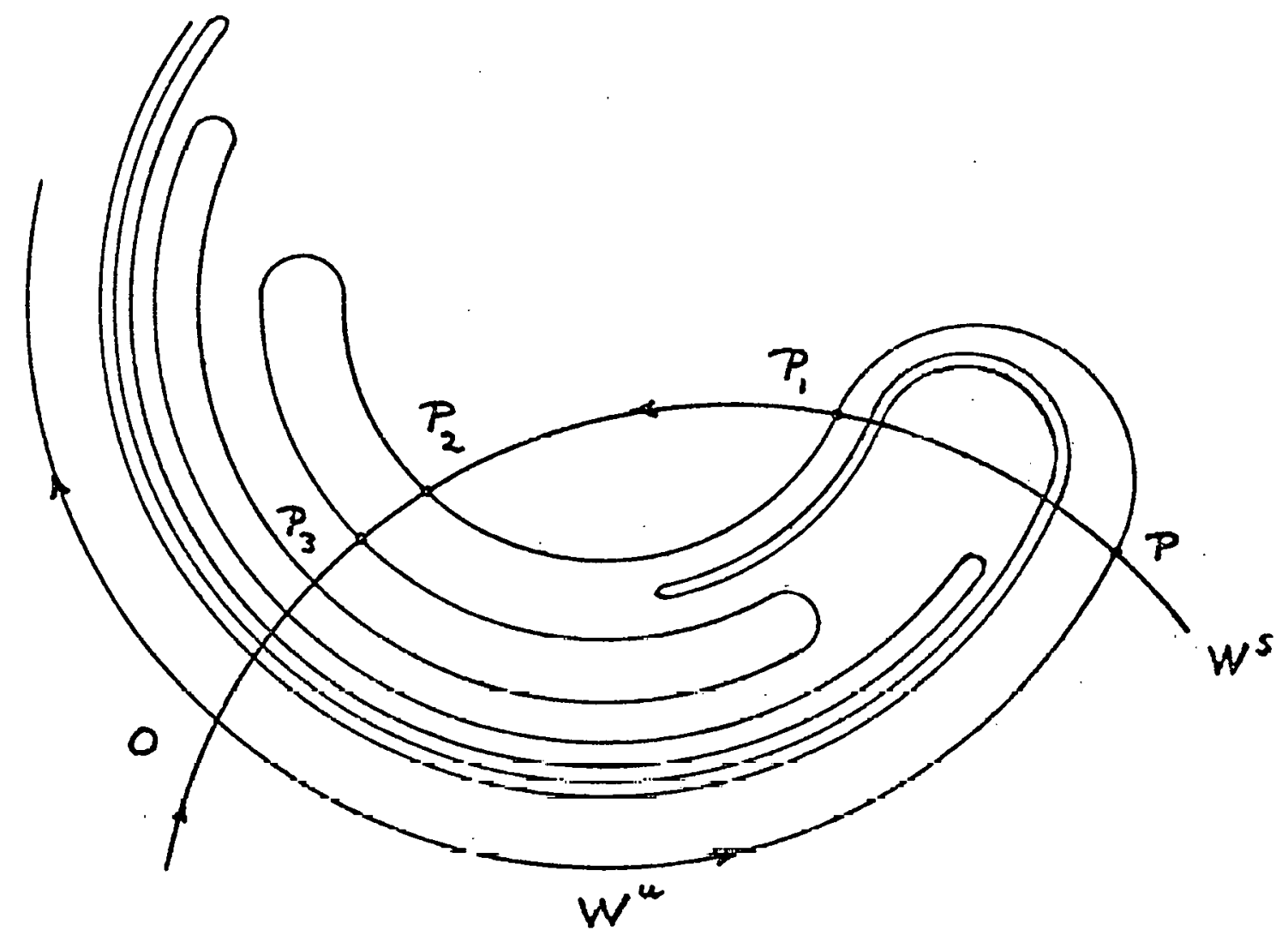

Fig. $3 a$. 


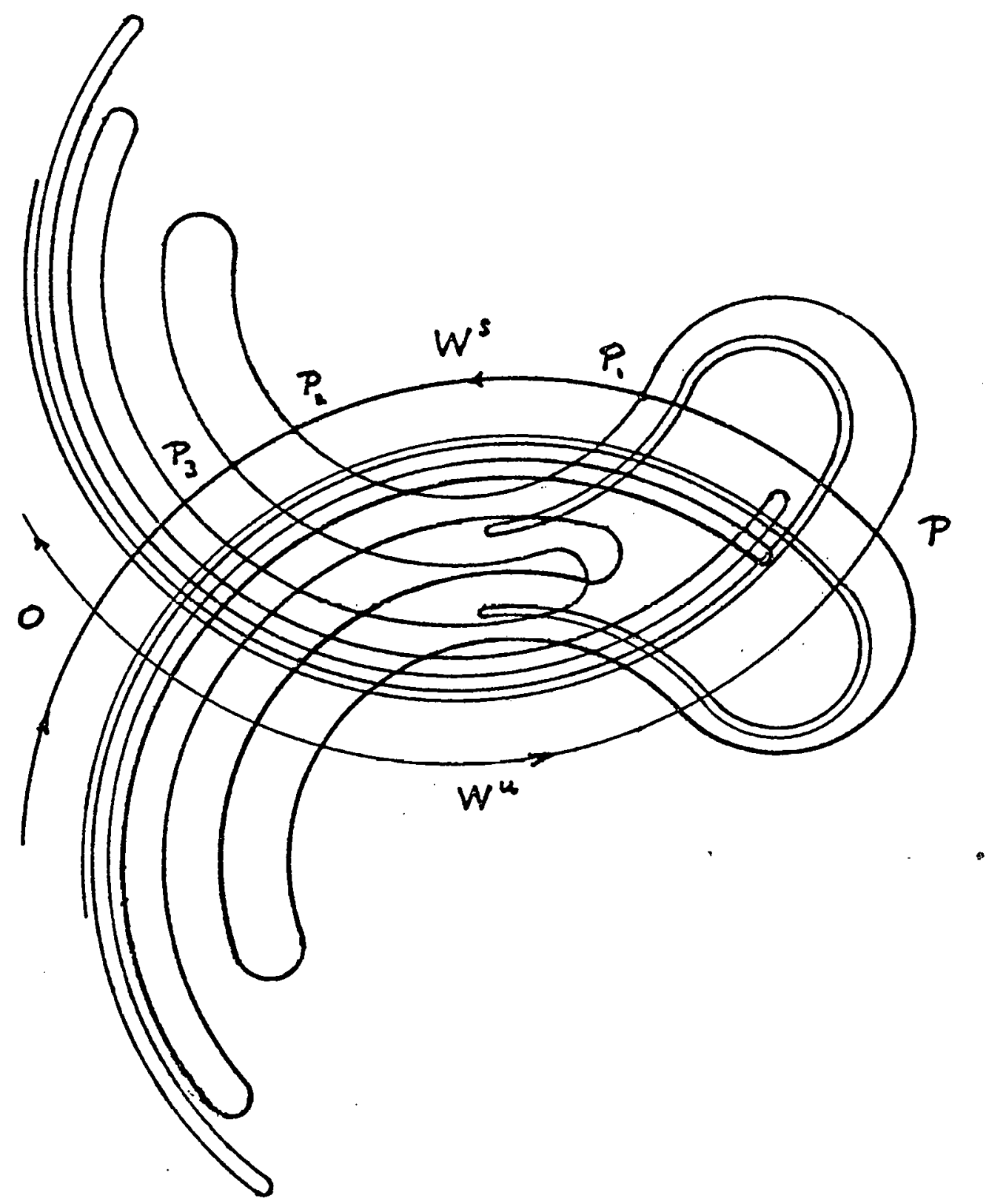

Fig. $3 b$ 
with the trajectories of a dynamical system. This is done by choosing a certain partition of a surface of section, the elements of the partition being denoted by letters. By observing those elements in which a trajectory falls as it returns successively to the surface of section, we obtain the word corresponding to the trajectory. It is clear that the fundamental mapping of the surface of section*, the shift transformation, corresponds to the shift by one letter to the left. This scheme has the following physical interpretation. To letters, i.e. elements of the partition, correspond outcomes of a certain experiment, the "macro-measurements"; to a word corresponds a sequence of results of repeated measurements in a system evolving with time"..

The analysis of the properties of the poincare map in the vicinity of a homoclinic point is very difficult even with the help of symbolic dynamics. It is however possible to understand the essentials of the mathematical arguments involved using the close analogies which exist between the Poincare map and certain known mappings whose properties can be derived in a heuristic way without much difficulty. The best known mapping in this category is probably smale's horseshoe map that we proceed to describe and analyze (50). smale's horseshoe map is a mapping of a square $Q$ into the plane which can be detined informally as follows (see Figure 4): imagine that $Q$ is made of fresh dough and mark a point $p$ on it. Then stretch $Q$ horizontally while letting its height shrink (holes and tears are not allowed!). With enough stretching we can obtain a strip of dough $1 \mathrm{nng}$ onough to be bent into the shape of a horseshoe that we position over the square as shown in tho figuro. By detinition, the new location of $p$ after these manipulations is the image $\Phi(p)$ of $p$ under the mapping.

*I.e. One forward application of the Poincare map. 

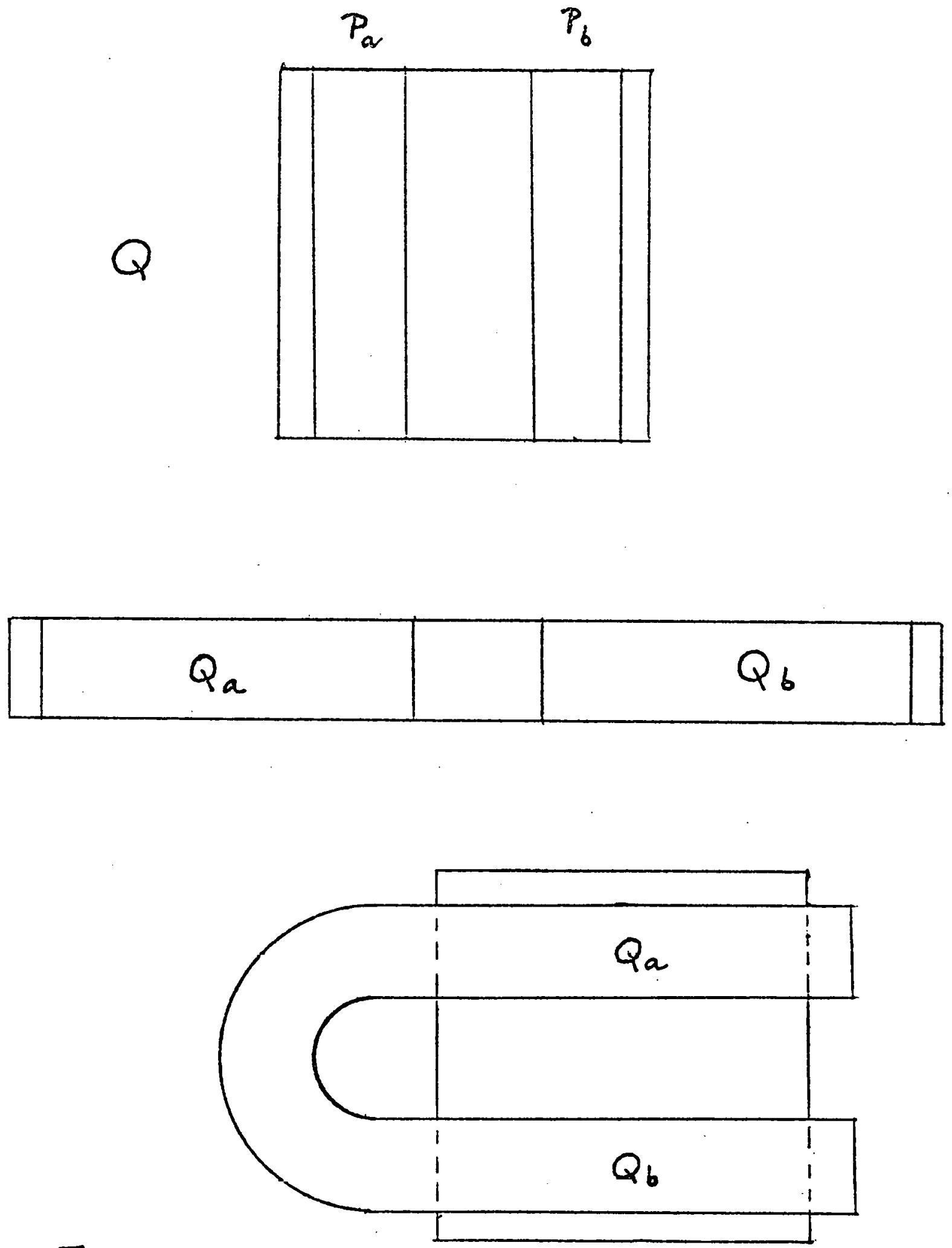

Fig. 4 
Note that $\Phi(p)$ may fall within $Q$ or not and that, in the latter case, its image under $\Phi$ is not defined. However, there exists a subset $\Lambda$ of $Q$ with the property that all the: iterates $\Phi^{n}(p)$ of any $p \in \Lambda$ belong to $Q$ for $\mathrm{n}=0, \pm 1, \pm 2, \ldots$. The existence of $\Lambda$ can be ascertained as follows. First, consider the forward iterates of the square. In order for $p \in Q$ to belong to $\Lambda$ it is necessary that its image $\Phi(p)$ fall in either of the rectangles $Q_{a}$ and $Q_{b}$ for, otherwise, its second iterate would not be defined. Hence $\Phi(p) \in Q$ only if $p$ belongs to either of the preimages $P_{a}=\Phi^{-1}\left(Q_{a}\right)$ and $P_{b}=\Phi^{\prime \prime}\left(Q_{b}\right)$ of $Q_{a}$ and $Q_{b}$, respectively. It is easy to see by reversing the above construction and thus bringing the horseshoe shaped piece of dough back into the shape of $Q$ that $P_{a}$ and $P_{b}$ are vertical strips as shown in the figure.

Now assume that $p \in P_{a}$ or $P_{b}$ so that $\Phi(p) \in Q_{a}$ or $Q_{b}$ and apply $\Phi$ a second time. As we have just seen, for $\Phi^{2}(p)$ to fall within $Q \Phi(p)$ must also be in $P_{a}$ or $P_{b}$. Hence $p$ must lie in the preimage of one of the four little rectangles $Q_{i} \cap P_{j}, i, j=a$ or $b$, and $i t$ is easy to verify that the preimage of each of these is a vertical strip $P_{i j}$ which is a subset of $P_{i}$, i.e. $P_{i j}=\Phi^{-1}\left(Q_{i} \cap P_{j}\right) \subset P_{i}($ see Figure 5$)$.

Observe that the image of $\ell_{i^{\prime}} i:=a$ or $b$, is a horseshoe lying in $\Phi(Q)$ whose intersection with $Q$ consists of two horizontal strips $Q_{i j}, j=a$ or $b$. Clearly we can label these four strips in such a way that $Q_{i j} \subset \mathcal{Q}_{i} \subset Q$ (see Figure 6). If we apply $\Phi$ once more $\Phi^{3}(p)$ will fall in $Q$ provided $\Phi^{2}(p)$ belorigs to $\mathrm{P}_{\mathrm{a}}$ or $\mathrm{P}_{\mathrm{b}}$. As before, it is readily seen. that for this to happen $\mathrm{p}$ must belong to one of the eight vertical strips

$$
P_{i j k}=\Phi^{-2}\left(Q_{i j} \cap P_{k}\right) \subset P_{i j} \subset P_{i}, i, j, k=a \text { or } b .
$$



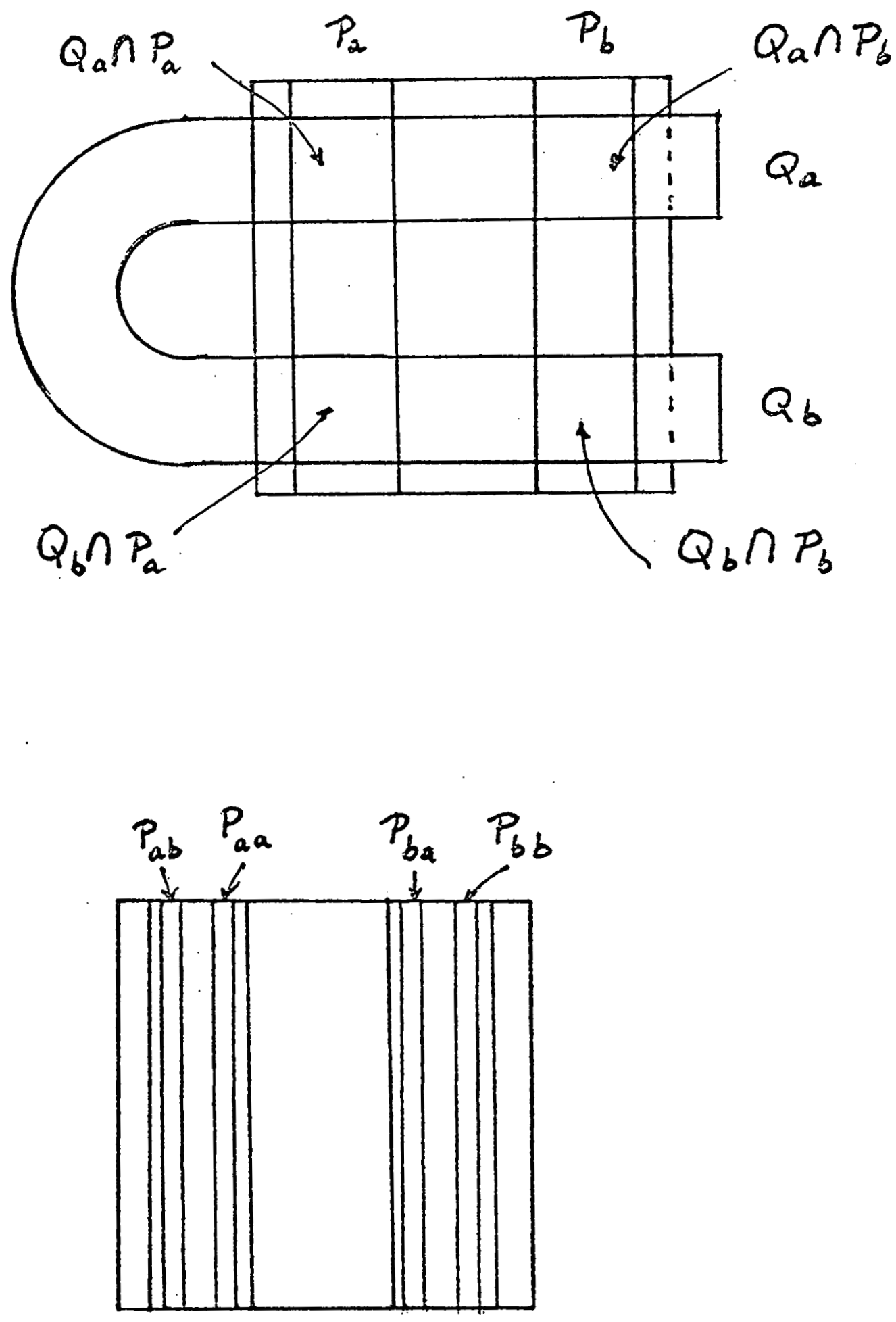

Fig. 5 

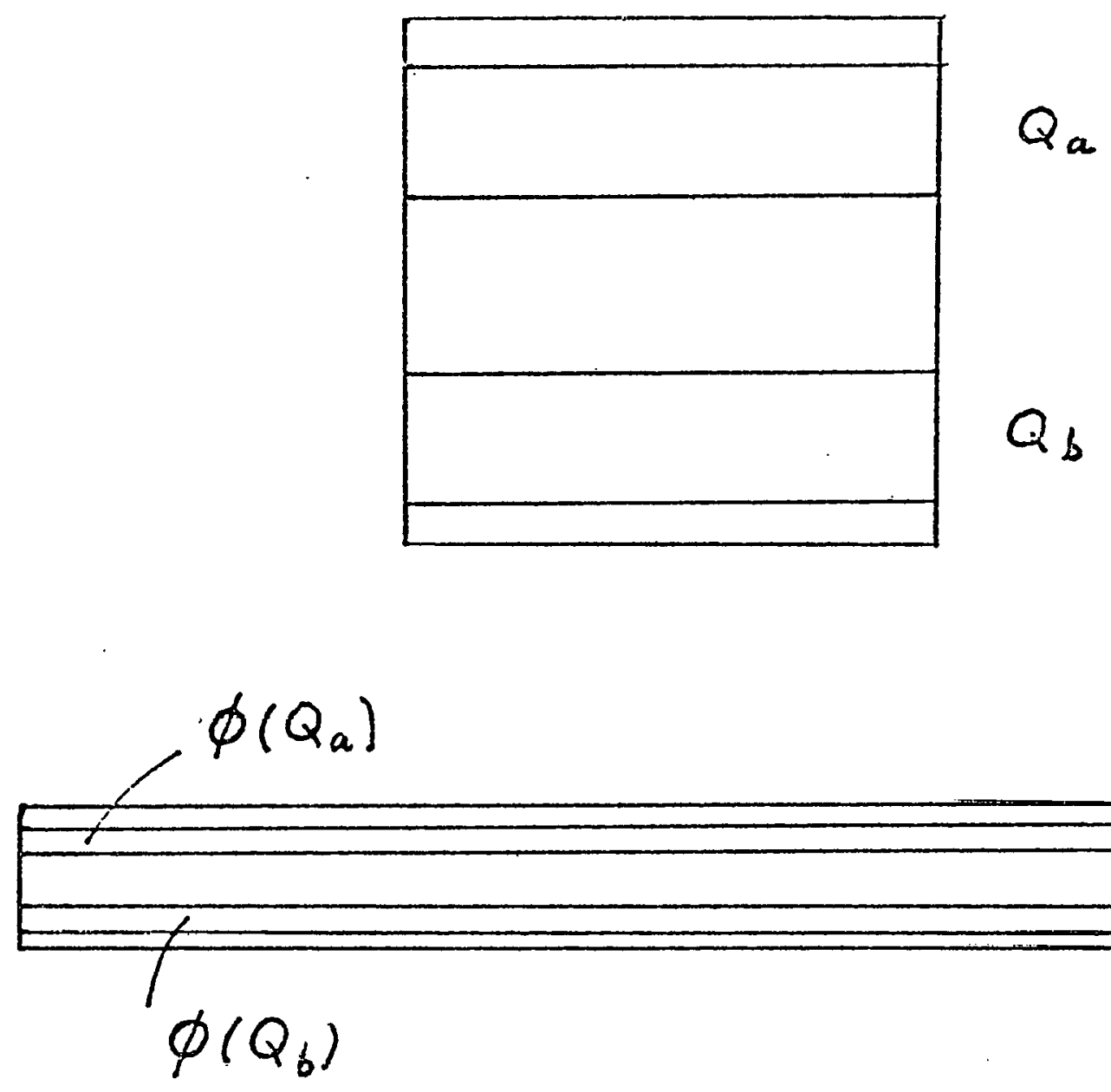

Fig. 6

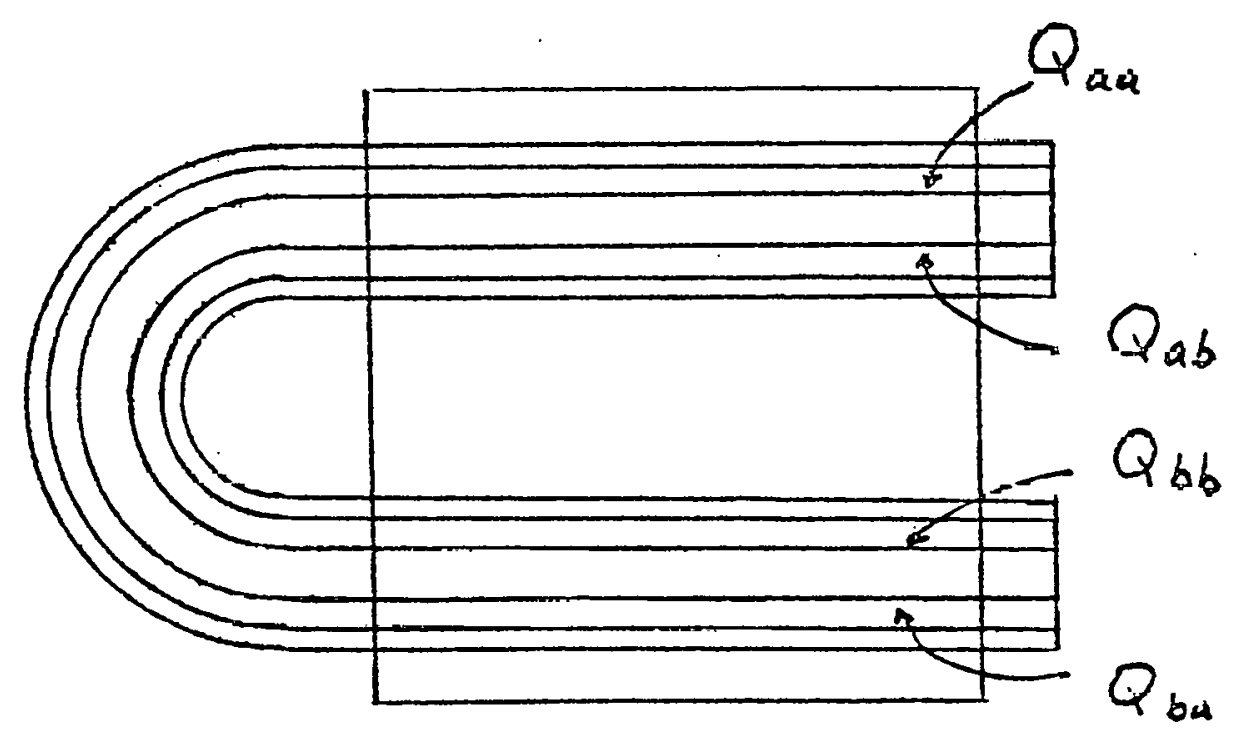


Finally, if we apply $\Phi$ an infinite number of times we obtain an infinite set $\Lambda_{1}$ of vertical strips of zero width since from the construction of $\Phi$ horizontal distances shrink by a factor smaller than one under the application of $\Phi^{-1}$. In other words we know at this stage that $\Lambda$ is a subset of an infinite set $\Lambda_{1}$ of vertical segments $P_{i j k} \ldots$ where $i, j, k, \ldots=a$ or $b$. From the way we have labeled the elements $P_{i j k} .$. of the set $\Lambda_{1}$ we see that they are in one-to-one correspondence with the elements of the set of infinite sequences of two symbols $\{i, j, k, \ldots\}$, where $i, j, k, \ldots=$ $a$ or $b$. Because the elements of the so-called ternary set of Cantor are also in one-to-one correspondence with this set of sequences, the set $\Lambda_{1}$ is equivalent to this Cantor set and, for this reason, is itself called a Cantor set (see Appendix D).

Next we consider the backward iterates $\Phi^{-n}(p), n=1,2, \ldots$ of a point $p \in Q$ under the inverse mapping (see Figure 6).

From the foregoing $\Phi^{-1}(p)$ is defined only if $p$ belongs to $Q_{a}$ or $l_{b}$ ' $\Phi^{-2}(p)$ is defined only if $p$ belongs to either of the four horizontal strips $l_{i j} j^{\prime}, j=a$ or $b$, and, in the limit, $\Phi^{-\infty}(p)$ is defined only if $p$ belongs to one of infinitely many horizontal segments $Q_{i j k} .$. whose set we denote by $\Lambda_{2}$. Just like $\Lambda_{1}, \Lambda_{2}$ is a Cantor set.

It is now obvious that the set $\Lambda$ that we are looking for is the intersection of $\Lambda_{1}$ and $\Lambda_{2}$, that is the set of points of intersection of the vertical segments of $\Lambda_{1}$ with the horizontal segments of $\Lambda_{2} \cdot \Lambda$ is thus the product of two Cantor sets, i.e. a Cantor set in two dimensions, whose points are in one-to-one correspondence with the elements of the set $s$ of doubly infinite sequences of two symbols. Indeed a point $p \in \Lambda$ is at the intersection 


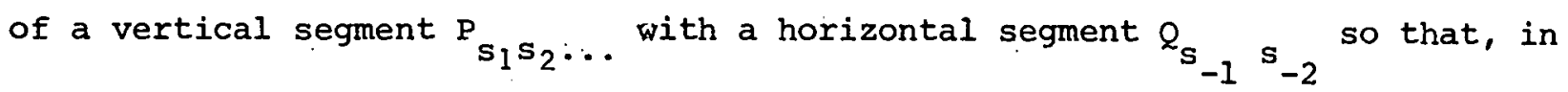
obvious notation, the infinite: sequence $s=\left(\ldots s_{-2} s_{-1} \cdot s_{1} s_{2} \ldots\right)$ is uniquely associated with $\mathrm{p}$ and vice-versa.

Each point of $\Lambda$ can therefore be labeled by its corresponding sequence $s$ and it is clear that the sequence corresponding to $\Phi(p)$ is obtained from $s$ by simply shifting the "decimal" point by one symbol to the left.

Shifts over doubly infinite sequences of symbols are commonly used in probability theory since they constitute convenient representations of chance processes like the consecutive outcomes of spins of a roulette wheel. They are usually callē Bernoulli shifts.

The set $\Lambda$ has the following important properties:

1) it contains a non-denumerable set of periodic points of $\Phi$ with arbitrarily large period. These periodic points evidently corresponds to those doubly infinite sequences of $\mathrm{S}$ which are periodic.

2) an arbitrarily small neighborhood of a point of $\Lambda$ contains periodic points of $\Phi$. In topological language this property is expressed by saying that the set of periodic points of $\Phi$ is dense in $\Lambda$.

3) the set $\Lambda$ is hyperbolic by which is meant the following: if we consider the effect of $\Phi$ on a small neighborhood of a point p€ $\Lambda, \Phi$ contracts distances in the vertical direction and expands them in the horizontal direction. This is analogous to the behavior of a Poincare map at a hyperbolic point, hence the terminology. Such a behavior is often referred to as a hyperbolic splitting. The conditions under which it occurs can be expressed in a precise manner for higher dimensional mappings in terms of the spectrum and eigenspace of the Jacobian matrix of the mapping: if we denote by $D \Phi$ the linear map generated by this matrix these conditions are that the eigenspace can be split into independent components $E^{\mathbf{S}}$ and $E^{\mathbf{u}}$ such that $D \Phi$ contracts on $E^{\mathbf{S}}$ and expands 
on $\mathrm{E}^{\mathrm{u}}$. The importance of this notion of hyperbolicity stems from the fact that mappings which possess an invariant hyperbolic set often generate random behavior under succéssive iteration and are ergodic.

We are now ready to discuss the crucial physical consequences of these properties: if we view the horseshoe map as the Poincare map of a discrete dynamical system, the behavior of the system is random at any macroscopic level of description. The dynamical system that we have in mind is defined as follows: the possible states of the system are represented by the points of the plane with the law of evolution

$$
p_{n+1}=\Phi\left(p_{n}\right) \text {, }
$$

i.e. if the system is in the state $p_{n}$ at time $n, n$ an integer, its state at time $n+1$ is the image of $p_{n}$ under $\Phi$.

suppose now that we observe this system for a limited time and with limited precision. We can for instance fix our attention on the two vertical strips $P_{a}$ and $P_{b}$ and record in which of these $p_{n}$ falls at times $n, n+1, \ldots$ $\mathrm{n}+\mathrm{k}$, thereby obtaining a string of $\mathrm{k}$ letters $\mathrm{s}_{1} \mathrm{~s}_{2} \cdots \mathrm{s}_{\mathrm{k}}\left(\mathrm{s}_{\mathbf{i}}=\mathrm{a}\right.$ or $\left.\mathrm{b}\right)$. From the foregoing discussion the actual orbit either belongs to $\Lambda$ or not but we have no way of telling which is the case from our limited observations. Worse, even if we knew that the actual orbit is in $\Lambda$ we could not tell anything about its future history because there is a continuum of possibilities corresponding to the infinite subset of sequences which contain a string of $k$ letters identical to the string that we have recorded. In other words, no matter how large $k$ is, we cannot predict in which of $P_{a}$ or $P_{b} P_{n+k+1}$ is going to fall the probability of either events being $1 / 2$ (provided of course that $p_{n} \in \Lambda$ ).

We now return to the problem of the behavior of the poincare map in the neighborhood of a homoclinic point.

We shall content ourselves to state the results obtained by Smale, Moser, and Conley (cf 18, p. 99). 
Smale has shown that in any neighborhood of a homoclinic point there exists: an invariant set $I_{2}$ for some fixed power, say $\Phi^{l}$, of $\Phi$.such that, under the mapping $\Phi^{l}$, the points of $\mathrm{I}_{2}$ are in one-to-one correspondence with the doubly infinite sequences of two symbols. More generally, Conley has shown that the restriction to a fixed power of $\Phi$ can be relaxed in which case there exists an invariant set $I_{\infty}$ whose points are in one-to-one correspondence with the doubly infinite sequences of an infinity of symbols. The sets $I_{2}$ and $I_{\infty}$ have the same properties as the set $\Lambda$ of the horseshoe map. Consequently, not only is there an infinity of periodic points (a result already obtained by Birkhoff, of ref. 14, Vol. 2, p. 530) but, more importantly, the behavior of the Poincare map is of the same unpredictable nature as that of the horseshoe map. Now that we have given an idea of the complexity of the nelghborhood of a hyperbolic fixed point when homoclinic points are present we can indicate what happens in the neighborhood of an elliptic point.

The KAM theorem says that under a small perturbation most of the invariant ellipses surrounding an elliptic fixed point of the unperturbed system will be slightly deformed into closed invariant curves corresponding to the uncommensurable frequencies in the set $\delta^{(*)}$. In the gaps left uul by these invariant curves there necessarily are homoclinic points as shown by zchnder (51). These homoclinic points belong to hyperbolic points which occur in pairs with elliptic points. Each of these elliptic points is in turn surrounded by a similar configuration of invariant curves separated by gaps containing homoclinic, hyperbolic, and elliptic points, etc. in an endless fashion. To give an idea of the complexity of such a configuration we reproducc in Figure (7) the picture given by Arnold (12). Note that this picture corresponds to a

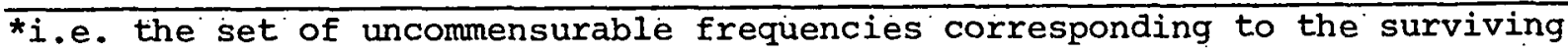
tori (cf p. 17) 


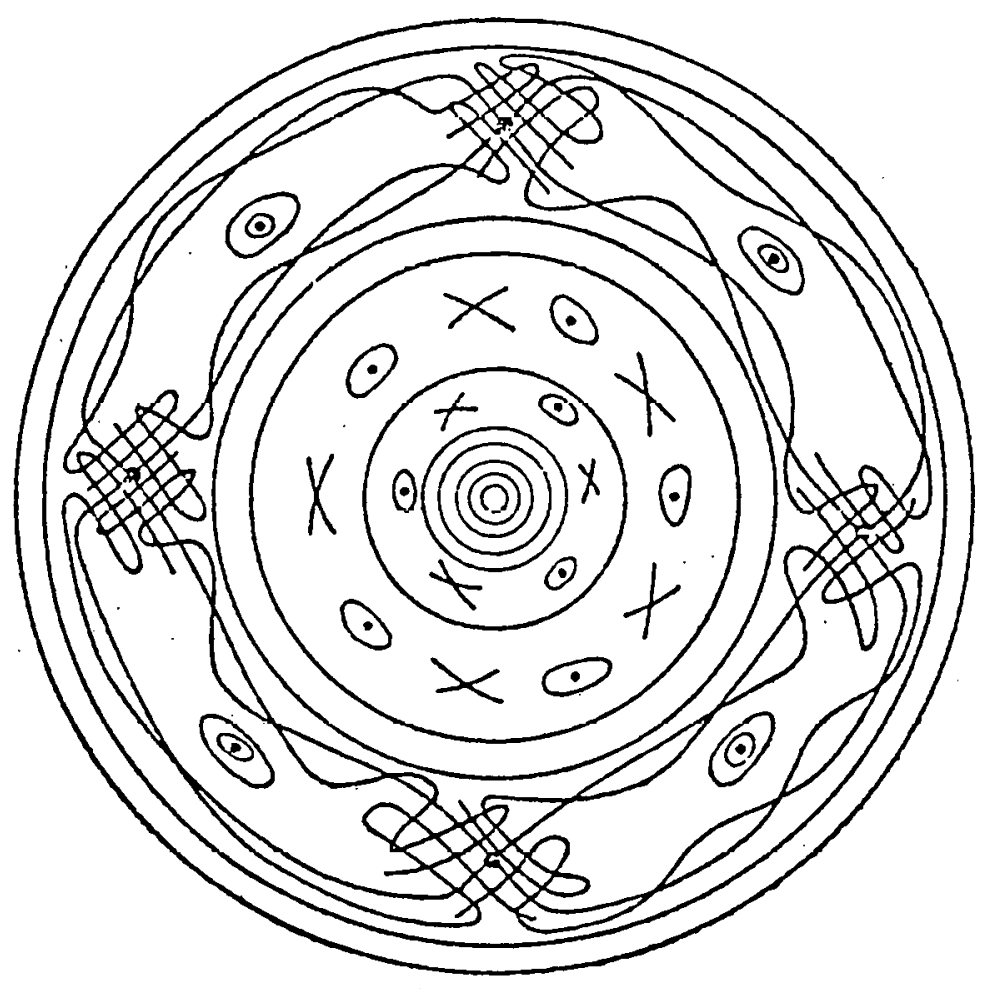

Fig. 7 
perturbation which is large enough for the gaps to be visible but so small that on the scale of the figure a definite geometric structure is still discernable. As $\varepsilon$ is increased however this structure eventually disappears completely in wide regions. The sequence of morphological changes which take place can be described as follows:

For $\varepsilon=0$ the hyperbolic fixed points of the unperturbed integrable system are smoothly connected by the invariant curves $w^{S}$ and $w^{u}$ defined before which intersect each other only at these fixed points. These invariant curves are then called separatrices because they separate the regions of the plane of section containing the various families of stable periodic orbits (resonances) of the unperturbed system. When a small perturbation is turned on $w^{S}$ and $w^{u}$ still exist but, as we have seen, they intersect each other at an infinity of homoclinic points and meander in a complicated way. They no longer deserve the name of separatrices since in the regions now bordered by them there exist infinitely many elliptic and hyperbolic points corresponding to resonances between the unperturbed system and the perturbation. As long as $\varepsilon$ is very small, the area of the region filled by the invariant curves $w^{s}$ and $w^{u}$ is also very small and can be viewed as a network of very narrow roads connecting the slightly displaced hyperbolic points of the unperturbed system. 'there is, in addition to these, an intricate network of new roads connecting the hyperbolic points created in the gaps by the perturbation but their width is so small that in most practical problems they can be ignored.

Physicists call "stochastic layers" the roads associated with the separatrices of the unperturbed system. For clarity we will call them "primary stochastic layers" while all the additional roads created by the perturbation wi.11 be called "secondary stochastic layers". 
As the size of the perturbation is further increased the primary stochastic layers widen abruptly at a certain critical value $\varepsilon_{1}$ creating regions of appreciable size where the motions are of a random nature. In the complement of these so-called stochastic regions, the invariant curves corresponding to the preserved tori still exist but they are interspersed with some of the secondary layers whose width has considerably increased but is still very small since they were initially extremely thin.

If we increase $\varepsilon$ past $\varepsilon_{1}$ nothing spectacular happens for a while until a new critical value is reached at which those secondary stochastic layers which, on some scale, were barely visible at $\varepsilon=\varepsilon_{I}$, in their turn suddenly widen at $\varepsilon=\varepsilon_{2}$, thereby creating new stochastic regions of appreciable size containing random motions.

Further increases of $\varepsilon$ beyond $\varepsilon_{2}$ will similarly result in the observation of successive widenings of more stochastic layers which will merge with the already existing wide stochastic regions at critical values, $\varepsilon_{3}$, $\varepsilon_{4}, \cdots \cdot$

An additional complication must be expected in general, that is, depending upon the region of the surface of section one is looking at, the sequence of critical values will be different.

Finally, what happens for large values of $\varepsilon$ is a matter of speculation at this time but it seems plausible that this will depend on whether the system associated with the Hamiltonian $\varepsilon H_{1}(q, p)$ describing the perturbation is integrable or not.

The determination of the critical values $\varepsilon_{1}, \varepsilon_{2}, \ldots$ and of the size. of the corresponding stochastic regions is of great importance to plasma physics, for there are already three problems known to involve stochastic 
phenomena of the kind under discussion. We have mentioned one before, namely the motion of an ion in a uniform magnetic field under the influence of an electrostatic wave. The others are the motion of an ion in a trapped-ion mode (52), and the effect of magnetic field irregularities in plasma confining devices which may cause the destruction of some or all magnetic surfaces depending on the size of the irregularities $(S-10)$. The methods of analysis under discussion are applicable to the latter problem because the divergence-free "character of the magnetic field implies that; for instance in a tokamak, the field lines induce a mapping which has the property of conserving the integral $\int \mathrm{B}$.dS where dS is the area element of any meridian plane of section $\left(54\right.$; cf also $13, n^{\circ} 305$, Vol. III). Consequently, all the pathological phenomena occuring in area-preserving mappings must also happen when a perturbation is applied to a well behaved configuration of nested toroidal magnetic surfaces. Note that in general the mapping is not area-preserving in the usual sense contrary to a common belief. This problem could be analyzed using the techniques developed for two-degree of freedom Hamiltonian systems since Hamiltonians can be constructed whose orbits are the field lines of a given magnetic field $(66,67)$. There is presently no satisfactory analytical method for the calculation of the sequence of critical values $\varepsilon_{1}, \varepsilon_{2}, \ldots$ for the simple reason that stochasticity still lacks a precise mathematical characterization. In view of the importance of the problem let us review briefly the state of our knowledge in this particular context.

As indicated before it is known that random motions are present in the neighborhood of any homoclinic point and that the Poincare map possesses an invariant hyperbolic set there. Since homoclinic points are associated with hyperbolic fixed points: it is believed that stochastic motions are due to the 
presence of a dense set of hyperbolic fixed points which are often viewed as scattering centers. According to Smale (68) this is no more than a conjecture at the present time (see also 69, p. 76). Yet this belief is supported by the following observation made in many numerical experiments: in a stochastic region orbits very close initially depart from each other at a rate which, on the average, is exponential while in the rest of the plane of section the rate of departure is only linear. To illustrate what we mean by "on the average" we reproduce in Figs. (8) and (9) graphs of the variation versus time of the distance between two adjacent orbits $(70)$. It is evident that the rate of change of this distance varies widely over each oscillation and that according to the case it is only its average which displays an exponential or linear growth.

Motivated by this empirical evidence, several authors have attempted to determine the onset of stochasticity by associating it with a change in the behavior of the distance between adjacent orbits.

For instance Mo (71) considers the distance between the origin in phase-space and a point on an orbit as a random variable obeying a generalized Langevin equation that she derives using Zwanzig's projection operator technique. From this equation she computes certain frequencies which would be the basic real frequencies in the case of an integrable system. It turns out that, as the size of the perturbation increases, one of these frequencies becomes pure imaginary for a value of $\varepsilon$ close to the one obtained via numerical integration of three different well studied systems*. The whole approach, however, lacks

*Mo actualiy uses the energy ae the parameter controlling the degree of stochasticity which, for the systems she considers, is equivalent to using $\varepsilon$. 

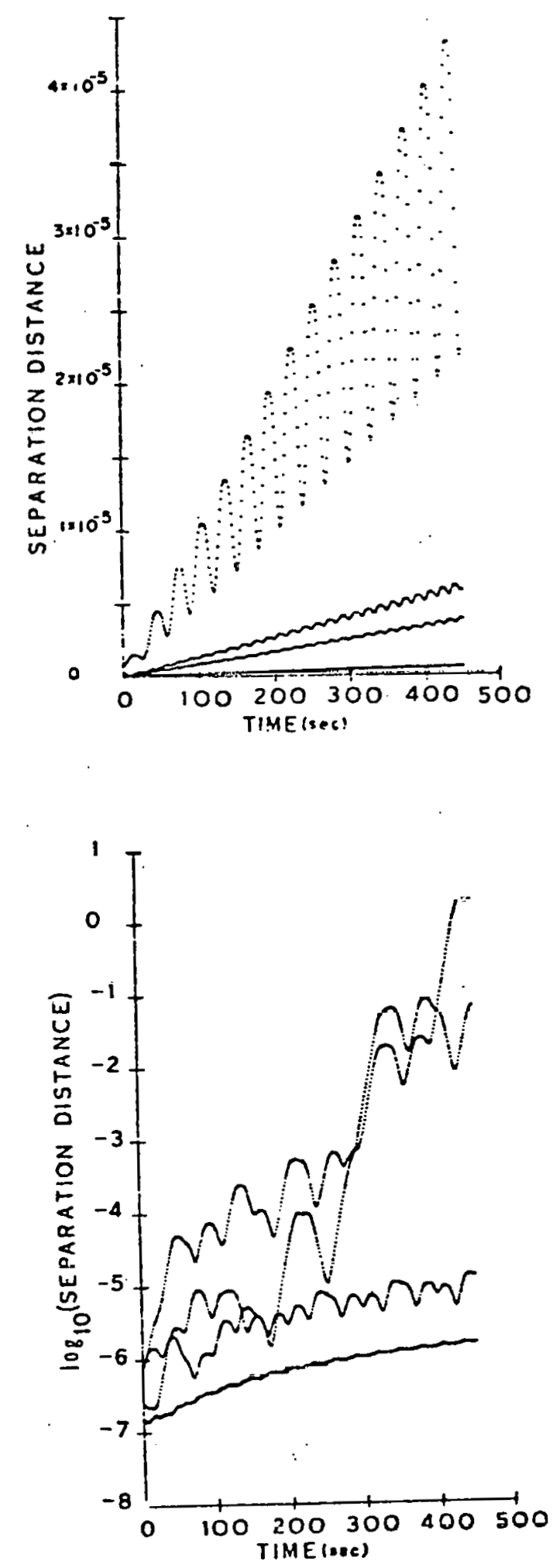

This figure sbows tie grouth of separation distane between two trajectories initially surted about $10^{-1}$ apart lo toe full phase space. Separation distance versus time is plotted for four distinct oxbit-patrs. Each orbit-pair starts in a smooth level-curve region and the linear gronth of separation distance with time is appareot.

\section{Fig. 8}

This Ilgure plots $\log _{10}$ of the aeparation distance versus time for four distinct orbtt-pairs. The generally laver-lying curves are for orbit-palrs startod fn a smooth leyel-curve regloo, and their gederally negative currature indicates the linear groath of separation dietanco with time. The Eenerally upper lyting curves are for orbit-pairs started in a stocbastic regiog of the level-curve planc, and the rapid exponential-order growth of separation distance with the is relatively clear.

$$
\text { Fig. } 9
$$


rigorous mathematical justification, and it is not clear how it can be applied to the case where the $\varepsilon$ for which stochasticity sets in depends on the region of phase-space:

Also Brumer and Duff (72) have proposed that the onset: of stochasticity can be determined from the behavior of the solutions of the so-called variational equation associated with the Hamiltonian system. Recall that the variational equation of a vector differential equation $d x / d t=f(x)$ essentially describes the behavior of the distance $n$ between two solutions $x_{1}(t)$ and $x_{2}(t)$ which are infinitesimally close at $t=0$. The vector form of the variational equation for $\eta$ is $\dot{n}=A(t) \eta$ where $A(t)$ is the Jacobian matrix of $f$ evaluated at $x=x_{1}(t)$. Brumer and Duff then argue that if the matrix $A$ possesses eigenvalues with positive real parts in a region of phase-space then the orbits will depart exponentially and stochasticity will be present. They have applied this line of reasoning to the Hamiltonians studied by Henon and Heiles (30) and by Barbanis (43) and obtained estimates which agree well with numerical results. However, although their criterion is probably necessary for the presence of stochasticity, it is certainly not sufficient since Rod has constucted integrable Hamiltonians for which the matrix A has positive eigenvalues (73). Note also that the positiveness of the real part of an eigenvalue implies only a local exponential departure of the orbits whereas, as indicated before, it is the average of the distance between orbits over relatively large times which shows an exponential increase in stochastic regions.

Another avenue for determining the onset of stochasticity is based on the notion of system entropy introduced by Kolmogorov which seems somehow related to the degree of stochasticity and therefore can perhaps be used in this connection (74). 
A third possibility is through the use of the notion of overlapping of resonances $(75,76)$. For systems with two degrees of freedom it corresponds to the observation that at $\varepsilon=\varepsilon_{1}$ the primary stochastic layers which are the first to widen as $\varepsilon$ increases seem to merge together on their facing boundaries and eventually overlap. The notion has been used successfully in many instances but it requires a difficult determination of the location of the separatrices and does not seem to be applicable to higher dimensional systems.

To sum up, there is no satisfying method available for the determination of the onset of wide spread stochasticity in a given region of phase-space. Among the various open questions related to this problem the following seem particularly important:

1) how does the number of periodic points per unit area of the plane of section vary as one of the frequencies tends to zero, i.e. as one approaches a separatrix?

2) are the fixed points in a given region elliptic or rather hyperbolic and, if so, with or without reflection?

3) is the conjecture true that as the perturbation increases in size elliptic points become hyperbolic with reflection as suggested by numerical experiments $(60)$ ?

To answer these questions when the perturbation is not small is certainly very difficult. But when the perturbation is small a powerful method is available for the determination of the periodic solutions which applies to a wide class of Hamiltonian systems. A modern version of a method invented by Foincare $(13$, chap. III, vol. 1), the method thal we have in aiml wab luvelopel by a number of mathematicians notably Cesari, Hale, and Gambill, and will 
therefore be called the PCHG method. As Poincare's method it reduces the determination of the periodic solutions to an:algebraic problem; i.e. each periodic solution is associated to a particular root of a set of transcendental equations called the determining equations. But it differs from Poincare's in the way the periodic solutions are calculated: Poincare represents them by series while in the PCHG method they are obtained as the limit of an infinite sequence of functional iterations. Since the PCHG method does not seem to be known to many physicists we feel it worthwhile to present it heuristically in this report. It will be found in Appendix $c$ together with indications for its application to slightly perturbed integrable Hamiltonian systems.

We now turn to the question of stability of the solutions of Hamiltonian systems which is intimately related to the preceding:

\subsection{Stability in the Large of Hamiltonian Systems}

For slightly perturbed integrable systems the question of the stability in the large of their solutions is settled for the case of two degrees of freedom under the conditions of the KAM theorem. Indeed in the three-dimensional phase-space corresponding to a given value of the energy, each orbit remains enclosed between adjacent tori and is therefore bounded. But for more than two degrees of freedom this is very likely no longer true in general since Arnold has shown on a particular example that there are orbits connecting neighborhoods of distant tori' (77, see also 11 and 78). A discussion of this phenomenon called "Arnold's diffusion" is presented in a forthcoming paper by Chirikov (76).

1.5 Non-canonical Perturbations of Hamiltonian Systems Because real systems'are never exactly conservative it is important to study their behavior under a small, non-canonical perturbation. The: work 
of Murdock (79-82) on such systems is particularly interesting and does not seem too well known to physicists. One of his typical results is the following $(79,81)$

Consider a sequence of pendulums each coupled to the next by a weak nonlinear spring, slightly damped, and with the first pendulum in the sequence subject to weak forcing. Then, except for solutions having amplitudes near zero, the steady state solutions are periodic with period a small integer multiple of the forcing period and with the frequencies of adjacent pendulums having small integer ratios.

These results may become important in wave-wave interactions in weak turbulence, many mode coupling, and multiple resonance.

Another recent result which also seems interesting in this connection is that of Hale and Taboas (83). They consider the equation

$$
\ddot{x}+g(x)=-\lambda \dot{x}+\mu f(t)
$$

in a neighborhood of $x=\dot{x}=0$ where $x g(x)>0, g(0)=0$, and $f$ is $2 \pi$-periodic. The equation $\ddot{x}+g(x)=0$ has a family of periodic solutions with frequelliy $\omega(a)$ which depends upon the "amplitude" a. Assume that there is a $=a_{0}$. such that $\omega\left(a_{0}\right)=1, \omega^{\prime}\left(a_{0}\right) \neq 0$, and denote by $\Gamma$ the corresponding orbit. Then the $2 \pi$-periodic solutions of $\mathrm{Eq}$. (17) which lie in a neighborhood of $\Gamma$ for $(\lambda, \mu)$ in a small neighborhood of $(0,0)$ are not contiñúous in general at $(\lambda, \mu)-0$. only when $\lambda / \mu$ tends to a constant as $(\lambda, \mu)$ tends to zero are these solutions continuous in $\lambda, \mu$. It is found that a neighborhood of the origin in the $\lambda, \mu-$ plane is divided into a finite and even number of sectors bound by curves intersecting at the origin, such that the number of $2 \pi$-periodic solutions changes by two as the point $(\lambda, \mu)$ crosses any one of these curves. The number of 
sectors and the number of such solutions within each of them depend upon the number of roots of a certain transcendental equation.

An important consequence of the theorem for the applications is that an attempt at finding these $2 \pi$-periodic solutions by classical perturbation procedures will fail, unless one takes account of the peculiar properties just described, and limits oneself to values of $\lambda, \mu$ lying within one of the sectors with $\lambda / \mu$ tending to a constant as these parameters tend to zero simultaneously.

\subsection{Method of Averaging}

The method of averaging is widely used for the study of differential systems which depend periodically on some of the variables. The aim is to simplify the system by averaging out its dependence on these variables when their rate of change is much larger than that of the others. The method is powerful and can yield valid and useful results in many instances. However, it does not always work even for relatively simple problems and it must therefore be used with some caution. We feel that the attention of the physicists has not been sufficiently drawn to the pitfalls of the method and want therefore to give examples borrowed from Arnold (84); see also 11, p. 102) which show that the solution obtained via the averaging method may have little to do with the actual solution.

The first equations considered by Arnold are

$$
\dot{\Phi}_{1}=I_{1}, \dot{\Phi}_{2}=I_{2}, \dot{I}_{1}=\varepsilon, \dot{I}_{2}=\varepsilon a \cos \left(\Phi_{1}-\Phi_{2}\right)
$$

where $\mathrm{a}>1$ and its averaged version is

$$
\dot{\mathrm{J}}_{1}=\varepsilon, \dot{\mathrm{j}}_{2}=0 \text {. }
$$


For the initial conditions

$$
I_{1}=I_{2}=J_{1}=J_{2}=1, \Phi_{1}=0, \Phi_{2}=\cos ^{-1}(1 / a)
$$

the solution is

$$
\begin{gathered}
I_{1}(t)=I_{2}(t)=1+\varepsilon t \\
J_{1}(t)=1+\varepsilon t, \quad J_{2}(t)=1 .
\end{gathered}
$$

One usually expects that the solution of the averaged system will differ from the actual solution by $\sim \varepsilon$ over an interval $O(1 / \varepsilon)$. But in the present case

$$
|I(1 / \varepsilon)-J(I / \varepsilon)|=1 \text {, }
$$

i.e. the averaged motion loses any relation to the actual motion.

The other example given by Arnold shows the effect of the passage through a resonance. The system is

$$
\dot{\Phi}_{1}=I_{1}+I_{2}, \dot{\Phi}_{2}=I_{2}, \dot{I}_{1}=\varepsilon, \dot{I}_{2}=\varepsilon \cos \left(\Phi_{1}-\Phi_{2}\right)
$$

with averaged equations

$$
\dot{\mathrm{j}}_{1}=\varepsilon, \dot{\mathrm{J}}_{2}=0
$$

For the initial conditions

$$
\Phi_{1}(0)=\Phi_{2}(0)=I_{1}(0)=0, I_{2}(0)=1
$$

which correspond to resonance, it is easily found that

$$
|I(t)-J(t)|=\left|I_{2}(t)-1\right|=\sqrt{2 \varepsilon} \int_{0}^{\tau} \operatorname{sns}\left(x^{2}\right) d x
$$

where $\tau=t \sqrt{\varepsilon / 2} \quad$. For $t=1 / \varepsilon$

$$
|I(I / \varepsilon)-J(I / \varepsilon)| \simeq C \sqrt{\varepsilon}
$$


where the constant $C$ does not depend on $\varepsilon$, which shows that the error is not of the order of $\varepsilon$ over $t \sim 1 / \varepsilon$. The effect of the resonance is to disperse the bundle of trajectories which initially differ only in their phases: The scattering of $I_{2}$ after going through the resonance is $O(\sqrt{\varepsilon})$ :

Arnold has also considered the system (84)

$$
\begin{gathered}
\dot{\Phi}=\omega(I)+\varepsilon f(I, \Phi) \\
\dot{I}=, \varepsilon F(I, \Phi)
\end{gathered}
$$

where $\Phi, \omega$, and $f$ are $k$-dimensional and $I, F$ are $\ell$-dimensional. The averaged system is

$$
\dot{\mathrm{J}}=\varepsilon \dot{\bar{F}}(\mathrm{~J})
$$

where

$$
\bar{F}(J)=(2 \pi)^{-k} \int F(J, \Phi) d \Phi
$$

When $k=2$, i.e. $\Phi=\left(\Phi_{1}, \Phi_{2}\right), \omega=\left(\omega_{1}, \omega_{2}\right)$, he has obtained the following result: if the quantity

$$
A(I, \Phi)=\left(\frac{\partial \omega_{1}}{\partial I}, F\right) \omega_{2}-\left(\frac{\partial \omega_{2}}{\partial I}, F\right) \omega_{1}
$$

does not vanish then

$$
|I(t)-J(t)|<c \sqrt{\varepsilon}(\log (1 / \varepsilon))^{2} \text { for all } 0<t<1 / \varepsilon \text {. }
$$

Note that the condition $A \neq 0$ is violated by example (18) because then $A=I_{2}-I_{1}$ a $\cos \left(\Phi_{1}-\Phi_{2}\right)$ changes $\operatorname{sign}$ at $I_{1}=I_{2}$ if a $>1$ as it was assumed; hence the difference in. the estimates of the error.

Finally, it must be borne in mind that the effects of the passage through resonance have not been rigorously studied for more than two degrees of freedom and; consequently, that there is no guarantee that the error will still be of order $\sqrt{\varepsilon}$ over $t \sim 1 / \varepsilon$ : 
For a Hamiltonian system $k=\ell=n$ (the number of degrees of freedom) in Eqs (20) which now are of the: form

$$
\begin{gathered}
\dot{\Phi} \doteq \omega(I)+\varepsilon \frac{\partial \dot{H}_{1}}{\partial I}- \\
\dot{I}=-\varepsilon \frac{\partial H_{1}}{\partial \Phi} .
\end{gathered}
$$

where $\mathrm{H}=\mathrm{H}_{\mathrm{O}}+\varepsilon \mathrm{H}_{1}, \omega=\frac{\partial \mathrm{H}_{\mathrm{O}}}{\partial \mathrm{I}}$.

The situation is now entirely different since $\bar{F}(J) \equiv 0$ so that

$J=$ constant. This is consistent with the conclusions of the KAM theorem, since the conservation of quasi-periodic motions under perturbation implies that $|I(t)-J(t)|<K$, a constant, for all times, for all initial conditions in the non-degenerate case $\left(\Delta_{1}\right.$ or $\left.\Delta_{2} \neq 0\right)$. of two degrees of freedom, and for most initial conditions for $n>2$.

If $\mathrm{H}_{\mathrm{O}}$ depends upon fewer actions than degrees of freedom, i.e. $H_{0}=H_{0}\left(I_{1}, \ldots, I_{k}\right), k<n$, (the case of so-called proper degeneracy) and if the averaged system is either integrable or close to an integrable one then there exist quasi-periodic solutions corresponding to the unperturbed system (85; see also 11,12$)$. These quasi-periodic solutions have $k$ "fast" frequencies $O$ (1) coming from the unperturbed system and $(n-k)$ "slow" frequencies $O(\varepsilon)$ that arise from the averaged system.

But, in the general case, when the averaged system is neither integrable or close to an integrable one, the relation between the solutions of the perturbed and averaged systems is unknown even over a time interval $0(1 / \varepsilon)$. From the examples and resuits given above it should be clear that the method of averaging must be used with great care since the conditions under which the actual error is of the order suggested by intuitive arguments are not well known. The lack of information regarding this question is due to 
the fact that estimates of the error usually are extremely difficult to obtain. Good sources about these matters are Hale's books (86) and (87) where the averaging method is discussed at length. 
2. Chaotic Motions in Dissipative Systems: The Case of strong Turbulence.

The study of dissipative dynamical systems has taken a new direction since the discovery about ten years ago of a new class of mathematical entities which have come to be known as strange attractors. Anticipating somewhat we can say that a strange attractor is a subset of asymptotically stable orbits in which the motions are highly sensitive to the initial conditions and display a chaotic behavior. It must be emphasized that the chaotic character of such motions is intrinsic to the model and does not require any additional assumption about the occurence of randomness. Strange attractors possess a property which is most appealing to physicists: they are structurally stable by which is meant, roughly, that their orbits are not qualitatively affected by small perturbations. The possibility of occurence of strange attractors in certain well known mathematical models is of great importance for it implies that the long term behavior of the solutions is of an apparently random nature, a feature quite unexpected in our conventional view of deterministic systems. Also there are indications that they provide better explanations for the observed random characteristics of the corresponding natural phenomena thereby validating, at least qualitatively, some mathematical models.

Strange attractors have already been found in a classic MHD problem, namely the origin of the reversals of the earth's magnetic field (88), and in a model for the continuous mode laser where they are believed to be responsible for the so-called spike instability (89). Such an attractor is also known to be present in a model for the convective instability of an atmospheric layer heated from below (90) which suggests that the turbulent phenomena that we observe in fluids are linked to the presence of strange attractors in the 
infinite-dimensional phase-space of the Navier-stokes equations. This is particularly relevant to plasma physics since Dupree has shown: (91) that the two-dimensional Navier-stokes equation is identical to the drift approximation of the vlasov equation for $k_{\prime \prime}=0$ and $k_{\perp} a_{i} \ll I\left(k_{\prime \prime}\right.$ and $k_{\perp}$ are wave numbers parallel and perpendicular to a constant magnetic field, $a_{i}$ is the ion gyroradius). Also, because strange attractors do not exist in linear systems, any theory of strong plasma turbulence based on the linearized version of the MHD equations is bound to fail if, as seems by now very likely, strange attractors are present in the phase-space of the full equations. These new considerations strongly reinforce Montgomery's arguments against the common practice of throwing away the non-linear terms (92).

We shall therefore attempt in this section to acquaint the reader with the contemporary work of various mathematicians on the problem of turbulence. Our task is not easy because of the advanced and abstract character of the mathematical methods involved. We hope that without getting bogged down in technical details our review describes with sufficient clarity the gist of the approach to the problem of turbulence taken by these mathematicians. To begin with, the Navier-stokes equations are adopted as a model for the motion of an incompressible viscous fluid. This is certainly a valid choice, at least for Reynolds numbers not too high, since the hypothesis used in deriving these equations as well satisfied by the turbulent flows that we observe. In vector form these equations are

$$
\begin{aligned}
\frac{\partial \mathrm{u}}{\partial \mathrm{t}}+(\mathrm{u} \cdot \nabla) \mathrm{u} & =-\frac{1}{\rho} \nabla \mathrm{p}+v \nabla^{2} \mathrm{u}+\mathrm{f} \\
\nabla \cdot \mathrm{u} & =0 \\
\mathrm{u} & =0 \text { or prescribed on } \partial \Omega,
\end{aligned}
$$


where $\Omega$ is the region containing the fluid, $\partial \Omega$ its boundary; $u$ the velocity field of the fluid, $\rho$ the density, $p$ the pressure, and $f$ the external force. The parameter $v$ represents the kinematic viscosity which in dimensionless variables is simply equal to the reciprocal of the Reynolds number R, i.e. $\nu=I / R$.

Let us recall in passing the major difficulties presented by the analysis of these equations in the neighborhood of $\nu=0$, that is for large Reynolds numbers. (see for instance 93, p. 287).

First, the order of the partial differential equation for the momentum is two for $\nu \neq 0$ and one for $\nu=0$. Thus standard perturbation theory cannot give us an approximation to the solution for $\nu$ small starting from the solution of the equations with $\nu=0$ - the Euler equation.

Second, for $\nu \neq 0$ the velocity $u$ is prescribed on the boundary $\partial \Omega$ because of viscosity while for the Euler case u must be parallel to $\partial \Omega$.

The usual way of attacking the problem is through the use of space averages or correlation functions that one tries to determine from the equations without solving them. This always results in a closure problem: one obtains an infinite sequence of differential or integro-differential equations which are formally satisfied by an infinite sequence of unknown functions; but the equation of rank $\mathrm{n}$ in the sequence of equations involve functions of rank $(n+1)$ in the sequence of unknowns. Consequently, truncating the sequence at the $n$-th equation can yield an approximation of known accuracy to the unknown functions of rank up to $\mathrm{n}$ included only if some appropriate information on the behavior of the functions of rank $(n+1)$ can be obtained by some other means. One of the goals of the mathematicians is to extract just this kind 
of information from the Navier-stokes equations through a qualitative analysis of the changes in the behavior of their solutions as the Reynolds number is varied.

Before dealing with the contemporary mathematical work on turbulence we must give an account of the conventional ideas about the problem as they have been proposed and developed by Landau (94; see also 95) and Hopf $(96,97)$. Landau proposed that the sequence of events observed when the Reynolds number is increased corresponds to concomitant changes in the stability of the solution of the equations. In his picture, there exists a steady laminar solution which is stable with respect to small perturbations at low values of $R$. As $R$ is increased there is a critical value $R_{1}$ at which this steady solution becomes unstable; this results in the appearance of a time dependent and stable flow which is periodic with frequency $\omega_{1}$, say. For values of $R$ slightly above $R_{1}$ this flow can be represented by superposing on the steady flow a periodic flow with an amplitude initially small but one that will increase with $R$. As $R$ is further increased there is a second critical value $R_{2}$ at which the unsteady periodic flow itself becomes unstable; again, for $R$ slightly above $R_{2}$ the flow can be looked upon as the result of the superposition over the previous flow of a periodic flow of relatively small amplitude and frequency $\omega_{2}$ (in general $\omega_{2}$ will be incommensurable with $\omega_{1}$ ), and so on. For high Reynolds numbers the velocity field would then depend on many irrationally related frequencies and therefore would look chaotic to the observer:

The plausibility of Landau's picture was later reinforced when Hopf pointed out that the successive transitions envisaged by: Landau could be explained if 
the solutions of the fluid partial differential equations undergo successive bifurcation phenomena similar to those which can happen in ordinary differential systems depending in a certain manner on a parameter (96).

However, as it has been realized since, the Hopf-Landau picture has at least three serious defects:

1) it can be shown that it leads to a Gaussian statistics which is not observed $(98,99)$;

2) the increase in the complexity of the flow womld be gradual, whercas the turbulent regime sets in at a usually sharply defined value of the Reynolds number;

3) the ultimate turbulent state would not depend in a sensitive manner on the initial conditions while the opposite is observed.

This picture was therefore in great need of improvement but it took some time until progress in mathematics made it possible for Ruelle and Takens (s-ll) in 1971 to offer a new picture which is more flexible than Landau's in its ability to model the various stages of unsteady flows observed in nature and is free of the defects mentioned above (99). To be sure, these mathematicians' picture, like Landau's, is still no more than a guess but it is a "better educated" one because it incorporates certain newly discovered properties of differential systems which were not available to Landau. This, plus the fact that recent and more accurate experiments seem to confirm its validity, has contributed to its gaining increasing interest since it was proposed.

The rest of this section is devoted to an expose of the Ruelle-Takens picture of turbulence.

We shall begin with a presentation of two mathematical phenomena of the theory of differential equations which form the basis upon which this new picture is built. 
One is the possibility for the solutions to become trapped forever in regions of phase-space where their behavior is chaotic. These regions have fewer dimensions than the phase-space itself and possess extremely complex geometric structures. They are the strange attractors mentioned earlier.

The other phenomenon, the so-called Hopf bifurcation consists in the appearance or disappearance of a periodic solution as a parameter crosses a certain critical value.

We will need some definitions and properties related to autonomous systems of differential equations of the form

$$
\left.\dot{x}=x_{\mu}(x) \quad \dot{r}=d / d t\right)
$$

We should consider the case where $x$ is an element of a functional space and $x_{\mu}$ an operator acting on it, $\mu$ being a scalar parameter that we will later identify with the Reynolds number. But it will be sufficient for the purpose of our heuristic presentation to restrict ourselves to the much simpler case where $\mathbf{x}$ and $x_{\mu}$ are $n$-dimensional real vectors in $R^{n}(s-12)$.

We assume existence and uniqueness of a solution $x(t)=\Phi_{t}\left(x_{0}\right)$ satisfying the initial conditions $x(0)=x_{0}$, i.e. $\Phi_{0}\left(x_{0}\right)=x_{0}$. The function $\Phi_{t}(x)$ is then called the flow of equation (21). Note the group property

$$
\Phi_{s+t}(x)=\Phi_{s}\left(\Phi_{t}(x)\right)
$$

for all $x, t, s$, as long as both sidcs arc defined.

A set $A \subset R^{n}$ is said to be invariant with respect to Eq. (21) if $\Phi_{t}(x) \in A$ for all $x \in A$ and all $t \in R$. Examples of such sets are a stationary solution a for which $\Phi_{t}(a)=a$ for all $t$, a closed orbit corresponding to a $T$-periodic solution $\left(\Phi_{t+T}(x)=\Phi_{t}(x)\right.$ for all $t$ if $x$ is any point of the orbit), or, more generally; a k-dimensional manifold with $k \leq n$. 
of special importance among the various types of invariant sets are those which are called attractors. Although there is no generally accepted definition of what an attractor is, we can say loosely that an invariant set. A is an attractor if it has a neighborhood $U$ such that the distance between the point $\Phi_{t}(x)$ and $A$ tends to zero as $t$ tends to infinity for all $x \in U$. For example, let a be a stationary solution, i.e. $x_{\mu}(a)=0$, such that the Jacobian matrix $\partial x_{\mu} / \partial x$ evaluated at $a$ has all its eigenvalues in the left half plane. Then it is known that there is a neighborhood of a with the above property. Other examples of attractors are a closed orbit or a torus when they are asymptotically stable (Here and in the following we mean the surface of the torus, not the solid torus, unless otherwise specified).

All these examples of attractors have been known for a long time but they do not exhaust all the possibilities, for it has by now been established that there exist attractors whose structure is topologically much different from those of a point, a closed orbit, or a torus. The possible occurence of such attractors was first pointed out by smale (50) who gave a recipe for constructing flows possessing such an attractor from a certain class of discrete dynamical systems. The attractors of the flows thus constructed by smale possess very unusual properties which led Ruelle and Takens to give them the epithet "strange" (100). At the present time the expression "strange attractor" is used to designate any attractor in which the motions have a chaotic character. As an example of a strange attractor whose structure is relatively easy to understand we will now describe one invented by shub. We borrow from smale (103) and Bowen (69).

We begin with a description of the mapping and of its properties. Consider a solid torus $T$ whose points are referred by the coordinates $(\theta, r, s)$ where $\theta$ is the azimuth of the meridian plane containing the point, 
and $r$ and $s$ are rectangular coordinates in the meridian plane as shown in Figure 10. Let: $D(\theta)$ denote: the disc intersection of $T$ by the meridian plane. The mapping $h$ is then defined by the correspondence

$$
(\theta, r, s) \stackrel{h}{\rightarrow}\left(2 \theta, \varepsilon_{1} \cos \theta+\varepsilon_{2} r, \varepsilon_{1} \sin \theta+\varepsilon_{2} s\right) .
$$

where $0<\varepsilon_{2}<\varepsilon_{1}<1 / 2$.

To see what the image of $T$ is under $h$ note first that the image of the particular disc $D(0)$ is smaller by a factor $\varepsilon_{2}$; indeed it is easily seen from (23) that this image is a disc centered at $r=\varepsilon_{1}, s=0$, and of radius $\varepsilon_{2}$. But there are other points in $D(0)$ which land there under $h$ : they come from the disc $D(\pi)$ and lie in the disc centered at $r=-\varepsilon_{1}, s=0$, with same radius $\varepsilon_{2}$ as shown in Figure li.

For an arbitrary angle $\theta \neq 0, \pi$, the points of $D(\theta)$ obtained through the application of $h$ come from the disc $D(\theta / 2)$ according to

$$
(\theta / 2, r, s) \stackrel{h}{\rightarrow}\left(\theta, \varepsilon_{1} \cos \frac{\theta}{2}+\varepsilon_{2} r, \varepsilon_{1} \sin \frac{\theta}{2}+\varepsilon_{2} s\right)
$$

and from the disc $D(\theta / 2+\pi)$ according to

$$
\left(\frac{\theta}{2}+\pi, r, s\right) \stackrel{h}{\rightarrow}\left(\theta ;-\varepsilon_{1} \cos \frac{\theta}{2}+\varepsilon_{2} r,-\varepsilon_{1} \sin \frac{\theta}{2}+\varepsilon_{2} s\right)
$$

Hence $D(\theta / 2)$ and $D(\theta / 2+\pi)$ are mapped onto smaller discs centered at $\left(\varepsilon_{1} \cos \frac{\theta}{2}, \varepsilon_{1} \sin \frac{\theta}{2}\right)$ and $\left(-\varepsilon_{1} \cos \frac{\theta}{2},-\varepsilon_{1} \sin \frac{\theta}{2}\right)$, respectively. Again, both discs have radius $\varepsilon_{2}$ (Figure 12 ).

It is then easily seen that the Image $h(T)$ of the torus $T$ under $h$ is $a$ solid torus contained in $T$ which wraps twice around the center hole of $T$ as sketched in Figure 13. Note that the effect of $h$ is to make the new torus longer and thinner than the original one.

Now apply $h$ a second time. The formula is $h^{2}(\theta, r, s)=$

$$
\left(4 \theta, \varepsilon_{1} \cos 2 \theta+\varepsilon_{2}\left(\varepsilon_{1} \cos \theta+\varepsilon_{2} r\right), \varepsilon_{1} \sin 2 \theta+\varepsilon_{2}\left(\varepsilon_{1} \sin \theta+\varepsilon_{2} s\right)\right) \text {. }
$$




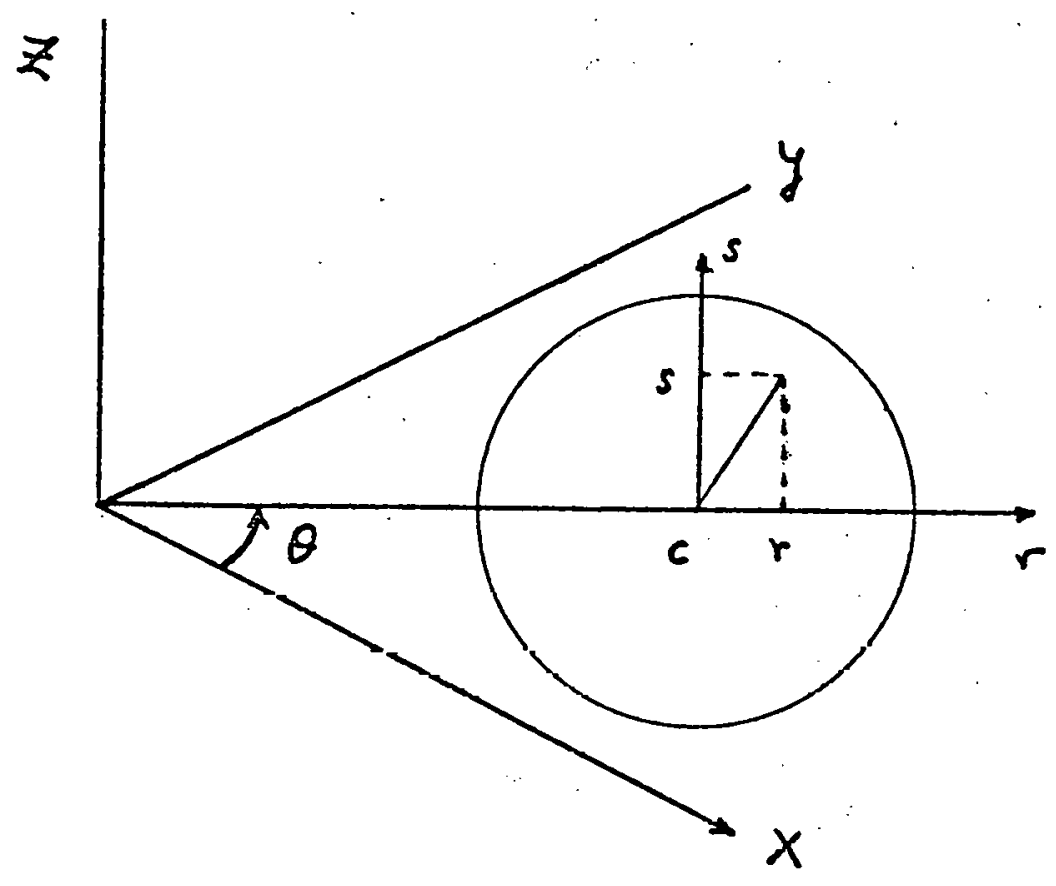

Fig. 10

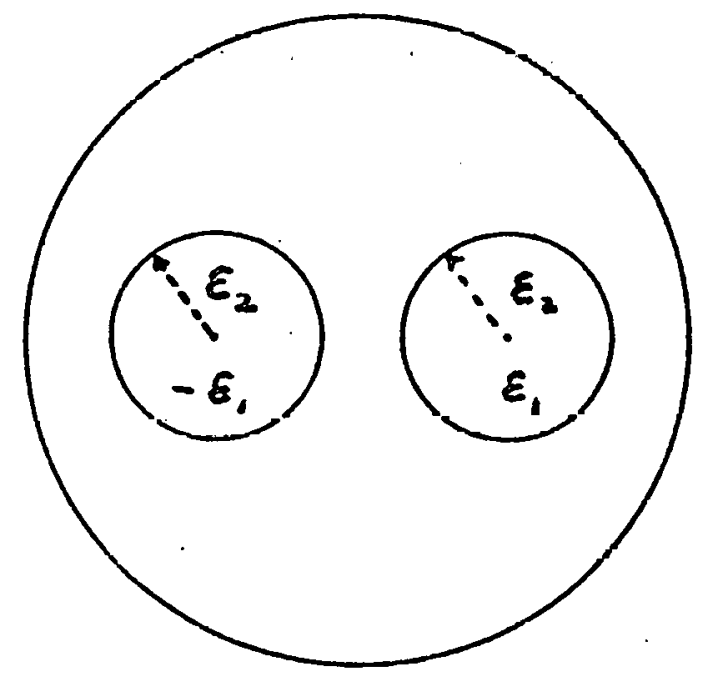

Fig. II

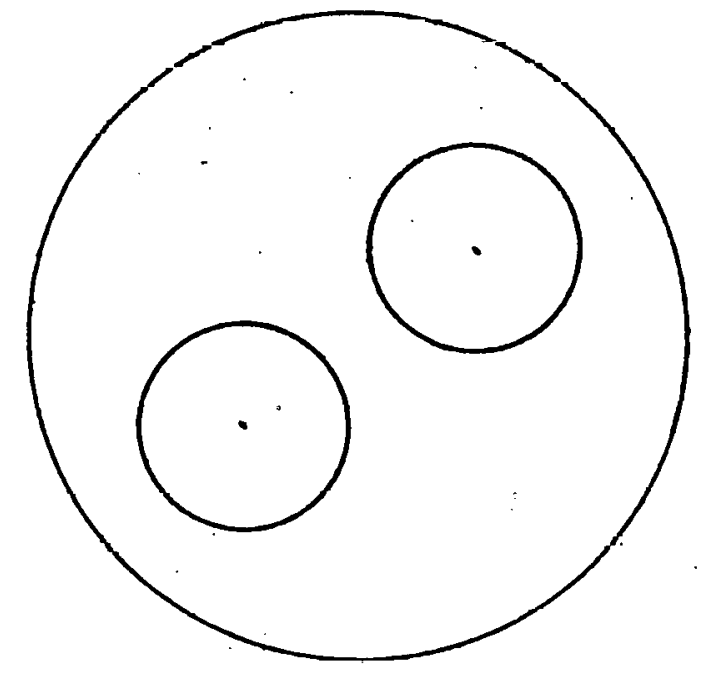

Fig. 12 

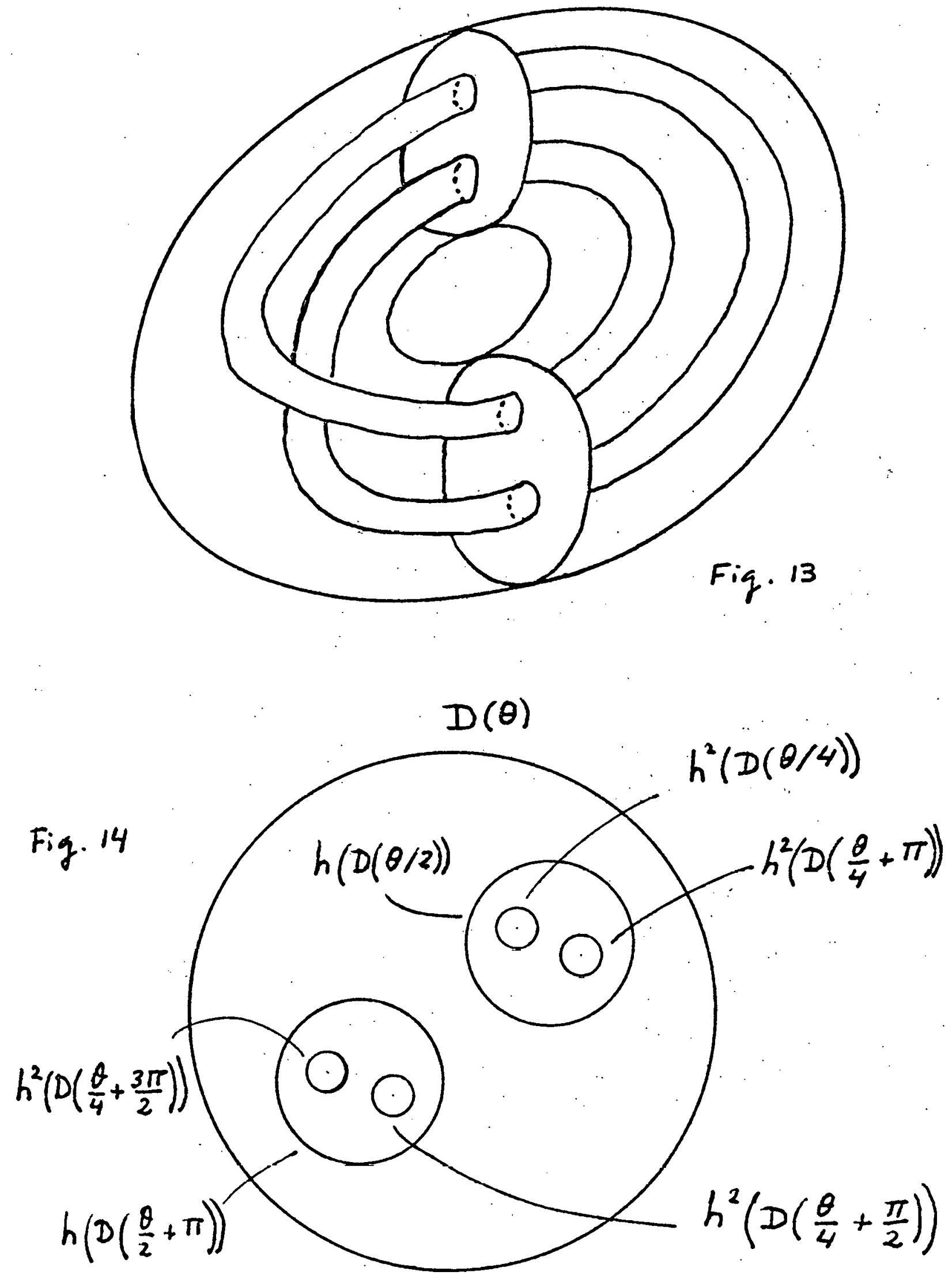
Proceeding as before it is found that

$$
h^{2}(D(\theta / 4)) \text { and } h^{2}\left(D\left(\frac{\theta}{4}+\pi\right)\right) \subset h(D(\theta / 2)) \subset D(\theta)
$$

and

$$
h^{2}\left(D\left(\frac{\theta}{4}+\frac{\pi}{2}\right) \text { and } h^{2}\left(D\left(\frac{\theta}{4}+3 \frac{\pi}{2}\right)\right) \subset h\left(D\left(\frac{\theta}{2}+\pi\right)\right) \subset D(\theta)\right.
$$

The intersection of $h^{2}(T)$ with $D(\theta)$ is thus made up of four smaller discs, two within $h(D(\theta / 2))$ and two within $h\left(D\left(\frac{\theta}{2}+\pi\right)\right)$ as shown in Figure 14. The radius of these discs is $\varepsilon_{2}^{2}$. The map $h^{2}$ therefore produces a torus which wraps four times around the central hole of $\mathrm{T}$.

clearly, if we apply the mapping $h \mathrm{n}$ times we will get a turus $\mathrm{T}_{\mathrm{n}}$ which wraps $2^{n}$ times around the hole and whose thickness $w \varepsilon_{2}^{n}$. Hence, in the limit $n \rightarrow \infty$, the set $\Lambda=\bigcap_{n>0}^{\infty} h^{n}(T)$ is a line of infinite length which winds around the hole an infinite number of times and it can be shown that the intersection of this line with any disc $D(\theta)$ is a Cantor set (see Appendix $C$ ).

Now we look for the fixed-points of $h$. In order for a point of $D(\theta)$ to be mapped into itself we must have $2 \theta=\theta+2 k \pi$ for $k=0$ or 1 . For $k=0$ we get $\theta=0$ and then the coordinates of the fixed points which are in $D(0)$ are solutions of the equations

$$
\varepsilon_{1}+c_{2} r-r \text { and } \varepsilon_{2} s=s
$$

which have the unique solution $r=\varepsilon_{1} /\left(1-\varepsilon_{2}\right), s=0$ : For $k=1$ we find another fixed point in $D(\pi)$ located at $r=-\varepsilon_{1} /\left(1-\varepsilon_{2}\right), s=0$.

In general, the $n$-th power of the mapping has one fixed point in each of the disc $D(\theta)$ such that

$$
2^{n} \theta-\theta+2 k \pi, i . \theta, \theta=2 k \pi /\left(2^{n}-1\right)
$$


where $k=0,1, \ldots, 2^{n}-2$. Hence $h^{n}$ has $2^{n}-1$ fixed points of period $n$ and in the limit of $n \rightarrow \infty$ we see that there is an infinity of fixed points of arbitrarily large period.

Among the various properties of $\mathrm{h}$ the following are essential for our purpose:

1) $\Lambda$ attracts all points of $T$ under repeated application of $h$;

2) at each point of $\Lambda$ there is a so-called "hyperbolic splitting" in the sense that $h$ expands in one direction (it makes $T$ always longer) and contracts in the other two dimensions (always shrinks the cross-section by a factor $\varepsilon_{2}$ ). 3) for the discrete dynamical system defined by $\left(\theta_{n+1}, r_{n+1}, s_{n+1}\right)=$ $h\left(\theta_{n}, r_{n}, s_{n}\right)$ the set $\Lambda$ is structurally stable.

We shall now show how a discrete dynamical system can be turned into a continuous one. The ability to effect this conversion is important because it leads to models, with known solutions, for systems such as the Navier-stokes equations whose solutions are not known in general.

Recall from section 1 that given a flow defined by some autonomous ordinary differential equations we can associate to it a discrete mapping (the Poincaré map) provided that a surface of section exists ${ }^{(*)}$. Smale's prescription for constructing a smooth flow associated to a given discrete mapping can be viewed as the reverse of a Poincare map. He has called the flow thus obtained the suspension of the given mapping. We cannot give here a description of its construction as smale did it in abstract topological terms. So, instead, we use a geometrical approach to. show first how to construct the suspension of an arbitrary mapping of the real line $f: R \rightarrow R$. This real line will be

(*) Note that the notion of surface of section can be extended to $n$-dimensional differential systems, $n$. $>3$ ' the "surface" then having dimension $n-1$. 
the $x$-axis and the flow $\Phi_{t}$ that we want to construct will be defined in the strip $B: 0 \leq y \leq 1$ of the plane of $x$ and $y$ (see Figure 15).

Clearly it is sufficient in order to define a flow to give. the prescription for constructing the unique orbit going through an arbitrary point together with the time parametrization of the motion on the orbit. This parametrization we define by the differential equations

$$
\frac{d x}{d t}=0, \frac{d y}{d t}=1
$$

The autonomous character of these equations guarantees that the flow will have the group propert.y (2.2).

Now, since $x$ must be constant according to the first equation we define the orbit through a point $\left(x_{0}, y_{0}\right) \in B$ with $y_{0}<1$ as the line $x=x_{0}$. For $t>0$, starting from $\left(x_{0}, y_{0}\right)$ the point representing the motion moves with unit speed until $y=1$. We then make it jump in zero time to the point $\left(f\left(x_{0}\right), 0\right)$; from there the orbit is the line $x=f\left(x_{0}\right)$ until $y=1$ again. The point jumps in zero time to $\left(f^{2}\left(x_{0}\right), 0\right)$, moves up along $x=f^{2}\left(x_{0}\right)$, and so on. Finally, for $t<0$ we proceed in the same fashion using the iterates $\mathrm{f}^{-\mathrm{n}}\left(\mathrm{x}_{\mathrm{o}}\right), \mathrm{n}=1,2, \cdots$.

We have thus defined the suspension flow of $f$ whose poincare map on the line of section $y=0$ is nothing but $f$ as required. Note that the fixed points of $\mathrm{f}^{\mathrm{n}}$ give rise to closed orbits on which the motion is $\mathrm{n}$-periodic.

The generalization of the above construction to a mapping $f: R^{n} \rightarrow R^{n}, n>1$, is straightforward. Let $B$ denote the subset of $R^{n+1}$ defined by the inequality $0 \leq x_{n+1} \leq 1$. The suspension is then defined on $B$ by the differential equationo

$$
\dot{x}_{i}=0, l \leq i \leq n, \quad \dot{x}_{n+1}=1
$$




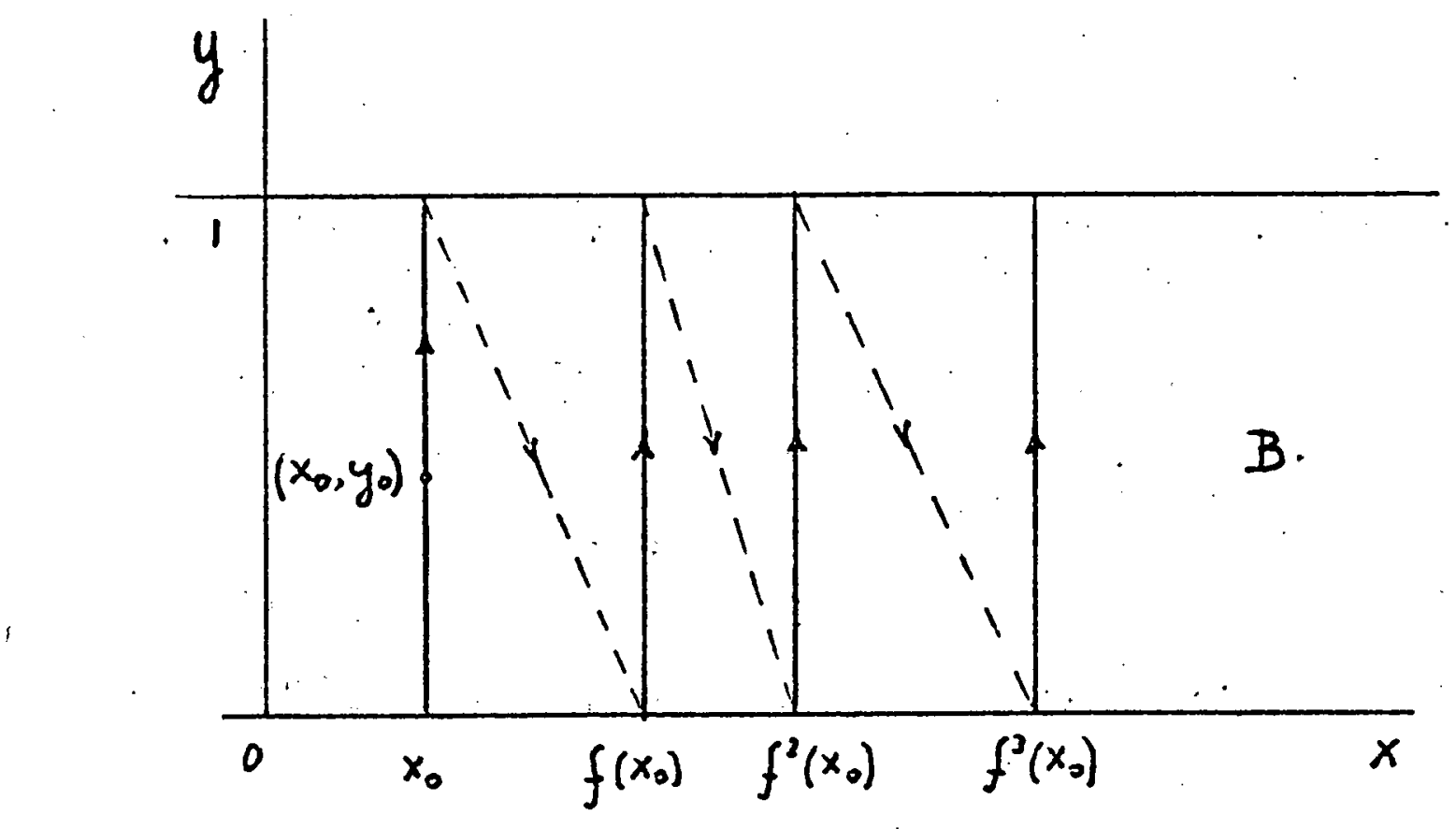

Fig. 15

$-63-$ 
The orbit through the initial point $x^{0} \in B$ is the line $x_{i}=x_{i}^{0}, 1 \leq i \leq n$, and the solution point moves on it with unit speed until $x_{n+1}=1$. It then jumps in zero time to the point $x_{i}=f_{i}\left(x^{0}\right), l \leq i \leq n, x_{n+1}=0$, moves uniformly on the line $x_{i}=f_{i}\left(x^{0}\right), l \leq i \leq n$, until $x_{n+1}=1$, jumps to $f_{i}^{2}\left(x^{0}\right), 1 \leq i \leq n, x_{n+1}=0$, and so on.

We can now apply the foregoing prescription, starting from Shub's mapping of a torus described before, to construct a flow with continuous vector field defined on $R^{4}$ for which the set $\Lambda$ is a globally stable, strange attractor as $t+\infty$.

The continuous dynamical system built this way has an extremely complicated behavior because of the hyperbolic splitting mentioned before. Indeed, consider solutions starting at $t=0$ from two points $P_{0}$ and $P_{0}^{\prime}$ a distance $\varepsilon$ apart. However small $\varepsilon$ is the distance between $\Phi_{t}\left(P_{0}\right)$ and $\Phi_{t}\left(P_{0}^{\prime}\right)$ will increase exponentially with time (excluding the case where $P_{0}$ and $P_{0}^{\prime}$ are in the same disc $D(\theta)$ ) and the transverse motion in the $r$ and $s$ coordinates will be completely different for the two orbits.

Much work has been devoted to the study of strange attractors since their relatively recent discovery and more is known about them than we can possibly cover in this report. We only mention that the ergodic properties of some of them have been investigated by several authors (see for instance 69).

We now turn to the other essential element of the Ruelie-Takens picture of turbulence, namely the Hopf bifurcation phenomenon.

- 2.1 Hopf Bifurcation Phenomenon

The llopf bifurcallun deals with the gialitative changes in behavior of the solutions of a differential equation

$$
\dot{x}=x_{\mu}(x)
$$

as the parameter $\mu$ goes through certain critical values: 
We assume as before that $x$ and $x_{\mu}$ are finite, $n$-dimensional vectors. We also assume that $x=0$ is a stationary solution, $i \cdot e: x_{\mu}(0)=0$.

Now let $A=\partial x_{\mu} / \partial x \quad$ denote the Jacobian matrix of $x_{\mu}$ evaluated at 0 and suppose there exists an interval $(a, b)$ such that for $a<\mu<b$ A has a pair of simple complex eigenvalues $\lambda(\mu)=\alpha(\mu)+i \omega(\mu)$ and $\bar{\lambda}(\mu)$ whose imaginary part does not vanish in $(a, b)$ while the real part $\alpha$ is strictly increasing with $\mu$ and vanishes for $\mu=\mu_{1} \epsilon(a, b)$. The other eigenvalues are supposed to stay in the left half plane for all $\mu$ in that interval.

It then turns out that, under mild smootheness conditions on $x_{\mu}$ the following necessarily happens (93) : as $\mu$ crosses the value $\mu_{1}$ a single periodic solution appears in the neighborhood of the origin $\mathbf{x}=0$. More precisely: if a certain function $U$ of the first, second, and third partial derivatives of $X_{\mu}(0)$ is positive the periodic solution appears for $\mu>\mu_{1}$ and is stable; as $\mu \rightarrow \mu_{1}$ Erom above, the corresponding orbit shrinks into the origin. If, instead, $U$ is negative the periodic solution exists for $\mu<\mu_{1}$, is unstable, and its orbit shrinks into 0 as $\mu \rightarrow \mu_{1}$ from below. Moreover, in both cases, as $\mu \rightarrow \mu_{1}$ the frequency tends to $\left|\omega\left(\mu_{1}\right)\right|$ and the radius of the periodic orbit approaches zero as $\sqrt{\left|\mu-\mu_{1}\right|}$.

This is Hopf bifurcation theorem (96; see also 93 for a modern account and generalizations). When $U$ is positive one says that the stationary solution undergoes a normal Hopf bifurcation whereas for $U<0$ the phenomenon is called an inverted Hopf bifurcation. Note that a more complicated behavior can occur if $U=0$ or/and if more eigenvalues cross the imaginary axis at $\mu^{\prime} \mu_{1} \cdot$

A remarkable feature of the Hopf theorem is that when its conditions are met no matter how large the (finite) dimension of the differential system is, 
we can predict: the appearance of a periodic solution and determine its stability with great ease*.

An Important extension of Hopf's theorem: was provided by Ruelle and Takens (100) who showed that, under suitable technical conditions, a stable invariant two-dimensional torus appears in the vicinity of a closed orbit when the latter becomes unstable as the parameter is increased (see also 93).

Finally, very recently; Chenciner and Iooss have given sufficient conditions which guarantee that, as an invariant two-dimensional torus becomes unstable, a stable invariant three-dimensional torus will appear (104).

We are now in the position to present the Ruelle-Takens picture.

\subsection{The Ruelle-Takens Picture of Turbulence}

'linese authors view the Navier-stokes equations as an evolution vector differential equation

$$
\frac{d u}{d t}=x_{R}(u)
$$

where $u$ is an element of the functional space $U$ of vector fields defined over the region $\Omega$ containing the fluid and satisfying the boundary conditions, $x_{R}$ is the vector field obtained through the application of the Navier-stokes operator to $u$, and $R$ is the Reynolds number.

The following assumptions are made:

1) for $1 \leq R<\bar{R}(<\infty)$ : Eq. (24) has a stationary solution $u(r, R)$ where $r$ is the position vector $(x, y, z)$ in $\Omega$;

2) the eigenvalues of the Jacobian matrix $A=\partial x_{B} / \partial u$ evaluated at $u(x, 1)$ have all strictly negative real part which implies that $u(r, 1)$ is asymptotically stable;

*The evaluation of $U$ may be cumbersome but is straightforward for it involves. only differentiations and algebraic calculations : 
as $R$ increases from 1 , successive pairs of complex eigenvalues cross the imaginary axis for $R=R_{1}, R_{2}, \ldots$ (with. $1<R_{i}<R_{i+1}$ for all $i$ ).

It follows from 3) that the stationary solution $u(r, R)$ becomes unstable and a stable closed orbit $T^{I}$ appears for $R$ slightly greater than $R_{1}$ assuming a normal Hopf bifurcation takes place. To this closed orbit corresponds a periodic flow with frequency $\omega_{1}$, say.

As $R$ increases further and reaches $R_{2}$ a second normal bifurcation occurs: the closed orbit $\mathrm{T}^{1}$ becomes unstable and there appears a stable invariant torus $\mathrm{T}^{2}$ on which the motion is quasi-periodic with frequencies $\omega_{1}, \omega_{2}$.

More generally $i t$ is assumed that as $R$ crosses $R_{k}$ from below a stable invariant torus $\mathrm{T}^{k-1}$ becomes unstable and that there appears a stable invariant torus $T^{k}$ with one more dimension.

Now, although it is possible that the motion on the torus $T^{k}, k>2$, be quasi-periodic, Ruelle and Takens conjecture that this is very unlikely, the most probable situation being the existence of strange attractors. Their conjecture is based upon their proof that the situation is indeed such on the particular torus $T^{4}$.

The novel and essential physical implication of these mathematical arguments is that the onset of turbulence can be associated with the sudden appearance of strange attractors in which the motion is intrinsically chaotic. Moreover; since the appearance of strange attractors is likely as soon as $R>R_{3}$, for $R_{1}<R<R_{3}$ the unsteady flow should display only one or two well defined frequencies; its spectrum becoming continuous in the turbulent regime associated with a strange attractor.

Finally, the possible appearance of several unconnected strange attractors with their associated basins of attraction implies that the ultimate turbulent regime may depend critically on the initial conditions. 
To sum up, strange attractors constitute new mathematical objects which should be added to the panoply of tools that can be utilized to model phenomena encountered in plasma physics.

As emphasized earlier, this picture of turbulence is no more than a guess at the present time because it relies upon many unproven assumptions. One of these is that the solutions of the Navier-stokes equations in three dimensions are globally regular, i.e. that they can be continued for all times and remain smooth. This is true in two dimensions but still a conjecture in three dimensions. Note that if strange attractors do exist the pronf for threp dimensions will probably show that solutions are globally regular provided the initial conditions lie in one of their basins of attraction (93, p. 303 ). Another assumption made by Ruelle and Takens is that the stability of the solutions can be deduced from an associated finite dimensional problem (93). Finally, Kaplan and Yorke (105) have pointed out that there is no reason to believe at present that perturbations which change the system into one with a strange attractor are more common than those which change the system into one with a stable attracting periodic orbit. This remark is important because in the Ruelle-Takens argument the non-linearities are assumed small and treated as perturbations. On the other hand, Kaplan and Yorke consider it plausible that the likelihood of strange attractors increases as the size of the nonlinearities increases.

We now indicate some experiments and analytical work which seem to confirm the validity of the Ruelle-Takens picture.

Gollub and Swinney (106) have studied the radial velocity in a fluid (water) rotating between concentric cylinders using light-scattering measurements. They have observed three distinct transitions as the Reynolds number is increased and 
each of these adds a new frequency. to the velocity spectrum. At a higher, sharply defined Reynolds number all discrete spectral peaks suddenly disappear leaving a broad hump. In a similar experiment, Swinney, Fenstermacher; and Gollub (107) have obtained the same sequence of transitions; the turbulent state however was characterized by a series of broad humps in the power spectrum.

These observations are clearly in conflict with the Landau picture but consistent with that of Ruelle and Takens.

On the analytical side, Sherman and McLaughlin (108) have studied the solutions of a model for these experiments based on the orr-Sommerfeld equation. They have found that it is possible to adjust the free parameters of the model so as to reproduce the observed dependence of the spectrum. This model also is consistent with the Ruelle-Takens picture.

Earlier attempts at solving the fluid equations had produced a chaotic attractor after a sequence of events which agree more or less with this picture (109, 110). In particular, Lorenz' work (90) deserves special consideration in view of the rather extensive investigation to which his model has been subjected by various mathematicians (s-13).

From the point of view of plasma physics this model is instructive because it illustrates the complexity arising from the competition between convective instabilities and heat transport.

\subsection{The Lorenz Model}

The problem considered by Lorenz is essentially the Benard problem of the convective motion of a fluid layer of uniform depth heated from below in a two-dimencional container. Using a method that he had proposed earlier (113), this author expanded the stream function and the temperature in Fourier series 
of space variables with time dependent coefficients, assuming free boundary conditions on the velocity field and no heat flow through the wall of the container. Substitution of these series in the partial differential equations of the problem yields an infinite set of ordinary differential equations among which Lorenz retained only three. Although no mathematical justification has been given for this severe mutilation, it is believed that the main features of the solutions to the fluid equations are qualitatively preserved, at least within some range of values of the parameters. He thus obtained the dimensionless system

$$
\begin{aligned}
& \dot{x}=-\sigma x+\sigma y \\
& \dot{y}=r x-y-x z \\
& \dot{z}=x y-b z,
\end{aligned}
$$

where $x$ is the intensity of the convective motion, $y$ is the temperature difference between the ascending and descending currents, and $z$ is the distortion of the vertical temperature profile from linearity; $\sigma$ is the Prandtl number, $b$ is a constant related to the basic spatial frequency in the $x$ direction, and $r$ is the Rayleigh number.

Lorenz found through numerical integration that, in spite of its relative simplicity, this system displays a remarkable variety in its behavior the most interesting phenomenon being the appearance, for $r$ large enough, of an attractor which is neither a point nor a closed orbit.

Among the properties of the Lorenz system the following are elementary and easy to verify:

1) the system is invariant under the transformation $x \rightarrow-x, y \rightarrow-y, z \rightarrow z$. This is a consequence of the invariance of the equations of motion under a reflection through a vertical line at the center of the container. 
2). the divergence of the vector whose components are the righthand sides of the equations has a constant value $-(\sigma+b+1)$. Consequently, the corresponding flow shrinks volumes in the phase-space at a uniform rate which; for parameter values of interest $(\sigma=10, b=8 / 3)$ is -13.66 and therefore is quite large; a unit volume is transformed into the volume exp $(-132 / 3) \simeq 10^{-6}$ over a unit interval of time.

3) all solutions are boundedas $t \rightarrow \infty$. In fact one can show that there exists a ball $B$ in phase-space such that all solution curves eventually get trapped inside it for ever. The shrinking properties of the flow then implies that the volume of the image of this ball tends to zero at $t \rightarrow \infty$ and all solutions tend to a set which is closed and has measure zero.

Since the ultimate behavior of all solutions depends upon the nature of this set we shall review how its structure changes as $r$ is increased from zero, keeping the other parameters fixed as before $(\sigma=10, b=8 / 3)$.

1) $0 \leq x \leq 1$ : the origin $x=y=z=0$ is then the only singular point and is globally attracting; physically, it corresponds to conduction: the fluid layer is at rest and the temperature varies linearly between the two surfaces:

2) $r>1$ : there are now three singular points: the origin which has become unstable and the two points

$$
c=(\sqrt{b(r-1)}, \sqrt{b(r-1)}, r-1), C^{\prime}=(-\sqrt{b(r-1)},-\sqrt{b(r-1)}, r-1)
$$

which correspond to steady convection.

For all $r>1$ there is a two-dimensional manifold (a surface) containing the origin which is invariant under the flow and on which the solution curves 
approach the origin as $t \rightarrow \infty$. There is also a one-dimensional invariant manifold (a curve) containing 0 along which the point is reached as $t \rightarrow-\infty$.

The points. $C$ and $C^{\prime}$ are locally stable for $I<r \leq r_{2}=470 / 19 \simeq 24.74$. For $r>r_{2}$ they become unstable because two complex conjugate: eignvalues move from the left half plane to the right one. The corresponding Hopf bifurcation is of the inverted type; i.e. in the neighborhood of each of these points there exists an unstable periodic orbit for $r$ slightly smaller than $r_{2}$, whose size shrinks to zero as $r \rightarrow r_{2}$ from below.

There are two important transition values for $r$ which have been found numerically:

At $r=r_{0} \simeq 13.926$ the unstable trajectories of the origin return to 0 as $t \rightarrow \infty$ (they are homoclinic orbits in the terminology of section 1 ). For $1<r<r_{0}$ all trajectories tend to one of the rest points $0, C$, or $C^{\prime}$, while for $r>r_{0}$ there are infinitely many periodic orbits and infinitely many unstable "turbulent orbits" (105) which do not tend to any point or periodic orbit. The set of these orbits has zero measure.

At $r=r_{1} \simeq 24.06$ each of the unstable trajectories from 0 tend to an unstable periodic orbit as $t \rightarrow \infty$. These two periodic orbits are of the sadהle type regarding stability, i.e. a Poincare map of their neighborhood gives rise to a hyperbolic point whose stable manifold contains the corresponding trajectory. coming from the origin.

For $x>24.06$ there is a chaotic stable attractor which probably disappears at some value of $r$ between 28 and 50 since it was not observed in Lorenz' numerical integrations for $r=50$.

We now describe in more detail what happens in the intervals $\left(r_{0}, r_{1}\right)$ and $\left(r_{1}, r_{2}\right)$ successively $(105)$ 
1) $r_{0}<r<r_{1}$ : the stationary points $C$ and $C^{\prime}$ have:neighborhoods $N_{C}$ and $\mathrm{N}_{\mathrm{C}^{\prime}}$. such that the trajectory starting from any point in $\mathrm{N}_{\mathrm{C}}$ (resp: $\mathbf{N}_{\mathrm{C}^{\prime}}$ ) tends to $C$ (resp. $C^{\prime}$ ) as $t \rightarrow \infty$. Because two eigenvalues are complex at $C^{\prime}$ and $C^{\prime}$, such trajectory approaches its limit point like a spiral so that $x, y, z$, display damped harmonic oscillations about their limiting values.

If the initial point is far from $C$ and ' $'$ ', the trajectory first oscillates a number of times between two regions located one in $x>0$ the other in $x<0$ until it becomes trapped either in $\mathrm{N}_{c}$ or in $\mathrm{N}_{c^{\prime}}$. On the average, the number of such oscillations is larger the closer $r$ is to $r_{1}$ and seems to tend to infinity as $r \rightarrow r_{1}$. Also this number of wide oscillations critically depends upon the initial point so that it is impossible to predict how many more oscillations are going to occur from the knowledge that so many have already taken place. The corresponding solutions look chaotic for some time which may be very long if $r$ is only slightly smaller than $r_{1}$ and then rapidly settle down at $C$ or $C^{\prime}$ after few damped harmonic oscillations about it. Kaplan and Yorke call such behavior metastable chaos and suggest that it may be what creveling et al. (114) have observed in the flow of a fluid through pipes: more than one hundred chaotic oscillations took place before the oscillations become regular and damp out.

2) $r_{1}<r<r_{3}$ : $C$ and $C^{\prime}$ are still locally stable but there is a chaotic stable attractor which attracts for ever trajectories coming from a set of positive measure.

We will now give an idea of the behavior of the orbits in the strange attractor for $r=28>r_{2}$. The points $C$ and $C^{\prime}$ are then unstable like the origin. At each point there is a manifold (a surface) $w_{c, c}^{\mathrm{u}}$. made up of orbits 
which spiral away from the point and, transverse to this manifold, there is a stable manifold $w_{c, c}^{S}$ ! (a curve) along which the point is approached as $t \rightarrow \infty$. The situation is sketched in Figure 16.

The overall behavior of an orbit in the strange attractor is then as follows: 'supposing that we start from a point close to the origin the orbit first gets close to $c$, say, and then, moving very close to $w_{c}^{u}$ spirals away from $c$. In the course of its.motion around the line $w_{c}^{s}$ it minimum distance from the origin decreases at each revolution until, suddenly, it goes towards the other singular point $C^{\prime}$. It then executes a certain number of revolutinns around the line $\mathrm{w}_{\mathrm{C}^{\prime}}^{\mathrm{s}}$, spiralling away from $\mathrm{C}^{\prime}$, then goes back to the vicinity . of $C_{r}$ splrals away from it., ete. A remarkablc feature of the inution is that the number of consecutive. revolutions about $C$ or $C^{\prime}$ varies widely in a manner that is unpredictable in practice. This can be explained with the help of Figure 17 in which, for clarity, we have not drawn. the manifolds $w_{c, c}^{u}$, and $w_{c, c}^{s}$ of $C$ and $C^{\prime}$. In a neighborhood of the origin the strange attractor (which is a surface) is made up of uncountably many shept.s, all sontaining the unstable manifold $w_{0}^{\mathrm{u}}$ (a line) of the origin, which intersect transverse arcs in Cantor sets. This geometrical object looks locally like a book of infinitely many pages which are bound along the line $w_{0}^{u}$. We show three of these pages labeled 1, 2, and 3. On each page the configuration of the nrhits is hyporbolio; on page 1 , for instance, there is only one orbit $s_{1} 0$ going to 0 which is the intersection of this page with the stable manifold $w_{0}^{3}$ (a surface).

we are now going to trace two different orbits labeled $\alpha$ and $\beta$ respectively, which start from points of the segment $s_{1} b_{1}$ on page 1 . First $\alpha$ leaves the vicinity of $0_{\text {and }}$ is attracted by the stable manifold $w_{0}^{s}$ of $c$. It gets close to the surface $w_{c}^{u}$ and after one ravolution returns close to the origin where it moves on page 2. Because we have chosen to make it cross the segment 


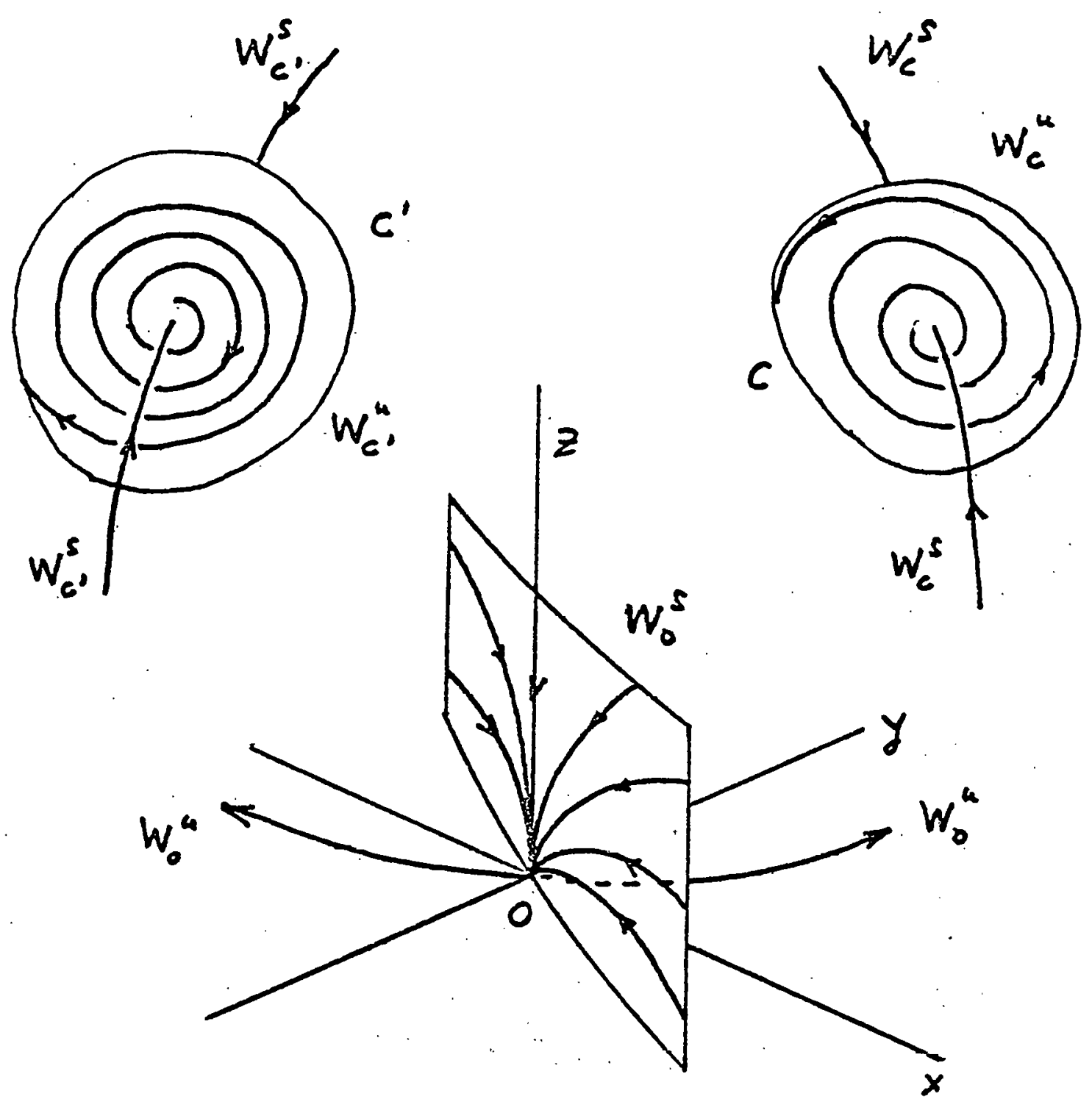

Fig. 16

$-75-$ 


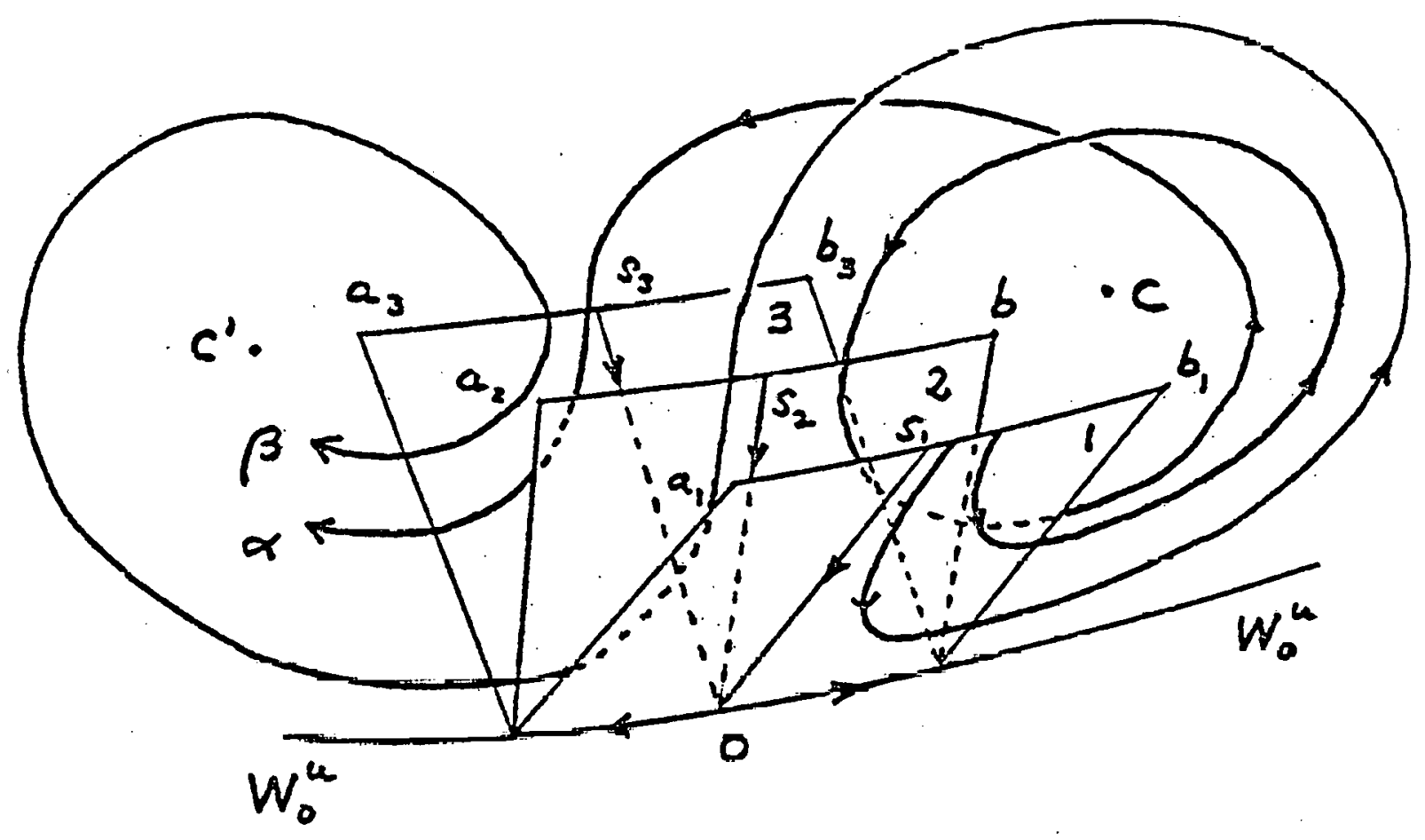

Fig. 17 
$a_{2} b_{2}$ to the right of $s_{2}$ its next revolution again takes place around $c$. However when it comes back towards 0 it lands on the segment $a_{3} b_{3}$ of page 3 at a point to the left of $\mathrm{S}_{3}$; as a result its next revolution will be around $\mathrm{C}^{\prime}$.

We now look at the orbit $\beta$ which also leaves the vicinity of 0 on page 1 but from a point of $s_{1} b_{1}$ closer to $s_{1}$. It winds around $c$ like $\alpha$ initially but after a single revolution it intersects $a_{2} b_{2}$ at a point to the left of $s_{2}$; as a result, it goes around $C^{\prime}$ and will revolve at least twice around it since we have chosen to make it intersect the segment $a_{3} b_{3}$ of page 3 to the left of $s_{3}$. The picture is then clear: the strange attractor has an infinity of hyperbolic points at the origin, each located in one of its sheets and these points are responsible, by their peculiar nature, for the chaotic behavior of the orbits in the strange attractor.

The reader might wonder how it is possible for an orbit to go from the vicinity of $C$ to that of $C^{\prime}$ since the stable manifold $w_{0}^{S}$ seems to be in the way. Actually it is not but its shape is so complicated to sketch that we have given up showing it in the figure.

Finally, we must caution the reader that we designed Figure 17 for the sole purpose of illustrating the mechanism of chaos in the strange attractor: the topological inconsistencies that it may contain are of no importance in this connection.

The preceding outline of the properties of the Lorenz system covers most of what is known at the present time. For more details we refer the reader to the orlginal reports.

It is to be expected that much further research will be devoted to the study of the Lorenz eystem in spite of its questionable value as a valid approximation to the fluid equations. The reason for this is that the system 
is probably one of the simplest dissipative systems that can display the kind of chaotic behavior believed to be at the roots of an explanation of turbulence. Another reason for a continuing interest in this system is that it is also encountered in laser physics where it is believed to explain the onset of self-pulsing ( $(89)$

Among the various questions being investigated one is whether the motion in the Lorenz attractor is ergodic in a sense consistent with the dissipative structure of the system. This is of central importance to physics in general and to plasma physics in particular since the notion of "anomalous" transport coefficients like that of thermal conductivity of the Benard convective layer has a meaning only if it can be shown that for all motions in the attractor the asymptotic value of time averages does not depend upon initial conditions. An outline of a possible approach to this problem has been given by Lanford (102) 


\section{Appendix A}

\section{Superconvergent Perturbation Method}

In the perturbation method described in the text the size of the perturbation decreases by a factor $\varepsilon$ at each stage of approximation. But this rate of decrease is too small to overcome the difficulties due to the presence of the small denominators. Fortunately; the perturbation scheme can be modified so that the $n$-th approximation is of order $\varepsilon^{2^{n}}$, the fast convergence of the resulting series then compensating for the divergence related to the small denominators. This scheme due to Kolmogorov (15) is essentially equivalent to Newton's method of successive approximations to the root of a scalar equation $f(x)=0$. More precisely, since the objects to be approximated are functions rather than numbers, it is Kantorovitch's generalization (115) of Newton's method to equations in function space that was proposed by Kolmogorov and used by Arnold in his proof of the KAM theorem.

We shall outline the principal features of the method for the case of a Hamiltonian of the form

$$
H(p, q)=H_{0}(p)+\varepsilon H_{1}(p, q)+\varepsilon^{2} H_{2}(p, q)+\ldots
$$

where $H_{k}(p, q)$ is $2 \pi$-periodic in each of the $q^{\prime} s$ for all $k^{\prime} s$.

Re-write this as

$$
H(p, q)=H_{0}(p)+\varepsilon \bar{H}_{1}(p)+\varepsilon \bar{H}_{1}(p, q)+\varepsilon^{2} H_{2}(p, q)+\ldots
$$

where for an arbitrary function $f(p, q) 2 \pi$-periodic in the $q^{\prime} s$

$$
\bar{f}(p)=\frac{1}{(2 \pi)^{n}} \int f(p, q) d q
$$

and $\bar{f}(p, q)=f(p, q)-\bar{f}(p)$. Hence $f(p, q)$ has mean value zeru. 
Apply the canonical transformation generated by the function $p^{\prime} q+\varepsilon S_{1}\left(p^{\prime}, q\right)$ where $s_{1}$ is to be determined so that in the new Hamiltonian the perturbation is $o\left(\varepsilon^{2}\right)$. From the Hamilton-Jacobi theory the old momentum $p$ is given by

$$
p=p^{\prime}+\varepsilon \frac{\partial S_{1}}{\partial q}
$$

Replace $\mathrm{p}$ by this expression in $\mathrm{H}$ and expand about $\mathrm{p}=\mathrm{p}^{\prime}$ obtaining

$$
\begin{aligned}
& H\left(p^{\prime}, q\right)=H_{o}\left(p^{\prime}\right)+\varepsilon \frac{\partial H_{o}}{\partial p_{i}^{\prime}} \frac{\partial S_{1}}{\partial q_{i}}+\frac{\varepsilon^{2}}{2 !} \frac{\partial^{2} H_{o}}{\partial p_{i}^{\prime} \partial p_{j}^{\prime}} \frac{\partial s_{1}}{\partial q_{i}} \frac{\partial s_{1}}{\partial q_{j}}+\ldots \\
& +\varepsilon \overline{\mathrm{H}}_{1}\left(\mathrm{p}^{\prime}\right)+\varepsilon^{2} \frac{\partial \overline{\mathrm{H}}_{1}}{\partial \mathrm{p}_{i}^{\prime}} \frac{\partial \mathrm{S}_{1}}{\partial \mathrm{q}_{i}}+\ldots \\
& +\varepsilon{\widetilde{H_{1}}}_{1}\left(p^{\prime}, q\right)+\varepsilon^{2} \frac{\partial \widetilde{H_{1}}}{\partial p_{i}^{\prime}} \frac{\partial S_{1}}{\partial q_{i}}+\ldots \\
& +\varepsilon^{2} \mathrm{H}_{2}\left(\mathrm{p}^{\prime}, \mathrm{q}\right)+\ldots
\end{aligned}
$$

where summation over repeated indices is implied and the omitted terms are of order $\varepsilon^{3}$.

In order to make the $q$-dependent terms in $\varepsilon$ disappear, choose $s_{1}$ such that

$$
\omega_{i}^{(i)}\left(p^{\prime}\right) \frac{\partial S_{1}}{\partial q_{i}}+\mathbb{F}_{1}\left(p^{\prime}, q\right)=0
$$

where $\omega_{i}^{(1)}\left(p^{\prime}\right)=\partial H_{0} / \partial p_{i}^{\prime}$.

Then solve Eq. (Al) toqether with the equation

$$
\mathrm{q}^{\prime}=\mathrm{q}+\varepsilon \frac{\partial \mathrm{S}_{1}}{\partial \mathrm{p}^{!}}
$$

for $q$ in terms of $\mathrm{p}^{\prime}, \mathrm{q}^{\prime}$ and substitute the resulting expressions into Eq. (A2). This can be done using the generalization of Lagrange's formula to vector equations (115). 
Drop the superscripts on $p^{\prime}$ and $q^{\prime}$ and write the new Hamiltonian as

$$
\begin{aligned}
H(p, q) & =H_{0}(p)+\varepsilon \bar{H}_{1}^{(1)}(p)+\varepsilon^{2} \bar{H}_{3}(2)(p)+\varepsilon^{3} H_{3}^{(2)}(p) \\
& +\varepsilon^{2} \widetilde{H}_{2}(2)(p, q)+\varepsilon^{3} \widetilde{H}_{3}(2)(p, q)+o\left(\varepsilon^{4}\right)
\end{aligned}
$$

Now apply a canonical transformation generated by $p^{\prime} q+\varepsilon^{2} s_{2}\left(p^{\prime}, q\right)$. Substitution of

$$
p=p^{\prime}+\varepsilon^{2} \frac{\partial s_{2}}{\partial q}
$$

into $\mathrm{H}$ and expansion about $\mathrm{p}=\mathrm{p}^{\prime}$ yields

$$
\begin{aligned}
H\left(p^{\prime}, q\right) & =H_{0}\left(p^{\prime}\right)+\varepsilon^{2} \frac{\partial H_{o}}{\partial p_{i}^{\prime}} \frac{\partial S_{2}}{\partial q_{i}}+\varepsilon^{4} \ldots \\
& +\varepsilon \bar{H}_{I}^{(1)}\left(p^{\prime}\right)+\varepsilon^{3} \frac{\partial \bar{H}_{I}^{(1)}}{\partial p_{i}^{\prime}} \frac{\partial S_{2}}{\partial q_{i}}+\varepsilon^{5} \ldots \\
& +\varepsilon^{2} \bar{H}_{2}^{(2)}\left(p^{\prime}\right)+\varepsilon^{4} \ldots \\
& +\varepsilon^{3} \bar{H}_{3}^{(2)}\left(p^{\prime}\right)+\varepsilon^{5} \ldots \\
& +\varepsilon^{2} \widetilde{H}_{2}^{(2)}\left(p^{\prime}, q\right)+\varepsilon^{4} \ldots \\
& +\varepsilon^{3} \widetilde{H}_{3}^{(2)}\left(p^{\prime}, q\right)+\varepsilon^{5} \ldots \\
& +\ldots
\end{aligned}
$$

It is readily seen that not only the terms in $\varepsilon^{2}$ but also those in $\varepsilon^{3}$ can be eliminated provided $S_{2}$ satisfies the equation

$$
\left(\frac{\partial \mathrm{H}_{0}}{\partial p_{i}^{\prime}}+\varepsilon \frac{\partial \bar{H}_{1}}{\partial p_{i}^{\prime}}\right) \frac{\partial S_{2}}{\partial q_{i}}+\widetilde{H}_{2}^{(2)}\left(p^{\prime}, q\right)+\varepsilon \widetilde{H}_{3}^{(2)}\left(p^{\prime}, q\right)=0 .
$$

It can be shown by induction that the procedure may be continued indefinitely; i.e. the n-th Hamiltonian will contain a perturbation of order $\varepsilon^{\nu}$ with: $v=2^{\text {n. }}$. 
Notes: 1) Howland has very recently discussed this method and indicated how it can be implemented without having to use Lagrange's inversion formula through the use of Lie transforms (117).

2) Bogoliubov (118) and Bogoliubov et al. (119) have extended the method to non-Hamiltonian systems. A translation of their work does not seem to be available at this time.

Remarks: As illustrated by its success in the proof of the KAM theorem the superconvergent method is certainly a powerful tool in pure analysis. However, as pointed out by Cary (120), it is not evident at this time that the computational labor that it requires for a given order of approximation is less than when using traditional methods. On the other hand, a symbol manipulation program such as MACSYMA (121) may reduce the labor sufficiently to make this approach more useful (122). 


\section{Note: on the: KAM. Theorem}

In the main text, for the simplicity of the presentation, we have considered Hamiltonians in which the smallness of the perturbation is due to: the presence of a small factor $\varepsilon$ in the perturbing part. Actually, in his proof of the KAM theorem, Arnold only assumes that

$$
H(p, q)=H_{0}(p)+H_{1}(p, q)
$$

with $\mathrm{H}_{1}$ bounded in a compact region of the phase-space. Denoting the bound by $M$, he proves the theorem for $M$ sufficiently small.

Regarding the conditions (10) of the text, i.e.

$$
\Delta_{1}=\operatorname{det}\left(\frac{\partial^{2} \mathrm{H}_{0}}{\partial p_{i} \partial \mathrm{p}_{j}}\right) \neq 0 \text { or } \quad \Delta_{2}=\operatorname{det}\left(\begin{array}{cc}
\frac{\partial^{2} \mathrm{H}_{0}}{\partial p_{i} \partial p_{j}} & \frac{\partial \mathrm{H}_{o}}{\partial p_{i}} \\
\frac{\partial \mathrm{H}_{0}}{\partial \mathrm{p}_{j}} & 0
\end{array}\right) \neq 0
$$

note that they are not equivalent each corresponding to a different version of the theorem; but each of them is sufficient for the validity of the conclusions. (See for instance 11, p. 269). 
Periodic solutions of a slightly perturbed integrable Hamiltonian system by

the PCHG method.

1 - The PCHG Method:

Designed for the determination of the periodic solutions of differential equations, the method takes various forms depending on the nature of the equations of interest. We will concentrate on the case where the equations can be written in vector form as

$$
\dot{x}=\mu q(t, \mu, \mu)
$$

for, as we shall show, the equations of a slightly perturbed integrable system can be broughe tó this form by an appropriate change of variables. 'In (Cl) $x$ and $q$ are $n$-vectors, $q$ is $T$-periodic in $t$ and $\mu$ is a small parameter. For $\mu=0$ the solution is $x(t)=a_{0}$, a constant vector, and the goal is to find for $\mu$ small the $T$-periodic solutions, if any, which reduce to constant vectors as $\mu \rightarrow 0$.

The method rests upon four basic theorems (cf ref. 86, theorems 6-1 to 6-4, Chap. 6) whose contents, leaving aside technical details, are as follows:

Given an n-dimensional constant vector a there exists for $\mu$ sufficiently small a unique vector function $x^{*}(t, a, \mu)$ with the following properties: a) $x *$ is continuous and $T$-periodic in $t$, and its mean value is precisely equal to the given vector $a, i . e$.

$$
\frac{1}{\underline{T}} \int_{0}^{T} x^{\star}(t, a, \mu) d t=a
$$

b) it satisfies the differential equation

$$
\dot{x}^{*}=\mu q\left(t, x^{*} ; \mu\right)-\mu \frac{1}{T} \int_{0}^{T} q\left(t, x^{*}(t, a, \mu), \mu\right) d t
$$


c) $x^{\star}(t, a, 0)=a$, i.e. for $\mu=0 x^{\star}$ reduces to the given constant vector. Moreover the function $x^{*}$ is the limit as $k$ tends to infinity of the sequence of functions defined recursively by

$$
\begin{aligned}
& \ddot{x}^{(0)}(t)=a \\
& x^{(k+1)}(t)=a+\mu \int^{t} d \xi\left\{q\left(\xi, x^{(k)}(\xi, a, \mu), \mu\right)\right. \\
&\left.-\frac{1}{T} \int_{0}^{T} q\left(t, x^{(k)}(t, a, \mu), \mu\right) d t\right\}
\end{aligned}
$$

which converges for $\mu$ sufficiently small and where the integral $\int^{t}$ denotes the (unique) primitive (i.e. indefinite integral) whose mean value is zero. Note that all $x^{(k)}$ are free from secular terms (i.e. of terms in $t^{m}, m=1,2, \ldots$ ) since the integrand in (C3.) also has zero mean value.

As a consequence of this theorem if there exists a vector a $(\mu)$ depending continuously on $\mu$ and such that

$$
\frac{1}{T} \int_{0}^{T} q\left(t, x^{*}(t, a(\mu), \mu), \mu\right) d t=0
$$

then $x *(t, a(\mu), \mu)$ is a T-periodic solution of Eq. (Cl) as is readily seen from Eq. (C2́)

In the limit $\mu \rightarrow 0$ we have : if there exists an $n$-vector $a(0)=a_{0}$ such that

$$
\text { (a) } q_{0}\left(a_{0}, 0\right)=0 \text { and (b) } \operatorname{det}\left(\frac{\partial q_{0}\left(a_{0}, 0\right)}{\partial x}\right) \neq 0
$$

where

$$
q_{0}(x, \mu)=\frac{1}{T} \int_{0}^{T} q(t, x, \mu) d t
$$


then there exists for $\mu$ sufficiently small a T-periodic solution $x(t, \mu)$ such that $x(t, 0):=a_{0}$

Finally; the method is exhaustive, i.e. it produces all T-periodic solutions which reduce to a constant vector as $\mu \rightarrow 0$.

The equations $q_{0}\left(a_{0}, 0\right)=0$ and more generally

$$
q_{0}(x \cdot(k)(t, a(\mu), \mu), \mu)=0
$$

are called the determining equations.

The following comments are in order at this point :

a) the periodic solutions of Eq. (Cl) necessarily have period $\mathrm{T}$ or an integer multiple of $T$ in which case they are called subharmonics. The PCHG method can be implemented so as to determine all of these solutions (123, sf alsn ref. 124, p. 399).

b) in practice it may happen that the equation $q_{0}\left(a_{0}, 0\right)=0$ is identically satisfied for all $a_{0}$; one must then look for the smallest value of $k$ such that the determining equation $q_{0}\left(x^{(k)}, \mu\right)=0$ is not an identity and thus determines the vector $a(\mu)$ (for details see ref. 123, p. 46).

$\underline{2}$ - Slightly Perturbed Integrable Hamiltonian Systems

We assume that the Hamiltonian is of the form

$$
H(J, w) \equiv H_{0}(J)+\varepsilon H_{1}(J, w, \varepsilon)
$$

where $\mathrm{J}-\mathrm{W}$ are action-angle variables and $\mathrm{H}_{1}$ is $2 \pi$-periodic in $\mathrm{w}$. The differential system is

$$
\begin{aligned}
& \dot{\omega}=\omega(\mathrm{J})+\varepsilon \frac{\partial \mathrm{H}_{1}}{\partial \mathrm{J}} \\
& \dot{\mathrm{J}}=-\varepsilon \frac{\partial \mathrm{H}_{1}}{\partial w} .
\end{aligned} \quad\left(\omega(\mathrm{J})=\frac{\partial \mathrm{H}_{\mathrm{o}}}{\partial \mathrm{J}}\right)
$$


For $\varepsilon=0$ the solution is

$$
\begin{aligned}
& w(t)=w\left(J^{\circ}\right) t+w^{\circ}(\bmod 2 \pi) \\
& J(t)=J^{\circ},
\end{aligned}
$$

where $w^{\circ}, J^{\circ}$ are constants of integration.

Following Poincare $\left(13 ; n^{\circ} .42\right.$, Vol. 1) we choose a vector $\mathrm{J}^{\circ}$ such that $\omega_{i}\left(J^{\circ}\right)=m_{i} v$ where $\nu$ is the greatest common frequency and the $m^{\prime} s$ are integers. We are going to look for periodic solutions with period $T=2 \pi / \nu$ which reduce for $\varepsilon=0$ to functions $w(t)$ and $J(t)$ of the form $(c 8)$. The values of $w^{0}$ corresponding to these solutions will be determined by the determining equations of an auxiliary system.

If we define new variables and parameter $I, \phi, \mu$ by

$$
\begin{aligned}
& w=\omega\left(J^{0}\right) t+\phi \\
& J=J^{0}+\mu I \\
& \mu=\sqrt{\varepsilon}
\end{aligned}
$$

the variables $\phi$ and I obey the differential equations

$$
\begin{aligned}
& \dot{\phi}=\mu \Omega\left(J^{\circ}\right) I+\mu^{2} \frac{\partial}{\partial J} H_{1}\left(J^{\circ}+\mu I, \omega\left(J^{\circ}\right) t+\phi, \mu^{2}\right)+\mu^{2} F(I, \mu) \\
& \dot{I}=-\mu \frac{\partial}{\partial w} H_{1}\left(J^{\circ}+\mu I, \omega\left(J^{\circ}\right) t+\phi, \mu^{2}\right)
\end{aligned}
$$

where

$$
\Omega\left(J^{\circ}\right)=\frac{\partial \omega\left(J^{\circ}\right)}{\partial J}=\left(\frac{\partial^{2} H}{\partial_{1} T_{i} \partial_{j}}\right)
$$

is the Hessian matrix of $\mathrm{H}_{0}$ evaluated at $\mathrm{J}=\mathrm{J}^{\circ}$ and the function $\mathrm{F}$ is defined by

$$
\omega\left(J^{\circ}+\mu I\right)-\omega\left(J^{\circ}\right)=\mu \Omega\left(J^{\circ}\right) I+\mu^{2} F(I, \mu) .
$$


These equations are in the required form and the determining equations for the zero-th approximation $\phi^{\circ}, I^{\circ}$ (the vector $a_{0}$ of parag. I above) are

$$
\begin{aligned}
& \frac{1}{T} \int_{0}^{T} \Omega\left(J^{\circ}\right) I^{\circ} d t=\Omega\left(J^{\circ}\right) I^{\circ}=0 \\
& \frac{1}{T} \int_{0}^{T} \frac{\partial}{\partial w} H_{I}\left(J^{\circ}, \omega\left(J^{\circ}\right) t+\phi^{\circ}, 0\right) d t=0 .
\end{aligned}
$$

The vector $\mathrm{I}^{\circ}$ is uniquely determined by the linear homogenous equation (C9-a) provided that $\operatorname{det} \Omega\left(\mathrm{J}^{\circ}\right) \neq 0$ in which case $I^{0}=0$. The existence of periodic solutions then depends upon whether Eq. (C9-b) have real solutions in $\phi^{\circ}$. To caoh root $\phi^{\circ}$ of these equations corresponds a. T-periodic solution of system (C7) which for $\mu=0$ reduces to the functions given by (C8) where $w^{\circ}=\phi^{\circ}$. Note that from the form of Eq. (C8) if $\phi^{\circ}$ is a root so is $\phi^{\circ}+\omega\left(J^{\circ}\right) \tau$ where $\tau$ is an arbitrary constant.

The case where $\operatorname{det} \Omega\left(\mathrm{J}^{\circ}\right)=0$ must be handled differently:

If all the components of $J$ appear in $H_{0}(J)$ it is often possible to find an equivalent Hamiltonian for which this determinant does not vanish (13). This possibility is based on the fact that if $U(x)$ is any scalar function the Hamiltonian system corresponding to the new Hamiltonian

$$
H^{\prime}(J, W, \varepsilon, \varepsilon) \equiv U(H(J, W, \varepsilon)) / U^{\prime}(E)
$$

is easily seen to be identical to system (C7) provided the value of the energy E be appropriately chosen. The difficulty will be removed since the Hessian of $\mathrm{H}^{\prime}(J, W, E, 0)$ in general will be diffexent from that of $\mathrm{H}_{0}$. Hamiltonian (12) provides an example of such a situation for which the choice $H^{\prime}$ (J, W, E, $\varepsilon) \equiv \mathrm{H}^{2}(J, W, \varepsilon) /(2 E)$ resolves the difficulty as long as $\beta \neq 0$. When $\beta=0$ the Hamiltonian $\mathrm{H}_{0}=\mathrm{J}_{1}+\mathrm{VJ}_{2}$ represents two uncoupled harmonic oscillators and it is not necessary to chànge parameter. Indeed for an $\mathbf{n}$-degree of freedom Hamiltonian 


$$
H \equiv \cdot \sum_{i=1}^{\dot{n}} \omega_{i} J_{i}+\varepsilon H_{1}(J, w, \varepsilon)
$$

the variables $\phi$ and I defined by the equations

$$
w=\omega t+\phi, j=I
$$

obey the differential system

$$
\begin{aligned}
\dot{\phi} & =\varepsilon \frac{\partial}{\partial J} \mathrm{H}_{1}(I, \omega t+\phi, \varepsilon) \\
\dot{I} & =-\varepsilon \frac{\partial}{\partial w^{v}} H_{1}(I, \omega t+\phi, \varepsilon)
\end{aligned}
$$

which is in the required form (Cl).

$-89-$ 


\section{Cantor Sets}

As an example of a Cantor set we describe Cantor's ternary set:

To construct it mark the points $1 / 3$ and $2 / 3$ on the closed interval

$I=[0,1]$ and then delete the points of the open interval $(1 / 3,2 / 3)$ called the "middle third". Call $T_{1}$ the remainder of the points in $I$, i.e.

$$
\mathrm{T}_{1}=\left[0, \frac{1}{3}\right] \cup\left[\frac{2}{3}, 1\right] .
$$

Now trisect the segment $[0,1 / 3]$ at $1 / 9$ and $2 / 9$, and the seyment $[2 / 3,1]$ at $7 / 9$ and $8 / 9$. Then delete the middle third of each segment, i.e. the open sets $(1 / 9,2 / 9)$ and $(7 / 9,8 / 9)$. Call $\mathrm{T}_{2}$ the remainder of the points in $\mathrm{T}_{1}$, i.e.

$$
\mathrm{T}_{2}=\left[0, \frac{1}{9}\right] \cup\left[\frac{2}{9}, \frac{1}{3}\right] \cup\left[\frac{2}{3}, \frac{7}{9}\right] \cup\left[\frac{8}{9}, 1\right] .
$$

We can continue this process indefinitely obtaining a sequence of sets such that $\mathrm{T}_{1} \supset \mathrm{T}_{2} \supset \mathrm{T}_{3} \supset \ldots$ as shown:

I $\mathrm{T}_{1}$

$\mathrm{T}_{2}$
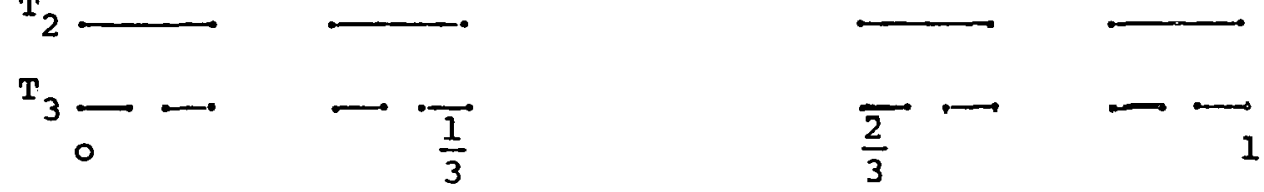

Cantor's ternary set $T$ is then defined as the intersection of all $T_{n}$ 's, i.e. $T=\prod_{n=0}^{\infty} T_{n}$ with $T_{0}=I$. It has the following properties:

clearly $T_{n}$ consists of $2^{n}$ disjoint closed intervals that we can number consecutively from left to right. We say that one of these intervals is odd or even according to the parity of its number. 
Now let $x \in T$ be a point in $T$ and define the infinite sequence of symbols $a=\left(a_{1} ; a_{2}, \ldots\right)$ by the prescription

$$
a_{n}=\left\{\begin{array}{l}
0 \text { if } x \text { belongs to an odd interval in } T_{n} \\
2 \text { if } x \text { belongs to an even interval in } T_{n}
\end{array}\right.
$$

The sequence a corresponds to the representation of the number $x$ in the system of base 3 :

$$
x=a_{1}(1 / 3)+a_{2}(1 / 9)+\cdots+a_{n}\left(1 / 3^{n}\right)+\cdots
$$

Intuitively we see therefore that all points in $T$ are in one-to-one correspondence with the infinite sequences of two symbols (here 0 and 2 ). Using the above representation it can be shown that $T$ has the power of the continuum (i.e. is non-denumerable) and yet has measure zero. Note that there are Cantor sets with positive measure (125). 
S-1 The theory of Hamiltonian systems can be found in many books on classical mechanics notably whittaker (1); Goldstein (2); Corben and stehle (3), Ter Haar (4), and Meirovitch (5). A minimum background in traditional calculus, linear algebra, elementary analysis, and a little topology is sufficient for a thorough understanding of the material presented in these books. None of these authors makes any reference to the possibility of existence of random motions. The only book written in the traditional vein that we have found where these motions are discussed at an elementary level is Bartlett's (6).

The modern theory of Hamiltonian systems is constructed within the framework of modern analysis and can be found in Abraham's text book (7). Its understanding requires familiaxity with "basic undergraduate calculus and algebra, and a limited amount of olassical analysis, point set topology, and elementary mechanics". A background in modern advanced calculus on manifolds, exterior algebra, and general topology is given which is barely - sufficient for the understanding of the new formulation of the theory. Although the book contains a translation of kolmoqorov's lecture in which he announced his thcorem on slightly perturbed Hamiltonian systems; random motions are not covered. Another modern presentation of the theory at an advanced level is sternberg's in his lectures on differential geometry which deals with random motions (8).

Finally, a very extensive coverage of Hamiltonian syetems theory both in the traditional and in the new formulation including random motions can be found in Hagihara's monumental treatise on celestial mechanics (9). 
Note added in proof: We have just received a copy of a French translation of a text book by Arnold published in Russian in 1974 and entitled "Mathematical Methods of Classical-Mechanics" where the author gives a modern, comprehensive, and self-contained presentation of the subject. For the interested reader the reference is "Méthodes mathématiques de la mécanique classique" par V. Arnold, traduit du. russe par Djilali Embarek, Traduction francaise Editions Mir, Moscou 1976.

The following new books. should also be useful:

- Differential topology with a view to applications, by D.R.J. Chillingworth, Pitman Publishing, London 1977.

- Analysis, Manifolds, and physics, by Y. Choquet-Bruhat, C. Dewitt-Morett, and M. Dillard-Bleick, Elsevier North-Holland Publishing Co., New York, 1977. - Nonlinearity and Functional Analysis, by Melvyn S. Berger, Academic Press, New York, 1977.

S-2 Arnold's theorem can be found in (7) where an outline of the proof is given. For a full proof see his original paper (10), Arnold and Avez (11), or (12).

s-3 For a modern exposition of action-angle variables see (5) or (12).

S-4 Besides the proof of Kolmogorov's theorem Arnold's paper (5) contains a clear discussion of the stability problems of classical mechanics. See also (11) and (12).

S-5 Actually Moser (17) gave an analogous result corresponding to systems of two degrees of freedom in the differentiable case. See also (18).

S-6 A comprehensive exposé of the available perturbation methods can be found in Giacaglia (19) and Nayfeh (20). See also Born (21) and Meirovitch (5). 
S-7 Many papers have appeared which deal with the problem of ion motion in a uniform magnetic field under the influence of an electrostatic wave; lists of references can be found in $(22-25)$.

S-8 In connection with this question Hénon makes the following remarks in (28) : "Recall that the transformation associated with a conservative dynamical system possesses an integral invariant, i.e. it conserves the quantity

$$
\iint_{D} I(x, y) d x d y
$$

evaluated over any domain $D, \quad x$ and $y$ are the coordinates of the surfare of section and $\mathrm{I}$ is a function which depends on the surface of section chosen. It is only for certain particular choices that $I$ is identically equal to $I$ and consequently that the transformation conserves areas in the strict sense".

s-9 There is a considerable literature on the properties of area-preserving mappings; the basic references are $(9,11,13,17,18,27,29)$. For specific area-preserving mappings, see $(6,30-43)$.

$S-10$. The random behavior of magnetic field lines was apparently first observed in numerical calculations by Gelfand et al. (53) in 1961. Discussions of the problem can be found in Morozov and solovecv (54) and Grad (55). The destruction of magnetic surfaces caused by various types of perturbations has been studied notably by Rosenbluth et al. (56), Filonenko et al. (57), Finn (58), and Finn and Kaw (59), Stix $(60,61)$, and Rechester and Stix (62) where references to other works of physicists can be found. Mathematical studies of the problem have been carrled out by Melnikov (63) who pointed out that the widely used method of averaging of Bogoliubov 
and Mitropolsky (64) may not be applicable to this problem (see his reference:\#7 in 63), and by Moser (65) who restricts himself to the region immediately surrounding the central, circular field line of a toroidal configuration in the case where curl $\mathrm{B}=0$.

S-11 The Ruelle-Takens picture of turbulence was presented in (100). See also (93) and (99).

\$-12 For an introduction to ordinary:differential equations with emphasis on their dynamical aspects see the book by Hirsch and Smale (101). See also Lanford's lecture notes (102).

S-13 Mathematical investigations of the Lorenz system have been done by Williams (111), Guckenheimer in (93), Ruelle (99), Kaplan and Yorke (105). We have: not had access to the contents of a recent paper by Afraimovitch, Bikov, : and Shilnikov (112). 
1. Whittaker, E.T.; "Analytical Dynamics of Particles and Rigld Bodies", 4th edition; Cambridge Univ. Press, 1959.

2. Goldstein, H., "Classical Mechanics"; Addison-Wesley, Reading 1950.

3. Corben, C. and P. Stehle, "Classical Mechanics", Wiley, New York 1950.

4. Ter Haar, D., "Elements of Hamiltonian Mechanics", Pergamon Press, New York 1971 .

5. Meirovitch;:L., "Methods of Analytical Mechanics"; McGraw-Hill, New York 1970.

6. Bartlett;:J.H., "Classical and Modern Mechanics", Univ. of Alabama Press, University of Alabama 1975.

7. Abraham, R. (with assistance of J.E. Marsden), "Foundations of Mechanics", Benjamin, New York 1967.

8. Sternberg, S., "Lectures on Differential Geometry", Prentice-Hall, Englewood-Cliff, New Jersey 1964.

9. Hagihara, Y., "Celestial Mechanics", MIT Press, Cambridge 1970.

10. Arnold, V.I.. A Theorem of Liouville Concerning Integrable Problems of Dynamics, Sibirsk. Mat. 2., 4 (1963) 471-474; Amer. Math. Soc. Translation, Series: 2, 61 (1964) 292-296.

11. Arnold, V.I. and A. Avez, "Ergodic Problems of Classical Mechanics", Benjamin, New York 1968.

12. Arnold, V.I., Small Denominators and Problems of Stability of Motion in Classical and Celestial Mechanics, Russian Math. Surveys, 18, 6 (1963) $85-191$.

13. Poincaré, H., "Les méthodes nouvelles de la mecanique céleste", 3 vol., Gauthier-Villars, Paris 1892-99; reprinted by Dover, New York 1957. English translation: "New Methods of Celestial Mechanics", NASA TF-450, April 1967.

14. Birkhoff, G.D., Collected mathematical papers, Amer. Math. Soc., Providence 1950; reprinted by Dover, New York 1968.

15. Kolmogorov, A.N., Preservation of Conditionally Periodic Movements with Small Change in the Hamilton Function, Dokl. Akad. Nauk SSSR, 98 (1954) 527-530, Englioh translation: Los Alamos Bcienlific Laburalusy, Lus Alannus, New Mexico, IAA-TR-71-67. 
16. Arnold, V.I., Proof of a Theorem of A.N. Kolmogorov on the Preservation of Conditionally Periodic Motions under a Small Perturbation of the Hamiltonian, Russian Math. Surveys, 18, 5 (1963) 9-36.

17. Moser, J., On Invariant Curves: of Area Preserving Mappings of an Annulus, Nachr. Akad, Wiss. Gottingen, Math. Phys. Klasse, Nr 1 (1962) 1-20.

18. Moser, J., "Stable and Random Motions in Dynamical Systems"; Princeton Univ. Press, Princeton 1973.

19. Giacaglia, G.E.O., "Perturbation Methods in Non-Linear Systems", SpringerVerlag, New York 1972.

20. Nayfeh, A., "Perturbation Methods", Wiley, New York 1973.

21. Born, M.; "The Mechanics of the Atom", translation of the original 1925 edition by J.W. Fisher and D.R. Hartree, Ungar Publ. Co., New York 1960.

22. Karney, C.F.F. and A. Bers, Stochastic Ion Heating by a Perpendicularly Propagating Electrostatic Wave, PRR 76/35-2, Res. Lab. of Electronics, MIT, March $1977 a$.

23. Karney, C.F.F., Stochastic Heating of Ions in a Tokamak by RF Power, Ph.D. Thesis, MIT, Dept. of EE \& CS, May 1977.

24. Smith, G.R. and A.N. Kaufman, Stochastic Acceleration by a Single Wave in a Magnetic Field, Phys. Rev: Lett., 35 (1975) 1613-1616.

25. Sinth, G.R., Stochastic Acceleration by a single Wave in a Magnetized Plasma, Ph.D. Thesis, Univ. of California, Berkeley 1977.

26. Niven, I., Irrational Numbers, Math. Assoc. of America 1965.

27. Birkhoff, G.D., Surface Transformations and Their Dynamical Applications, Acta Math., 43 (1920) 1-119; or in Vol. 2 of ref. 14.

28. Hénon, M., Problèmes Numeriques Liés á la Recherche des Solutions des Transformations Ponctuelles Conservatives, Colloques Internationaux du CNRS; : N ${ }^{\circ} 229$, "Transformations Ponctuelles et leurs Applications", Toulouse 10-14 Sept. 1973; CNRS Paris 1976, pp. 387-398.

29: Greene, J.M., Two-Dimensional Measure-Preserving Mappings, J. Math. Phys., g (1968) 760-768.

30. Hénon, M. and C. Heiles; The Applicability of the Third Integral of Motion: Some Numerical Experiments, Astron. J., 69 (1964) 73-79.

31. Hénon, M., Numerical Study of Quadratic Area-Preserving Mappings, Quart. Appl. Math., 27 (1969) 291-312. 
32. Hitzl, D.L., The Swinging Spring - Invariant Curves Formed by QuasiPeriodic Solutions II, Astron. \& Astrophys., 41 (1975) 197-198.

33. Helleman, R.G., on the Iterative Solutions of a stochastic Mapping, in "Statistical Mechanics and Statistical Methods, Theory and Applications", U. Landman ed., Plenum, New York. 1977.

34. Danby, J.M.A., Wild Dynamical Systems and the Role of Two or More Small Divisors, in "Periodic orbits, Stability and Resonances", G.E.O. Giacaglia ed., D. Reidel Publ. Co., Dordrecht 1970.

35. " " ", The Evolution of Periodic Orbits Close to Homoclinic Points, Celest. Mech., 8 (1973) 273-280.

36. Dragt, A.J. and J.M. Finn, Insolubility of Trapped Particle Motion in a Magnetic Dipole Field, J. Geophys. Kes., 81 (19"76) 2327-2340.

37. Brahic, A., Numerlcal Study of a Simple Dynamical System, I The Associated Plane Area-Preserving Mapping, Astron. \& Astrophys., 12, (1971) 98-110.

38. Kannou, F., Numerical Study of 'Dtscrete Plane Area-Preserving Mappings, ibid., 31 (1974) 289-301.

39. Braun, M., On the Applicability of the Third Integral of Motion, J. Diff. Eqs.: 13 (1973) 300-318.

40. Braun, M., Numerical Studies of an Area-Preserving Mapping, in "Dynamical Systems, An International Symposium", L. Cesari, J.K. Hale, and J. LaSalle eds, Vol. 2, Academic Press, New York 1976.

41. Aubry, S., on the Dynamics of Structural Phase-Transitions - Lattice Locking and Ergodic Theory, Preprint (Brookhaven National Lab., Associated Universities Inc., Upton, Long Island, NY 11973).

42. Froeschle, C., A Numerical study of the stochasticity of Dynamical Systems with Two Degrees of Freedom, Astron. \& Astrophys., 9 (1970) 15-23.

43. Barbanis, B., Trapping of Particles in a Time-Dependent Hamiltonian, Celest. Mech., 14 (1976) 201-208.

44. Moser, J., The Analytic Invariants of an Area-Preserving Mapping Near a Hyperbolic Fixed Point, Comm. Pure Axpl. Math., 9 (1956) 673-692.

45. Hadamard, J., Les surfaces à courbures opposées et leurs lignes qeodésiques, J. de Math., 4 (1898) 27-73.

46. Morse, M. and G.A. Hedlund, Symbolic Dynamics, Amer. J. Math., 60 (1938) 815-866. 
47. Smale, S.; Diffeomorphisms with Many Periodic Points, Differential and Combinatorial Topology, (A Symposium in Honor of Marston Morse), pp. 63-80, Princetón University Press, Princeton 1965.

48. Alekseev, V.M., Quasi-Random Oscillations of a One-Dimensional Oscillator, Soviet Math. Dokl., 8, 6 (1967) 1421-1424.

49. Alekseev, V.M., Quasi-Random Oscillations and Qualitative Aspects of Celestial Mechanics, 9th Summer Mathematics School, 2nd ed., revised, Yu. A. Mitropolski and A.N. Sharovsky eds, Acad. of Sci. of the Ukrainian S.S.R., Naukkova Dumka Publ. House; Kiev 1976 (Russian); English translation: ERDA-tr-302; available from Technical Information Services, Oak Ridge, Tennessee:

50. Smale, s.; Differentiable Dynamical Systems, Bull. Amer. Math. Soc., 73 (1967) 747-817.

51. Zehnder,E., Homoclinic Points Near Elliptic Fixed Points, Comm. Pure Appl. Math., 26 (1973.) 131-182.

52. Smith, G.R., Overlap of Bounce Resonances and the motion of Ions in a Trapped-Ion Mode, Phys. Rev. Lett., 38 (1977) 970-973.

53. Gelfand, I.M., N.M. Zueva, A.I. Morozov and L.S. Soloveev, Magnetic Surfaces of the Three-Path Helical Magnetic Field Excited by a Crimped Field, Soviet Physics-Tech. Phys., 6 (1962) 852-855.

54. Morozov, A.I. and L.S. Soloveev, The structure of Magnetic Fields, Rev. Plasma Phys., 2 (1966) 1-101.

55. Grad, H., Toroidal Containment of a Plasma, Phys. of Fluids, 10 (1967) 137-154.

56. Rosenbluth, M.N., R.z. Sagdeev, J.B. Taylor and G.M. Zaslavsky, Destruction of Magnetic Surfaces by Magnetic Field Irregularities, Nuclear Fusion, $\underline{6}$ (1966) 297-300.

57. Filonenko, N.N., R.z. Sagdeev and G.M. Zaslavsky, Destruction of Magnetic Surfaces by Magnetic Field Irregularities : Part II, Nuclear Fusion, 7 (1967) 253-265.

58. Finn, J.M., The Destruction of Magnetic Surfaces in Tokamaks by Current Perturbations, ibid, 15 (1975) 845-854.

59. Finn, J.M. and P.K. Kaw, Coalescence Instability of Magnetic Islands, Phys. of Fluids, 20 (1977) 72-78. 
60. Stix, T.H., Current Penetration and Plasma Disruption, Phys. Rev. Lett., 36 (1976) .521-524.

61. Stix, T.H., Aspects of Stochàstic Heating, Proc. Conf. on Plasma Heating, Varenna 1976.

62. Rechester, A.B. and T.H. Stix, Magnetic Braiding Due to Weak Asymetry, Phys. Rev. Lett., 36 (1976) 587-590.

63. Melnikov, V.K., Lines of Force of a Magnetic Field, Soviet Phys. Dokl., I (1962) .502-504.

64. Bogoliubov, N.N. and Y.A. Mitropolski, Asymptotic Methods in the Theory of Non-Linear Oscillations, Hindustan Publ. Corp., Delhi 1961.

65. Moser, J., Leetures on Hamiltonian Systems, Memulrs Araer. Math. Suc., 81. $(1968) \cdot 1-60$.

66. Kerst; D.W., The Influence of Errors on Plasma-Confining Magnetic Fields, Plasma Phys. (J. Nucl. Energy Part C), 4 (1962) 253-263.

67. Whiteman,: K.J., Invariants and stability in Classical Mechanics, Rep. Prog: Phys.; 40 (1977) 1033-1069.

68. Smale, S., private communication.

69. Bowen, R., On Axiom-A Diffeomorphisms, Lectures Given at the NSF Regional Conference held at North Dakota State Univ. (Fargo), June 1977.

70. Ford, J. and G.H. Lunsford, Stochastic Behavior of Resonant Nearly Linear Oscillator Systems in the Limit of zero Non-Linear Coupling, Phys. Rev: $A, \underline{1}(1970)$ 59-70.

71. Mo, K.C., Theoretical Prediction for the onset of Widespread Instability in Conservative Nonlinear Oscillator Systems, Physica, 57 (1972) 445-454.

72. Brumer, P. and J.W. Duff, A Variational Equation Approach to the onset of Statistical Intramolecular Energy Transfer, J. of Chem. Phys., 65 (1976) 3566-3574.

73. Ford, J., private communication.

74. Benettin, G., L. Galgani, and J.M. Strelcyn, Kolmogorov Entropy and Numerical Experiments, Phys. Rev. A, 14, 6 (1976) 2338-2345.

75. Zaslavskii, G.M., and B.V. Chirikov, Stochastic Instability of Non-1inear Oscillations, Soviet Phys. Uspekhi, 14, 5 (1972) 545-672.

76. Chirikov, B.V., I Univereal Instability of Many-Dimenoional Ocoillator Systems, proc. of the 1977 Como Conference on Stochastic Behavior in Classical and Quantum Hamiltonian Systems, G. Casati and J. Ford eds, to appear in Phys. Reports. 
77. Arnold, V.I., Instability of Dymamical Systems with Several Degrees of Freedom, Soviet Math. Dokl., 5 (1964) 581-585.

78. Arnold; V.I., The Stability Problem and Ergodic Properties for classical Systems, proc. Internat. Cong. Math. (Moscow 1966) 387-392; Izdat. "Mir", Moscow 1968 (Russian); Amer: Math. Soc. Transl. (2) 70 (1968) 5-11.

79. Murdock; J., Resonance Capture in Certain Nearly Hamiltonian Systems, J. Diff. Eqs., 17 (1975) 361-374.

80. Murdock, J., Nearly Hamiltonian Systems in Two Degrees of Freedom, Int. J. Non-Linear Mech., 10 (1975) 259-270.

81. Murdock, J., Nearly Hamiltonlan Systems in Non Linear Mechanics : Averaging and Energy Methods, Indiana Univ. Math. J., 25 (1976) 499-523.

82. Murdock,.J., Global Results by Local Averaging for Nearly Hamiltonian Systems, in "Dynamical Systems: An International Symposium", L. Cesari, J.K.: Hale, and J. LaSalle eds, Vol 2, pp. 24-27, Academic Press, New York 1976.

83. Hale, J.K. and P.Z. Taboas, Interaction of Damping and Forcing in a Second order Equation, Preprint.

84. Arnold, V.I., Conditions for the Applicability, and Estimate of the Error, of an Averaging Method for Systems Which Pass Through states of Resonance in the Course of Their Evolution, Soviet Math. Dokl., $\underline{6}$ (1965) 331-334.

85. Arnold, V.I., On the classical Theory of Perturbations and the Problem of Stability of planetary systems, ibid., $\underline{3}$ (1962) 1008-1011.

86. Hale, J.K., "Oscillations in Non-Linear Systems", McGraw-Hill, New York 1963.

87. Hale, J.K., "Ordinary Differential Equations", Wiley, New York 1969.

88. Robbins, K.A., A New Approach to Subcritical Instability and Turbulent Transitions in a Simple Dynamo, Math. Proc. Cambridge Soc., 82 (1977) 309-325.

89. Haken, H., Analogy Between Higher Instabilities in Fluids and Lasers, Phys: Lett., 53A (1975) 77-78.

90. Lorenz, E.N., Deterministic Non Periodic Flow, J. of the Atmos. Sci., 20. (1963) $130-141$.

91. Dupree, T.H., Theory of Two-Dimensional Turbulence, Phys. of Fluids, 17 (1974) 100-109. 
92. Montgomery, . D., Implications of Navier-Stokes Turbulence Theory for Plasma Turbulence, Proc. Indian Acad. Sci., Sect. A, Vol. 86, 1977, 87-110.

93. Marsden, J.E. and M. MCCracken, "The Hopf Bifurcation and Its Applications," Springer-Verlag, New York 1976.

94. Landau, L.D., C.R. Acad. Sci. URSS, 44 (1944) 311.

95. Landau, L.D. and E.M. Lifschitz,. "Fluid Mechanics", Pergamon Press, oxford 1959.

96. Hopf, E., Abzweigung einer periodischen Lösung von einer stationären Lösung eines Differentialsystems, Berichten der Math.- Phys. Klasse der Sächs. Akad. Wiss: Leipzig. XCIV Band Sitzung vom 19. Jan. 1942. (An English translation can be found in 93).

97. Hopf, E., A Mathematical Example Displaying Features of Turbulence, - Comm. Pure Appl. Math., I (1948) 303-322.

98. Marsden; J., Attempts to Relate the Navier-stokes Equations to Turbulence, in Lect. . Notes in Math \#615, Springer-Verlag, New York 1977.

99. Ruelle; D., The Lorenz Attractor and the Problem of Turbulence, in "Turbulence and Navier-Stokes Equations"; Orsay 1975, Lect. Notes in Math. \#565, Springer-Verlag, New York 1976, p. 146.

100. Ruelle, D. and F. Takens, on the Nature of Turbulence, Comm. Math. Phys., 20. (1971) 167-192; Note concerning our paper "On the Nature of Turbulence", ibid. , 23 (1971) 343-344.

101. Hirsch, M.W. and S. Smale, "Differential Equations, Dynamical Systems, and Linear Algebra", Academic Press, New York 1974.

102. Lanford III, O.E., Qualitative and Statistical Theory of Dissipative Systems, revised text of a series of lectures delivered at the 1976 CIME School of Statistical Mechanics (preprint).

103. Smale, S., Dynamical Systems and Turbulence, in "Turbulence Theory", P. Bernard and T. Ratiu eds, Lect. Notes in Math. \#615, Springer-Verlag, New York 1977.

104. Chenciner, A. and G. Iooss, Bifurcation d'un tore $T^{2}$ en un tore $T^{3}, C \cdot R$. Acad. Sci. Paris, série A, 284 (1977) 1207-1210.

105. Kaplan, J.L. and J.A. Yorke, Preturbulence: a Regime observed in a Fluid Flow Model of Lorenz, preprint March 1977.

106. Gollub, J.P. and H.I. Swinney, Onset of Turbulence in a Rotating Fluid, Phys. Rev, Lett., 35 (1975) 927-930.

107. Swinney, H.L., P.R. Fenstermacher and J.P. Gollub, paper presented at the Symposium on Turbulent Shear Flows, April 18-20, 1977.

$$
-102-
$$


108. Sherman, J. and J. McLaughlin, Power spectra of Nonlinearly Coupled Waves, preprint.

109. MCLaughlin, J.B. and P.C. Martin, Transition to Turbulence in a Statically Stressed Fluid System; Phys: Rev. A, 12 (1975) 186-203.

110. MCLaughlin,.J.B., Successive Bifurcations Leading to stochastic Behavior, J. Stat. Phys., 15 (1976) 307-326.

111. Williams, R.F.; The Structure of the Lorenz Attractors, in "Turbulence Theory", P. Bernard and T. Ratiu eds., Lect. Notes in Math. \#615, SpringerVerlag, New York 1977.

112. Afraimovitch, V.S., V.V. Bikov, and L.P. Shil'nikov, The origin and structure of the Lorenz Attractor, Dokl. Akad. Nauk USSR, 234, n०2 (1977) 336-339; English Trans.: Sov. Phys. Doki., 22, 1977, 253-255.

113. Lorenz, E.N,, Maximum Simplification of the Dynamic Equations, Tellus, 12 (1960) 234-254.

114.. Creveling, H.F., J.F. DePaz, J.V. Balladi, and R.J. Shoenhals, Stability Characteristics of a Single Phase Free Convection Loop, J. Fluid Mech., 67 (197.5) 6.5-84.

115. Kantorovitch, L.V., Functional Analysis and Applied Mathematics, Uspekhi Mat. Nauk, $\underline{3}, \mathrm{n}^{\circ} 6$ (1948) 89-185; English translation: NBS Report 1509, Washington, DC 1952 .

116. Feagin, T. and R.G. Gottlieb; Generalization of Lagrange's Implicit Function Theorem to $n$ Dimensions, Celest: Mech.; 3 (1971) 227-231.

117.. Howland, Jr., R.A., An Accelerated Elimination Technique for the Solution of Perturbed Hamiltonian Systems, Celest. Mech., 15 (1977) 327-352.

118. Bogoliubov, N.N., On Quasi-Periodic Solutions in Nonlinear Problems of Mechanics, First Math. Summer School, Kanev, 1963, Naukova Dumka, Kiev 1964, Vol. 1, p. 11 (in Russian).

119. Rogoliuhov, N.N., Jy. A. Mitropolsky, and A.M. Samoilenko, The Method of Rapid Convergence in Nonlinear Mechanics, Naukova Dumka, Kiev 1969 (in Russian).

120. Cary, J.R., Hamiltonian Perturbation Theory Using Lie Transforms, LBL -635 .

121. The Mathlab Group, MACSYMA Reference Manual, Massachusetts Institute of Technology, 1977.

122. MCNamara, B., Super-convergent Adiabatic Invariants with Resonant Denominators by Lie Transforms, preprint UCRL-79843 Rev. 1, December 2, 1977, to be submitted to J. Math. Phys.. 
123. Hale, J.K., private communication.

124: Hahn, W., Stability of Motion, Springer-Verlag, New York 1967.

125. Bowen, R., A Horseshoe with a Positive Measure, Inventiones Math., 29 (1975) 203-204. 